\title{
Rational equivariant cohomology theories with toral support
}

\author{
J P C GREENLEES
}

\begin{abstract}
For an arbitrary compact Lie group $G$, we describe a model for rational $G$-spectra with toral geometric isotropy and show that there is a convergent Adams spectral sequence based on it. The contribution from geometric isotropy at a subgroup $K$ of the maximal torus of $G$ is captured by a module over $H^{*}\left(B W_{G}^{e}(K)\right)$ with an action of $\pi_{0}\left(W_{G}(K)\right)$, where $W_{G}^{e}(K)$ is the identity component of $W_{G}(K)=N_{G}(K) / K$.
\end{abstract}

55N91, 55P42, 55P91; 55P92, 55T15

\section{Introduction}

\section{A Main result}

For any compact Lie group $G$, rational $G$-equivariant cohomology theories are represented by rational $G$-spectra. Furthermore, the category of $G$-equivariant cohomology theories is the homotopy category of the category of rational $G$-spectra. This category breaks up into mutually orthogonal parts, the most important of which is the toral part: the cohomology theories are those with toral support, and the $G$-spectra are those whose geometric isotropy is a set of subgroups of the maximal torus $\mathbb{T}$.

In this paper we provide an effective method for calculating with toral $G$-spectra. More precisely, we construct an abelian category $\mathcal{A}(G$, toral) of injective dimension equal to the rank of $G$ and a homology functor

$$
\pi_{*}^{\mathcal{A}(G)}: G \text {-spectra } \longrightarrow \mathcal{A}(G, \text { toral }),
$$

so that (Theorem 12.1) there is an Adams spectral sequence

$$
\operatorname{Ext}_{\mathcal{A}(G, \text { toral })}^{*, *}\left(\pi_{*}^{\mathcal{A}(G)}(X), \pi_{*}^{\mathcal{A}(G)}(Y)\right) \Rightarrow[X, Y]_{*}^{G}
$$

convergent for arbitrary rational toral $G$-spectra $X$ and $Y$. The special cases when $G$ is a torus, $O(2)$ and $\mathrm{SO}(3)$ follow from earlier work (see Greenlees $[8 ; 7 ; 9]$ ).

In all cases, the model is assembled from data at individual subgroups $K$. The contribution from $K$ comes from the geometric $K$-fixed point spectrum; this spectrum has an action of the Weyl group $W_{G}(K)=N_{G}(K) / K$, with identity component $W_{G}^{e}(K)$ and 
discrete quotient $W_{G}^{d}(K)=\pi_{0}\left(W_{G}(K)\right)$. When the spectrum is finite the piece of data amounts to taking the $W_{G}^{e}(K)$-equivariant Borel cohomology of its dual and viewing it as a module over $H^{*}\left(B W_{G}^{e}(K)\right.$ ) with an action of $W_{G}^{d}(K)$ (see Proposition 11.5 for a complete statement).

\section{B Background}

If $G$ is a compact Lie group, the author has conjectured (see Greenlees [10]) that one may describe the homotopy theory of rational $G$-spectra in algebraic terms. There are now a good number of examples where this has been proved, including finite groups, tori (see Greenlees and Shipley [15]), $O(2)$ (see Barnes [2; 1; 3]) and $\mathrm{SO}(3)$ (see Kedziorek [17]). The results show that there is a Quillen equivalence between the category of rational $G$-spectra and differential graded objects in a certain abelian category $\mathcal{A}(G)$.

The category $\mathcal{A}(G)$ takes the form of a category of sheaves of modules over a sheaf of rings on the space of closed subgroups of $G$ : the stalk over a subgroup $H$ captures the geometric isotropy information at $H$. Many of the most interesting cohomology theories (including $K$-theory and elliptic cohomology) have the property that the geometric isotropy comes entirely from subgroups of the maximal torus. For example, it is apparent from the groups $O(2)$ and $\mathrm{SO}(3)$ that the part of the model corresponding to isotropy in the maximal torus $\mathbb{T}$ is the most significant and interesting part. The present paper is about this toral part for an arbitrary compact Lie group $G$.

To be more precise, the endomorphism ring of the rational sphere spectrum (the rational Burnside ring $A(G)$ ) acts on the category of $G$-spectra, and in fact it consists of the equivariant sections of the constant sheaf $\mathbb{Q}$ over the space $\mathcal{F} G$ of subgroups of finite index in their normalizer. This means that $A(G)=C_{G}(\mathcal{F} G, \mathbb{Q})$ is the ring of equivariant continuous functions, where $\mathcal{F} G$ has the Hausdorff metric topology. Any open, closed, $G$-invariant subset $S$ of $\mathcal{F} G$ specifies an idempotent $e_{S} \in A(G)$, and the category of rational $G$-spectra and $\mathcal{A}(G)$ both split into two pieces corresponding to the decomposition $1=e_{S}+e_{S^{c}}$. The part corresponding to $e_{S}$ consists of spectra whose geometric isotropy consists of subgroups $L$ cotoral in elements of $S$ (ie $L$ is normal in a subgroup $K$ in $S$ with $K / L$ a torus). In particular, we may take $S$ to consist of the single conjugacy class $(\mathbb{T})$ of maximal tori, and consider the category

$$
\text { toral- } G-\text { spectra }:=e_{(\mathbb{T})}[G-\text { spectra } / \mathbb{Q}]
$$

consisting of $G$-spectra whose geometric isotropy lies inside a maximal torus.

In general we may break up the category of rational $G$-spectra by choosing a finite orthogonal decomposition of the unit of $A(G)$. When $G$ is a torus, $\mathcal{A}(G)$ is indecomposable and $\mathcal{A}(G)=\mathcal{A}(G$, toral). For $G=O(2)$ the category breaks up into the 
toral (or cyclic) part $\mathcal{A}(O(2)$, toral $)=\mathcal{A}(\mathrm{SO}(2))[W]$ as described here, and a piece corresponding to dihedral groups which is simply a graded equivariant sheaf over a compact totally disconnected space with $O(2)$ as an accumulation point (see Greenlees [7] and Barnes [2;1;3]). For $G=\mathrm{SO}(3)$ the category again breaks up into the toral (or cyclic) part $\mathcal{A}(\mathrm{SO}(3)$, toral) as described here, and a piece which is simply a graded equivariant sheaf (see Greenlees [9] and Kedziorek [17]); the graded sheaf piece also breaks up into a piece corresponding to dihedral groups (of order 4 or more) which have $O(2)$ as an accumulation point, and a piece for a number of isolated exceptional subgroups (tetrahedral, octahedral and icosahedral). One should not expect that the nontoral part is always a plain graded sheaf; for example, if $G$ is the product of a circle $T$ and a group of order $2, \mathcal{A}(G)$ splits into the toral part $\mathcal{A}(G$, toral) (as here) and a second part (corresponding to subgroups not inside the maximal torus) which is similar in character to $\mathcal{A}(T)$. The category of toral chains described in Greenlees [6] gives an indication of the expected pattern in general.

\section{C Restriction to the maximal torus}

In a pattern familiar from elsewhere in the theory of transformation groups, we will prove that restricting from $G$-spectra to $\mathbb{T}$-spectra is faithful on the homotopy category of toral $G$-spectra provided we remember the action of the Weyl group $\mathbb{W} G$. This suggests that the putative algebraic model $\mathcal{A}(G$, toral $)=e_{(\mathbb{T})} \mathcal{A}(G)$ for toral $G$-spectra could be described in terms of the category $\mathcal{A}(\mathbb{T})$ defined in Greenlees [11]. This idea will lead us to the construction of a category $\mathcal{A}(G$, toral $)$ and a homology theory on $G$-spectra with values in $\mathcal{A}(G$, toral). We will show that this is a good invariant in the sense that it is calculable and gives a convergent Adams spectral sequence for maps between toral spectra.

Consideration of the torus-normalizer $\mathbb{N}=N_{G}(\mathbb{T})$ is central to the analysis. The general theory simplifies for $\mathbb{N}$, since the identity component is itself the maximal torus, and we find $\mathcal{A}(\mathbb{N}$, toral $)=\mathcal{A}(\mathbb{T})[\mathbb{W} G]$ (the category of $\mathbb{W} G$-equivariant objects of $\mathcal{A}(\mathbb{T})$ in a sense made precise below). We show that restriction from $G$-spectra to $\mathbb{N}$-spectra is full and faithful on homotopy categories. In summary, we construct a diagram

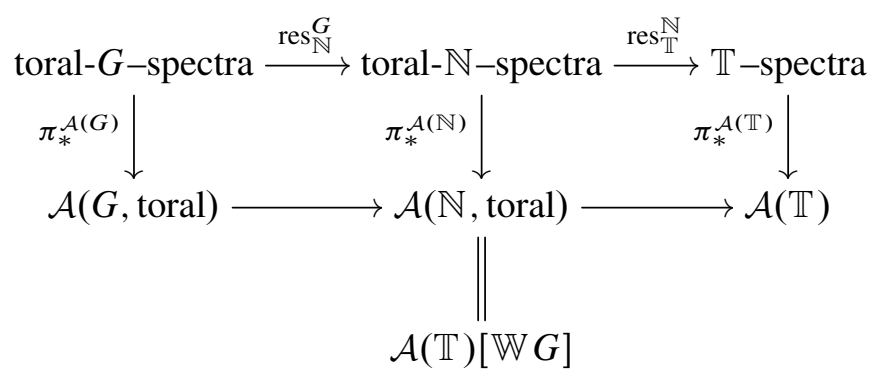


with convergent Adams spectral sequences based on each of the vertical homology functors. Taken together with the examples mentioned above, this is strong evidence for the conjecture that the category of toral $G$-spectra is Quillen equivalent to the category of differential graded objects in $\mathcal{A}(G$, toral).

\section{D The form of the model of toral $G$-spectra}

As suggested by known examples, we expect the stalk of $\mathcal{A}(G$, toral) over a subgroup $K$ to be a module over $H^{*}\left(B W_{G}^{e} K\right)$, where $W_{G}^{e}(K)$ is the identity component of the Weyl group $W_{G} K=N_{G} K / K$, and we expect an action of the discrete quotient $W_{G}^{d} K=\pi_{0}\left(W_{G} K\right)$. More precisely, we expect a module over the twisted group ring

$$
\mathbb{R}_{\mathrm{tw}}^{G}(K)=H^{*}\left(B W_{G}^{e} K\right)\left[W_{G}^{d} K\right] .
$$

Understanding the restriction from $G$-spectra to $\mathbb{N}$-spectra involves some rather interesting pieces of invariant theory.

The relationship between the stalks is given by the localization theorem for cotoral inclusions. If $G=\mathbb{T}$ is a torus and $L$ is cotoral in $K$ then we have a group homomorphism $W_{G} L=\mathbb{T} / L \rightarrow \mathbb{T} / K=W_{G} K$ which forms the basis of this. For a general group $G$ we cannot expect a map $N_{G} L \rightarrow N_{G} K$ (consider $L=1$ ), so we think of rings and modules associated to cotoral flags of subgroups, and this restores functoriality. We recall how this works for tori in the next subsection.

\section{$1 \mathrm{E}$ The model for $\mathbb{T}$-spectra}

We sketch the construction of the model $\mathcal{A}(\mathbb{T})$ for rational $\mathbb{T}$-spectra, referring to Greenlees [14] for fuller details (the model described here is the $(a, f)$-model, based on flags involving all closed subgroups). The starting point of the discussion is the poset $\Sigma_{a}$ consisting of all closed subgroups. The partial order is given by cotoral inclusion, so that $K \supseteq L$ if $L$ is a subgroup of $K$ and $K / L$ is a torus. We then consider the poset flag $\left(\Sigma_{a}\right)$ consisting of flags $\left(K_{0} \supset K_{1} \supset \cdots \supset K_{s}\right)$ in $\Sigma_{a}$. We may define a $\Sigma_{a}$-diagram $\mathbb{R}_{a}$ of rings by

$$
\mathbb{R}_{a}(K):=H^{*}(B \mathbb{T} / K) .
$$

If $K \supseteq L$ then the projection $\mathbb{T} / K \leftarrow \mathbb{T} / L$ induces the inflation map $\mathbb{R}_{a}(K) \rightarrow \mathbb{R}_{a}(L)$, making $\mathbb{R}_{a}$ into a contravariant functor on $\Sigma_{a}$.

Using Euler classes, we may form a flag $\left(\Sigma_{a}\right)$-diagram of rings. Since the values on flags of length 0 agree with those of $\mathbb{R}_{a}$, we continue to use $\mathbb{R}_{a}$ for the functor on flags. First, if $K \supseteq L$ we may consider the set

$$
\mathcal{E}_{K / L}:=\left\{e(W) \mid W \in \operatorname{Rep}(\mathbb{T} / L), W^{K}=0\right\}
$$


of Euler classes of $K$-essential representations of $\mathbb{T} / L$. Here $e(W) \in H^{|W|}(B \mathbb{T} / L)$ is the Euler class of $W$. Now we may define the flag diagram $\mathbb{R}_{a}$ by

$$
\mathbb{R}_{a}\left(K_{0} \supset K_{1} \supset \cdots \supset K_{S}\right):=\mathcal{E}_{K_{0} / K_{S}}^{-1} H^{*}\left(B \mathbb{T} / K_{S}\right) .
$$

We note that this only depends on the first and last term in the flag and it is a covariant functor on $\operatorname{flag}\left(\Sigma_{a}\right)$. It is also important to note that if $K \supset L$ the values at $K$ and $L$ are not linked directly in flag $\left(\Sigma_{a}\right)$, but rather through the zigzag induced by the inclusions $(K) \rightarrow(K \supset L) \leftarrow(L)$.

The category $\mathcal{A}(\mathbb{T})$ is a category of modules $M$ over the flag $\left(\Sigma_{a}\right)$-diagram $\mathbb{R}_{a}$ : thus $M$ is a diagram of abelian groups so that if $E \subseteq F$, the map $M(E) \rightarrow M(F)$ is a map of modules over $\mathbb{R}_{a}(E) \rightarrow \mathbb{R}_{a}(F)$. The modules in $\mathcal{A}(\mathbb{T})$ are required to be quasicoherent $(q c)$, extended $(e)$ and $\mathcal{F}$-continuous. A module is quasicoherent if the value is determined by the last term in the flag by extensions of scalars, that is, if $F=\left(K_{0} \supset K_{1} \supset \cdots \supset K_{S}\right)$ then the inclusion $\left(K_{S}\right) \rightarrow F$ induces an isomorphism

$$
M(F)=\mathbb{R}_{a}(F) \otimes_{\mathbb{R}_{a}\left(K_{s}\right)} M\left(K_{s}\right) .
$$

A module is extended if the value is determined by the first term in the flag by extensions of scalars, that is, if $F=\left(K_{0} \supset K_{1} \supset \cdots \supset K_{S}\right)$ then the inclusion $\left(K_{0}\right) \rightarrow F$ induces an isomorphism

$$
M(F)=\mathbb{R}_{a}(F) \otimes_{\mathbb{R}_{a}\left(K_{0}\right)} M\left(K_{0}\right) .
$$

The $\mathcal{F}$-continuity condition is a finiteness condition described in Section $6 \mathrm{E}$ below.

Since the values of both $\mathbb{R}_{a}$ and $M$ are determined by the first and last term in any flag we will sometimes simplify the notation by giving the value only on cotoral pairs $(K \supset L)$. The point of defining $\mathbb{R}_{a}$ on flags is to give functoriality and control automorphisms.

Our approach to constructing $\mathcal{A}(G$, toral $)$ is to take into account the action of the Weyl group $\mathbb{W} G=N_{G}(\mathbb{T}) / \mathbb{T}$ on the poset $\Sigma_{a}(\mathbb{T})$ of all subgroups of the maximal torus. Indeed, $\mathbb{W} G$ acts on the diagram $\mathbb{R}_{a}$ of polynomial rings, and it turns out that by descent this gives us the model $\mathcal{A}(G$, toral). We develop the appropriate machinery, and finally give the definition in Section $6 \mathrm{~F}$.

\section{$1 F$ Layout of the paper}

The paper is divided into two parts. Part I ("Algebra") develops the algebraic framework and Part II ("Topology") applies it to calculations with $G$-spectra.

In Section 2 we introduce notation from the theory of compact Lie groups and make some elementary observations, and in Section 3 we recall facts about the cohomology of classifying spaces of compact Lie groups. 
We then spend several sections developing machinery to discuss categories of modules over a diagram of rings on which a finite group acts. In Section 4 the categorical setup is described and in Section 5 this is specialized to categories of modules over an equivariant diagram of rings and the fundamental descent adjunction is proved. Finally, Section 6 specializes to the example arising from compact Lie groups, and gives the definition of $\mathcal{A}(G$, toral $)$. The fact that the descent adjunction respects quasicoherent extended modules contains information on which $\mathbb{N}$-equivariant objects are restrictions of $G$-equivariant objects; this is surprisingly subtle and treated in Section 7. As a first step towards homotopy theory, we then consider the homological algebra of $\mathcal{A}(G$, toral), identifying enough injectives and showing its injective dimension is equal to the rank of $G$.

We then turn to topology. The fundamental result proved in Section 9 is that toral phenomena are detected on restriction to the maximal torus. In preparation for work on the Adams spectral sequence we then reformulate some well-known properties of Borel cohomology in Section 10; this is the route by which the classical theory of characteristic classes of principal bundles enters the model.

Section 11 explains the relationship between $\mathcal{A}(\mathbb{T})$ and $\mathcal{A}(G$, toral $)$ and thereby allows us to construct the functor $\pi_{*}^{\mathcal{A}(G)}$ from $G$-spectra to $\mathcal{A}(G$, toral $)$. This is then used in Section 12 to construct the Adams spectral sequence, with the hard work deferred to Section 13 where enough injectives are realized, and Section 14 where it is shown that maps into the resulting spectra are detected in $\mathcal{A}(G$, toral $)$. The Adams spectral sequence lets one calculate maps, and this is complemented in Section 15 by a proof that the functor $\pi_{*}^{\mathcal{A}}$ is essentially surjective: all objects of $\mathcal{A}(G$, toral) do occur as $\pi_{*}^{\mathcal{A}(G)}(X)$ for a toral $G$-spectrum $X$. Finally, Section 16 explains how restriction, induction and coinduction are reflected at the level of algebraic models.

\section{$1 G$ Conventions}

All groups will be compact Lie groups, and if connectedness is required this will be stated explicitly. All subgroups will be required to be closed. Generally, containment of subgroups follows the alphabet, as in $G \supseteq H$.

Cohomology is unreduced unless indicated, and always has rational coefficients.

Acknowledgement I am grateful to MSRI for support and providing an excellent environment for organizing these ideas during the algebraic topology programme in 2014. 


\section{Part I Algebra}

\section{Weyl groups}

The algebraic input to our results is the classical structure and representation theory of compact Lie groups. Although this is well known, the recollection of standard facts gives an opportunity to introduce notation. Readers have found the list of standard notation in Section $2 \mathrm{C}$ valuable. We recommend consideration of the rotation group $G=\operatorname{SO}(3)$ as an example to illustrate results.

\section{A Two types of Weyl groups}

For a compact Lie group $G$ we write $G_{e}$ for its identity component and we write $G_{d}=G / G_{e}=\pi_{0}(G)$ for the discrete quotient. A closed subgroup $K$ of $G$ has normalizer $N K=N_{G} K$, and Weyl group $W K=W_{G} K=N_{G} K / K$. More generally given a flag $F=\left(K_{0} \supset K_{1} \supset \cdots \supset K_{S}\right)$ of subgroups of $G$ we write

$$
N_{G}(F)=N_{G}\left(K_{0}\right) \cap \cdots \cap N_{G}\left(K_{s}\right)
$$

for the subgroup normalizing all terms in the flag and

$$
W_{G}(F)=N_{G}(F) / K_{S}
$$

for its Weyl group.

Moving on to the theory of compact Lie groups, we write $\mathbb{T}=\mathbb{T} G$ for the maximal torus of $G$ and $\mathbb{N}=\mathbb{N} G=N_{G}(\mathbb{T})$ for its normalizer. The Weyl group $W_{G}(\mathbb{T})=N_{G}(\mathbb{T}) / \mathbb{T}$ of the maximal torus is a finite group that plays a central role in the theory, so we use the notation

$$
\mathbb{W} G=W_{G} \mathbb{T} .
$$

Since $W_{G}(G)=1$, there is little danger of confusion as long as the reader bears in mind there are two meanings of the phrase "Weyl group".

For most of this paper we will suppose $K$ is a subgroup of $\mathbb{T}$, so that $\mathbb{T} \subseteq N_{G} K$.

\section{B Weyl groups of Weyl groups}

We will need to consider $W K$ as a Lie group in its own right, with maximal torus $\mathbb{T} W K$ and Weyl group $\mathbb{W} W K=W_{W K}(\mathbb{T} W K)=N_{W K}(\mathbb{T} W K) / \mathbb{T} W K$. We may simplify this notation slightly.

Lemma 2.1 The maximal torus of $W K$ is given by $\mathbb{T} W K=\mathbb{T} / K$. 
Proof Certainly $\mathbb{T} / K$ is a torus in $W K$. If there were a bigger one it would have the form $T^{\prime} / K$ for some subgroup $T^{\prime}$ of $N K$ containing $\mathbb{T}$. Then we would have a chain $T^{\prime} \supseteq \mathbb{T} \supseteq K$. The group $T^{\prime} / \mathbb{T}$ is a quotient of the torus $T^{\prime} / K$ and hence is itself a torus. Thus $T^{\prime}$ is itself a torus; by maximality of $\mathbb{T}$ we have $\mathbb{T}=T^{\prime}$.

Lemma 2.2 The normalizer of the maximal torus of $W K$ is given by

$$
N_{W K}(\mathbb{T} W K)=N_{W K}(\mathbb{T} / K)=N_{G}(\mathbb{T} \supseteq K) / K=\left(N_{G} \mathbb{T} \cap N_{G} K\right) / K .
$$

It follows that the toral Weyl group of $W_{G} K$,

$$
\mathbb{W} W_{G} K=\left(N_{G} \mathbb{T} \cap N_{G} K\right) / \mathbb{T} \subseteq N_{G} \mathbb{T} / \mathbb{T}=\mathbb{W} G,
$$

is the subgroup of $\mathbb{W} G$ normalizing $K$. With the usual notation for the isotropy group of the action of $\mathbb{W} G$ on the set of subgroups of $\mathbb{T}$ we have

$$
\mathbb{W}\left(W_{G} K\right)=(\mathbb{W} G)_{K}
$$

Proof The first equality is the previous lemma. Now any element $g$ of $G$ normalizing $\mathbb{T} \supseteq K$ is in $N K$ and hence defines an element $g K$ of $W K$. We then have $(g K)(t K)(g K)^{-1}=g t g^{-1} K$. This is in $\mathbb{T}$ by hypothesis, and hence we have a homomorphism

$$
N_{G}(\mathbb{T} \supseteq K) \longrightarrow N_{W K}(\mathbb{T} / K) .
$$

Evidently $K$ is in the kernel, and since $N_{W K}(\mathbb{T} / K) \subseteq W K$ we have a monomorphism

$$
N_{G}(\mathbb{T} \supseteq K) / K \longrightarrow N_{W K}(\mathbb{T} / K) .
$$

Now suppose $g K \in W K$ normalizes $\mathbb{T} / K$, which is to say that for any $t \in \mathbb{T}$,

$$
\mathbb{T} / K \ni(g K)(t K)(g K)^{-1}=g t g^{-1} K .
$$

It follows that $\operatorname{gtg}^{-1} \in \mathbb{T}$ and $g$ normalizes $\mathbb{T}$.

\section{C Summary of notation}

Associated to a subgroup $K$ of $\mathbb{T}$ we have

- $G$, the ambient compact Lie group,

- $G_{e}$, the identity component of $G$,

- $G_{d}=G / G_{e}=\pi_{0}(G)$, the group of components of $G$,

- $\mathbb{T}=\mathbb{T} G$, the maximal torus of $G$,

- $\mathbb{N}=\mathbb{N} G=N_{G}(\mathbb{T})$, 
- $\mathbb{W}=\mathbb{W} G=\mathbb{N} G / \mathbb{T} G$, the toral Weyl group of $G$,

- $K$, a closed subgroup of $\mathbb{T} G$,

- $N K=N_{G} K$,

- $W K=W_{G} K$, with identity component $W^{e} K=W_{G}^{e} K=\left(W_{G} K\right)_{e}$ and component group $W^{d} K=W_{G}^{d} K=\left(W_{G} K\right)_{d}$,

- $\mathbb{T} W K=\mathbb{T} / K$,

- $\mathbb{N} W K=N_{W K}(\mathbb{T} W K)=(N \mathbb{T} \cap N K) / K$,

- $\mathbb{W} W K=(N \mathbb{T} \cap N K) / \mathbb{T}=(\mathbb{W} G)_{K}$ acting on $\mathbb{T} W K=\mathbb{T} / K$.

\section{Cohomology of classifying spaces of compact Lie groups}

The relationship between the rational cohomology of classifying spaces of $G, \mathbb{N}$ and $\mathbb{T}$ proved by Borel is fundamental to our entire analysis.

\section{A Cohomology of classifying spaces and free spectra}

Borel's calculation of the rational cohomology of classifying spaces is as follows.

Lemma 3.1 (Borel) For a compact Lie group $G$ with maximal torus $\mathbb{T}, \mathbb{N}=N_{G}(\mathbb{T})$ and $\mathbb{W}=N_{G}(\mathbb{T}) / \mathbb{T}$ we have

$$
H^{*}(B G)=H^{*}(B \mathbb{N})=H^{*}(B \mathbb{T})^{\mathbb{W}} .
$$

We may apply this to Weyl groups to see

$$
H^{*}(B W K)=H^{*}(B \mathbb{N} W K)=H^{*}(B \mathbb{T} W K)^{\mathbb{W} W K}=H^{*}(B \mathbb{T} / K)^{\mathbb{W} W K} .
$$

Cohomology of classifying spaces plays a fundamental role in equivariant stable homotopy theory.

Theorem 3.2 (Greenlees and Shipley [16]) The category of free rational $W_{G} K-$ spectra is Quillen equivalent to the category of torsion modules over the twisted group ring

$$
H^{*}\left(B W_{G}^{e} K\right)\left[W_{G}^{d} K\right]=H^{*}(B \mathbb{T} / K)^{\mathbb{W} W_{G}^{e} K}\left[W_{G}^{d} K\right]
$$

This embodies the role of the cohomology of classifying spaces in modelling rational stable equivariant homotopy theory. 


\section{B Identity components, 1}

The maximal torus only depends on the identity component of a group, so $\mathbb{T}\left(G_{e}\right)=\mathbb{T} G$.

Lemma 3.3 There are short exact sequences

$$
\begin{gathered}
1 \longrightarrow N_{G_{e}} \mathbb{T} \longrightarrow N_{G} \mathbb{T} \longrightarrow G_{d} \longrightarrow 1, \\
1 \longrightarrow \mathbb{W} G_{e} \longrightarrow \mathbb{W} G \longrightarrow G_{d} \longrightarrow 1 .
\end{gathered}
$$

Proof $G_{e}$ acts transitively on maximal tori. So for any $\gamma \in G_{d}$ represented by $\tilde{\gamma} \in G$ there is an $x \in G_{e}$ with $\mathbb{T}=\left(\mathbb{T}^{\tilde{\gamma}}\right)^{x}$ and $\tilde{\gamma} x \in N_{G}(\mathbb{T})$. Since $N_{G_{e}}(\mathbb{T})=N_{G}(\mathbb{T}) \cap G_{e}$, this gives the exact sequence.

This fits well with the following picture:

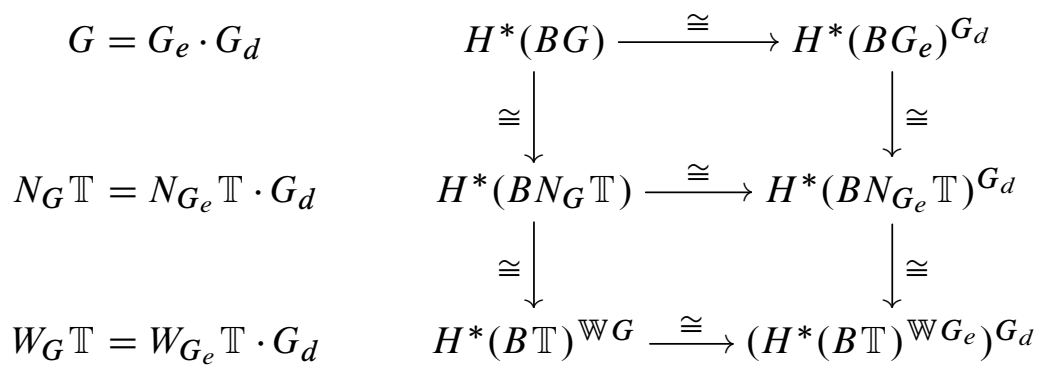

\section{C Identity components, 2}

We note that $\mathbb{N}$ acts on the set of subgroups of $\mathbb{T}$ by conjugation, and that this passes to an action of $\mathbb{W}$. Recall that

$$
\mathbb{W} W_{G} K=\left(N_{G} K \cap \mathbb{N}\right) / \mathbb{T}=(\mathbb{W} G)_{K}
$$

Lemma 3.4 There is a short exact sequence

$$
1 \longrightarrow \mathbb{W}\left(W_{G}^{e} K\right) \longrightarrow \mathbb{W}\left(W_{G} K\right) \longrightarrow W_{G}^{d} K \longrightarrow 1 .
$$

Under the identification $\mathbb{W}\left(W_{G} K\right)=(\mathbb{W} G)_{K}$, the subgroup $\mathbb{W}\left(W_{G}^{e} K\right)$ corresponds to the set of elements of the toral Weyl group $\mathbb{W} G$ represented by the identity component of $N_{G}(K)$ :

$$
\left(K \cdot\left(N_{G} K\right)_{e} \cap \mathbb{N}\right) / \mathbb{T}=\mathbb{W}\left(W_{G}^{e} K\right) .
$$

Remark 3.5 The Weyl group $\mathbb{W} W_{G}^{e} K$ can be very small or very large. At one extreme, if $K=\mathbb{T}$ it is trivial. At the other, if $K=1$ then $N_{G}(K)=G$ and if $G$ is connected we obtain the entire Weyl group $\mathbb{W} G$.

Proof The short exact sequence is obtained by applying Lemma 3.3 to $W K$. Now note $\mathbb{W}\left(W_{G}^{e} K\right)=N_{W_{G}^{e} K}(\mathbb{T} / K) /(\mathbb{T} / K)$ and $W_{G}^{e} K=K \cdot\left(N_{G} K\right)_{e} / K$. 


\section{Equivariant diagrams}

We are going to discuss diagrams of rings and modules with group action. The basic examples arise from the algebraic models of rational $\mathbb{T}$-spectra [14], recalled briefly in Section 1E. The diagram shapes $\Sigma$ come from the set $\Sigma_{a}(\mathbb{T})$ of all closed subgroups of $\mathbb{T}$ under cotoral inclusion (in the present context, simply inclusions with connected quotient). One such poset $\Sigma$ is $\Sigma_{a}$ itself, but we also need to consider the poset flag $\left(\Sigma_{a}\right)$ of flags in $\Sigma_{a}$. Accordingly, we discuss the relevant structures with $\Sigma$ unspecified, which has the added benefit of clarifying the structure.

\section{A Diagrams with an action}

We need to consider the general setup of a group $W$ acting on the right of a poset $\Sigma$. We want a notion of equivariant $\Sigma$-diagrams in a category $\mathbb{C}$. We start by considering the functor category $\mathbb{C}^{\Sigma}$. This admits an action of $W$, where the image of a functor $F: \Sigma \rightarrow \mathbb{C}$ under $w \in W$ is the functor $w_{*} F$ defined by

$$
\left(w_{*} F\right)(\sigma):=F\left(\sigma^{w}\right) .
$$

One quickly verifies $v_{*} w_{*} F=(v w)_{*} F$ and $e_{*} F=F$.

An equivariant diagram is then a diagram $F$ with additional structure. We specify an action by $W$ on $F$ by giving maps

$$
w_{m}: F \longrightarrow w_{*} F
$$

with $e_{m}=1$ and $v_{m} w_{m}=(v w)_{m}$. It is more flexible to give an alternative point of view in which an equivariant $\Sigma$-diagram is just a diagram of a more complicated shape.

\section{B Orbifold posets}

We want to consider a class of categories $A$ that are based on a poset $\Sigma$, but with automorphisms added.

Definition 4.1 A $\Sigma$-orbifold is a category $A$ with the same objects as $\Sigma$ equipped with functors $\Sigma \rightarrow A \rightarrow \Sigma$ which are the identity on objects so that

(1) $A$ has finitely many morphisms between any two objects,

(2) the morphisms of $A$ are generated by those of $\Sigma$ together with the automorphisms, and

(3) every endomorphism in $A$ is an isomorphism.

The trivial $\Sigma$-orbifold associated to the finite group $W$ is $A=\Sigma \times W$ with structure maps coming from $1 \rightarrow W \rightarrow 1$. 


\section{C The transport category}

Starting with a poset $\Sigma$ with a right action of a finite group $W$, we may form the transport category $\Sigma \rtimes W$. This is a $\Sigma$-orbifold with morphism set $\Sigma \times W$ and structure maps induced by $1 \rightarrow W \rightarrow 1$. In giving formulae for composition we are following through the convention that the action of $W$ is on the right, so that functions also operate on the right.

If $i: \sigma \rightarrow \tau$ and $v \in W$ the morphism $(i, v)$ has domain $\sigma$ and codomain $\tau^{v}$. The composite of $(i, v)$ and $(j, w)$ where $j: \tau^{v} \rightarrow \phi^{v}$ is given by the formula

$$
(i, v)(j, w)=\left(i j^{v^{-1}}, v w\right) .
$$

Note that $(i, v)$ is the composite $\sigma \stackrel{i}{\longrightarrow} \tau \stackrel{v}{\longrightarrow} \tau^{v}$. Since $W$ acts on $\Sigma$ as a poset, we may find a commutative square

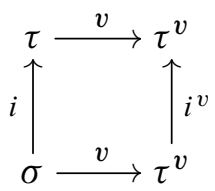

and

$$
i v=(i, v)=v i^{v} \text {. }
$$

In particular the group of self-maps of $\sigma$ as an object of $\Sigma \rtimes W$ is the isotropy group $W_{\sigma}$. It is clear we can repackage the notion of a $W$-equivariant diagram in terms of $\Sigma \rtimes W$.

Lemma 4.2 The category of $W$-equivariant $\Sigma$-diagrams in $\mathbb{C}$ is equivalent to the category of functors $\Sigma \rtimes W \rightarrow \mathbb{C}$.

Proof Equivariant diagrams $\left(F,\left\{w_{m}\right\}_{w \in W}\right)$ are related to functors $F^{\prime}: \Sigma \rtimes W \rightarrow \mathbb{C}$ by taking $F^{\prime}(\sigma)=F(\sigma)$ and $F^{\prime}(w)=w_{m}$.

\section{D Component structures}

The purpose of the formulation in terms of the transport category $\Sigma \rtimes W$ is to let us to capture the behaviour of the identity components of Weyl groups.

Definition 4.3 A component structure on $\Sigma \rtimes W$ is a sub- $\Sigma$-orbifold $W_{\bullet}^{e}$. Given a component structure, the endomorphism object of $\sigma$ is written $W_{\sigma}^{e}$.

A component structure is normal if $W_{\sigma}^{e}$ is normal in $W_{\sigma}$. 
Lemma 4.4 If the component structure is normal, then the discrete residual is the $\Sigma$-orbifold $W_{\bullet}^{d}$ with a sequence of maps

$$
W_{\bullet}^{e} \longrightarrow \Sigma \rtimes W \longrightarrow W_{\bullet}^{d}
$$

of $\Sigma$-orbifolds that is exact in the sense that it defines an isomorphism $W_{\sigma}^{d} \cong W_{\sigma} / W_{\sigma}^{e}$.

Proof The morphisms in $W_{\bullet}^{d}$ are pairs $(i,[v])$ where $i: \sigma \rightarrow \tau$ and were $[v]$ is the equivalence class of $v \in W$ under precomposition by $W_{\tau}^{e}$ and postcomposition by $W_{\tau^{v}}^{e}$. The composition is induced from the composition of $\Sigma \rtimes W$. The normality condition enables one to check that this is well defined.

Example 4.5 (i) For any $W$ we may define the connected component structure by $W_{\sigma}^{e}=W_{\sigma}$ giving $W_{\sigma}^{d}=1$.

(ii) For any $W$ we may define the discrete component structure by $W_{\sigma}^{e}=1$ giving $W_{\sigma}^{d}=W_{\sigma}$.

We devote a separate subsection to the motivating example that will concern us for most of the paper.

\section{E The compact Lie group component structure}

The motivating example comes from a compact Lie group $G$. We take $\Sigma=\Sigma_{a}(\mathbb{T})$ and the Weyl group $W=\mathbb{W} G$ acts by conjugation in the usual way.

The component structure corresponds to the identity components of the Weyl groups

$$
W_{K}^{e}=(\mathbb{W} G)_{K}^{e}=\mathbb{W} W_{G}^{e} K
$$

Accordingly, by Lemma 3.3, the discrete residual is

$$
W_{K}^{d}=(\mathbb{W} G)_{K}^{d}=W_{G}^{d} K
$$

Example 4.6 If the identity component of $G$ is the maximal torus $\mathbb{T}$, so that $G=\mathbb{N}$, we have $W_{G}^{e} K=\mathbb{T} / K$ which has trivial toral Weyl group, and the component structure is the discrete component structure

$$
(\mathbb{W} \mathbb{N})_{K}^{e}=1 \quad \text { and } \quad(\mathbb{W} \mathbb{N})_{K}^{d}=(\mathbb{W} \mathbb{N})_{K}
$$

Example 4.7 If $G=\mathrm{SO}(3)$ we have $\mathbb{T}=\mathrm{SO}(2), \mathbb{N}=O(2)$ and $\mathbb{W}=C_{2}$. The subgroups of $\mathbb{T}$ are the cyclic subgroups $C_{n}$ of finite order $n$, and $\mathbb{T}$ itself. All these subgroups are characteristic and hence

$$
(\mathbb{W} G)_{K}=\mathbb{W} G \text { for all } K \subseteq \mathbb{T} .
$$


The trivial subgroup $C_{1}$ has normalizer $G$ and Weyl group $W_{G}\left(C_{1}\right)=G$. The other subgroups of $\mathbb{T}$ have normalizer $\mathbb{N}$. Thus the finite subgroups have Weyl group isomorphic to $O(2)$ and $\mathbb{T}$ has Weyl group $\mathbb{W} G$. The associated component structure thus has

$$
(\mathbb{W} G)_{K}^{e}= \begin{cases}\mathbb{W} G & \text { if } K=C_{1} \\ 1 & \text { otherwise }\end{cases}
$$

and discrete residual

$$
(\mathbb{W} G)_{K}^{d}= \begin{cases}1 & \text { if } K=C_{1}, \\ \mathbb{W} G & \text { otherwise. }\end{cases}
$$

Example 4.8 The group $G=\mathrm{SU}(3)$ has maximal torus $\mathbb{T}$ of rank 2 consisting of diagonal matrices. There are three (conjugate) subgroups isomorphic to SU(2) which fix the first, second or third complex coordinate. The Weyl group is the symmetric group of degree 3 generated by the three corresponding reflections.

We have

$$
1=(\mathbb{W} G)_{\mathbb{T}}^{e} \subset(\mathbb{W} G)_{\mathbb{T}}=\mathbb{W} G
$$

For subgroups $K \subset \mathbb{T}$ of dimension 1 , we consider the identity component $K_{e}$. If it is one of the three circles fixed by the three reflections in $\mathbb{W} G$ then the normalizer contains the corresponding $\mathrm{SU}(2)$ and

$$
(\mathbb{W} G)_{K}^{e}=(\mathbb{W} G)_{K}=\mathbb{W} \operatorname{SU}(2)
$$

If $K_{e}$ is another circle then

$$
(\mathbb{W} G)_{K}^{e}=(\mathbb{W} G)_{K}=1 .
$$

If $K=1$ then $W_{G}(K)=G$ and

$$
(\mathbb{W} G)_{K}^{e}=(\mathbb{W} G)_{K}=\mathbb{W} G
$$

This is enough to show the richness of the structure; the individual analysis necessary for the remaining cases can await applications.

\section{Equivariant diagrams of rings and modules}

We now specialize the discussion of Section 4 to the case when $\mathbb{C}$ is the category of commutative rings with a view to establishing the descent adjunction (Proposition 5.9). 


\section{A Equivariant diagrams of rings}

Our aim is to describe a descent theory, relating modules over an equivariant diagram of rings and modules over the fixed points under a component structure. We need to impose a restriction on a component structure for this to make sense.

Definition 5.1 We say that a component structure $W_{\bullet}^{e}$ on the $W$-poset $\Sigma$ is decreasing if $E \supseteq F$ implies $W_{E}^{e} \subseteq W_{F}^{e}$.

Example 5.2 (i) If $G$ is a connected compact Lie group but not a torus then $\Sigma_{a}$ with the Lie group component structure of Section 4E is not decreasing: the subgroup $K=1$ has $W_{G}(1)=G$, with nontrivial Weyl group $W_{1}^{e}=\mathbb{W} G$, whereas the subgroup $K=\mathbb{T}$ has discrete Weyl group $W_{G}(\mathbb{T})$, so that $W_{\mathbb{T}}^{e}=1$.

(ii) If $G$ is any compact Lie group then flag $\left(\Sigma_{a}(\mathbb{T})\right)$ with the Lie group component structure of Section $4 \mathrm{E}$ is decreasing. This is immediate from the fact that

$$
N_{G}\left(K_{0} \supset \cdots \supset K_{s}\right)=N_{G}\left(K_{0}\right) \cap \cdots \cap N_{G}\left(K_{S}\right) .
$$

The fact that flags give a decreasing structure whereas subgroups do not explains why we have changed notation for the objects of our poset.

Lemma 5.3 Given a $W$-equivariant $\Sigma$-diagram of rings with a decreasing component structure $W_{\bullet}^{e}$, the definition

$$
R_{\mathrm{inv}}(F)=R(F)^{W_{F}^{e}}
$$

gives a $\Sigma$-diagram of rings.

If the component structure is normal, $R_{\mathrm{inv}}$ defines a $W_{\bullet}^{d}$-diagram of rings.

Proof A map $i: E \rightarrow F$ induces a map $R(i): R(E) \rightarrow R(F)$ and we need to show this induces a map for $R_{\text {inv }}$, namely

$$
R_{\text {inv }}(E)=R(E)^{W_{E}^{e}} \longrightarrow R(F)^{W_{F}^{e}}=R_{\text {inv }}(F) .
$$

The original map $R(i)$ is equivariant for the inclusion $W_{F}^{e}$. Since the component structure is decreasing, any $W_{E}^{e}$-invariant element of $R(E)$ is $W_{F}^{e}$ invariant, and so maps to a $W_{F}^{e}$-invariant element of $R(F)$, and hence $R_{\text {inv }}(i)$ is the composite of $R(i)^{W_{E}^{e}}$ and inclusion.

To see this induces a map on the entire diagram $W_{\bullet}^{d}$, we need only observe that the original structure maps only depend on double coset representatives. 
An alternative language for describing the resulting structure is that of twisted group rings: if a discrete group $\Gamma$ acts on a ring $R$, the twisted group ring $R[\Gamma]$ has an additive $R$-basis consisting of the group elements $\gamma \in \Gamma$ and multiplication is given by $(r \lambda)(s \gamma)=\left(r s^{\lambda^{-1}}\right)(\lambda \gamma)$.

Lemma 5.4 The $W_{\bullet}^{d}$-diagram $R_{\text {inv }}$ defines the twisted invariant ring

$$
R_{\mathrm{tw}}(K)=R(K)^{W_{K}^{e}}\left[W_{K}^{d}\right],
$$

and this defines a $\Sigma$-diagram of noncommutative rings.

Proof Twisted group rings are defined precisely so that the action of the group $W_{E}^{d}$ of endomorphisms of the object $E$ are reflected in a ring acting on the value at $E$. Since all morphisms are generated by the poset maps and groups of self-isomorphisms, the twisted group rings give the entire structure.

\section{B Equivariant diagrams of modules}

The two formulations of $W$-equivariant diagrams of rings have counterparts for modules.

Definition 5.5 If $R$ is a $W$-equivariant $\Sigma$-diagram of rings, a $W$-equivariant module is an $R$-module which is $W$-equivariant in the sense that $w_{m}(\lambda x)=w_{m}(\lambda) w_{m}(x)$ for $\lambda \in R, x \in M$ and $w \in W$.

Lemma 5.6 The category of $W$-equivariant $R$-modules is equivalent to the category of modules over the corresponding $\Sigma \rtimes W$-diagram of rings.

Proof Both $\Sigma$ and $\Sigma \rtimes W$ have the same object set. The morphism $(i, v): \sigma \rightarrow \tau^{v}$ is a composite of $(i, e)$ and $(1, v)$. The latter morphism corresponds to the structure map $v_{m}: M \rightarrow v_{*} M$. The conditions that the actions on rings and modules are compatible in the two cases correspond to each other.

Passing to coset representatives we obtain the result for $R_{\text {inv }}-$ modules.

Lemma 5.7 The category of $W_{\bullet}^{d}$-diagrams of $R_{\mathrm{inv}}$-modules is equivalent to the category of $\Sigma$-diagrams of modules over $R_{\mathrm{tw}}$.

Proof Since the conditions that the actions on rings and modules are compatible in the two cases correspond to each other, this follows by applying the comparison from Lemma 4.2 to modules. 


\section{C $\quad R$-modules and $R_{\text {inv }}-$ modules}

A basic technique of equivariant topology is to relate modules over tori to modules over general groups by suitable descent theorems. We are now equipped to formulate and prove a fundamental adjunction which provides the abstract basis for reducing from $G$-equivariant data to $\mathbb{T}$-equivariant data.

Suppose we have a decreasing component structure $W_{\bullet}^{e}$, and let $\theta: R_{\text {inv }} \rightarrow R$ be the map of $\Sigma$-diagrams defined by the inclusions $\theta(E): R_{\text {inv }}(E)=R(E)^{W_{E}^{e}} \rightarrow R(E)$.

We define

$$
\Psi=\Psi^{W_{\bullet}^{e}}: R[W] \text {-modules } \longrightarrow R_{\mathrm{inv}} \text {-modules }
$$

by

$$
(\Psi M)(E):=M(E)^{W_{E}^{e}} .
$$

We note that $M(E)$ is an $R(E)$-module, and that passage to fixed points is lax symmetric monoidal, so that taking fixed points of the structure maps shows that $M(E)^{W_{E}^{e}}$ is an $R(E)^{W_{E}^{e}}$-module. Furthermore, if $E \supset F$ then the structure map $M(F) \rightarrow M(E)$ induces

$$
(\Psi M)(F)=M(F)^{W_{F}^{e}} \longrightarrow M(E)^{W_{E}^{e}}=(\Psi M)(E)
$$

since $W_{\bullet}^{e}$ is decreasing.

In the other direction, we may define

$$
\theta_{*}: R_{\mathrm{inv}} \text {-modules } \longrightarrow R \text {-modules }
$$

by termwise extension of scalars:

$$
\left(\theta_{*} N\right)(E)=R(E) \otimes_{R_{\text {inv }}(E)} N(E) .
$$

If $E \supset F$ we may define

$$
\begin{aligned}
\left(\theta_{*} N\right)(F)=R(F) \otimes_{R_{\text {inv }}(F)} N(F) & \longrightarrow R(E) \otimes_{R_{\text {inv }}(F)} N(E) \\
& \longrightarrow R(E) \otimes_{R_{\text {inv }}(E)} N(E)=\left(\theta_{*} N\right)(E) .
\end{aligned}
$$

Remark 5.8 The functor $\theta_{*}$ is a version of extension of scalars for diagrams. On the other hand coextension of scalars does not give a functor of diagrams in general.

The key result is as follows: 
Proposition 5.9 If $W_{\bullet}^{e}$ is a decreasing normal component structure, and $R$ is a $W-$ equivariant $\Sigma$-diagram of rings, taking fixed points under a normal component structure $W_{\bullet}^{e}$ gives a functor

$$
\Psi^{W_{\bullet}^{e}}: W \text {-equivariant- } R \text {-modules } \longrightarrow R_{\text {inv }} \text {-modules. }
$$

This has left adjoint $\theta_{*}$ given by termwise extension of scalars.

Provided $|W|$ is invertible in $R$, the unit map

$$
N(E) \stackrel{\cong}{\rightrightarrows}\left(R(E) \otimes_{R_{\text {inv }}(E)} N(E)\right)^{W_{E}^{e}}=\left(\Psi \theta_{*} N\right)(E)
$$

of the $\theta_{*} \vdash \Psi$ adjunction is an isomorphism.

Proof For the $\theta_{*} \vdash \Psi$ adjunction, note that objectwise we have

$$
\begin{aligned}
\operatorname{Hom}_{R(E)^{W_{E}^{e}}}\left(N(E), M(E)^{W_{E}^{e}}\right) & =\operatorname{Hom}_{R(E)^{W_{E}^{e}}}(N(E), M(E))^{W_{E}^{e}} \\
& =\operatorname{Hom}_{R(E)}\left(R(E) \otimes_{R(E)^{W_{E}^{e}}} N(E), M(E)\right)^{W_{E}^{e}}
\end{aligned}
$$

so that

$$
\operatorname{Hom}_{R_{\text {inv }}}(N, \Psi M)=\operatorname{Hom}_{R}(N, M)^{W_{\bullet}, e}
$$

as required.

The unit is an isomorphism since $N(E)$ and $R_{\text {inv }}(E)$ both have trivial $W_{E}^{e}$-action: one may take fixed points of the defining coequalizer, and use the fact that this is exact since $|W|$ is invertible.

\section{The algebraic model of toral $G$-spectra}

We are now ready to specialize the general discussion to the example arising from compact Lie groups. We will describe $\mathcal{A}(\mathbb{N}$, toral $)$ and $\mathcal{A}(G$, toral $)$ using enrichments of $\mathcal{A}(\mathbb{T})$. The starting point is $\Sigma_{a}(\mathbb{T})$ together with its action of $\mathbb{W} G$. We will add a little decoration to indicate which part of the isotropy group of $K$ is internal and which is external.

\section{A Decorating the poset}

We summarize the information we need about a subgroup $K$. These were discussed in detail in Sections 2 and 3:

- $H^{*}\left(B W_{G}^{e} K\right)$,

- $W_{G}^{d} K$,

- the action of $W_{G}^{d} K$ on $H^{*}\left(B W_{G}^{e} K\right)$. 
Equivalently we need:

- $(\mathbb{W} G)_{K}=(\mathbb{N} \cap N K) / \mathbb{T}=\mathbb{W} W K$,

- the action of $(\mathbb{W} G)_{K}$ on $\mathbb{T} / K$,

- the subgroup $(\mathbb{W} G)_{K}^{e}$ represented by elements of $\left(N_{G}(K)\right)_{e}$.

Recall from Lemma 2.2 that $(\mathbb{W} G)_{K}^{e}=\mathbb{W}\left(W_{G}^{e} K\right)$, and recall from Lemma 3.3 that $(\mathbb{W} G)_{K}^{d}=W_{G}^{d} K$ so that the second and third pieces of information give

$$
H^{*}\left(B W_{G}^{e} K\right)=H^{*}(B \mathbb{T} / K)^{\mathbb{W}\left(W_{G}^{e} K\right)} .
$$

The quotient group $W_{G}^{d} K=W K / W_{G}^{e} K=(\mathbb{W} W K) /\left(\mathbb{W}\left(W_{G}^{e} K\right)\right)$ then acts on the ring of invariants to give the twisted group ring.

Remark 6.1 We will also need this data with $K$ replaced by a flag $E=\left(K_{0} \supset \cdots \supset K_{S}\right)$. This is closely analogous, once we define

$$
N_{G}(E)=N_{G}\left(K_{0}\right) \cap \cdots \cap N_{G}\left(K_{s}\right) .
$$

Thus $W_{G}(E)=N_{G}(E) / K_{s}$,

$$
(\mathbb{W} G)_{E}=\left(\mathbb{N} \cap N_{G}(E)\right) / \mathbb{T}=(\mathbb{W} G)_{K_{0}} \cap \cdots \cap(\mathbb{W} G)_{K_{s}}
$$

and

$$
(\mathbb{W} G)_{E}^{e}=\mathbb{W} W_{G}^{e}(E) .
$$

It is clear that this again gives a normal component structure and

$$
(\mathbb{W} G)_{E}^{d}=W_{G}^{d}(E) \text {. }
$$

\section{B Structures from Lie groups}

The basis of the model is the diagram $\mathbb{R}_{a}$ of commutative rings defined on subgroups $K \subseteq \mathbb{T}$ by $\mathbb{R}_{a}(K)=H^{*}(B \mathbb{T} / K)$. For modules $M$ over $\mathbb{R}_{a}$ there are numerous structures: $\mathbb{W} G$ acts on rings, on Euler classes and on modules. Here we lay out how the structures interact with the group action with a view to showing it gives examples of $\mathbb{W} G$-equivariant diagrams in the sense of Section 4 above. At various times we will consider the poset $\Sigma$ to be either the poset $\Sigma_{a}(\mathbb{T})$ of closed subgroups of $\mathbb{T}$ and cotoral inclusions or the poset flag $\left(\Sigma_{a}(\mathbb{T})\right)$ of flags in $\Sigma_{a}(\mathbb{T})$ under inclusion.

The action gives the following structure:

- Conjugation by an element $w \in \mathbb{W} G$ gives a group homomorphism

$$
r_{w^{-1}}: K^{w}=w^{-1} K w \longrightarrow K
$$

and a map

$$
\bar{r}_{w^{-1}}: \mathbb{T} / K^{w} \longrightarrow \mathbb{T} / K
$$


- The conjugation in the previous bullet point gives a ring homomorphism $w_{m}=\left(\bar{r}_{w^{-1}}\right)^{*}: \mathbb{R}_{a}(K)=H^{*}(B \mathbb{T} / K) \longrightarrow H^{*}\left(B \mathbb{T} / K^{w}\right)=\mathbb{R}_{a}\left(K^{w}\right)$, with $(v w)_{m}=v_{m} w_{m}, e_{m}=1$.

If we define $w_{*} \mathbb{R}_{a}$ by $\left(w_{*} \mathbb{R}_{a}\right)(K)=\mathbb{R}_{a}\left(K^{w}\right)$, then we have a homomorphism of rings $w_{m}: \mathbb{R}_{a} \rightarrow w_{*} \mathbb{R}_{a}$. This composes by the rule $(v w)_{m}=v_{m} w_{m}, e_{m}=1$, so that we have an equivariant $\Sigma_{a}$-diagram of rings in the sense of Sections 4 and 5 .

- Pullback again gives a map $w_{m}: \operatorname{Rep}(G / K) \rightarrow \operatorname{Rep}\left(G / K^{w}\right)$ on representations. If $U^{H}=0$ then $\left(w_{*} U\right)^{H^{w}}=0$.

- By the previous bullet point, given $K \supseteq L$, pullback along the element $w$ maps Euler classes $\mathcal{E}_{H / K}$ to Euler classes $\mathcal{E}_{H^{w} / K^{w}}$.

- We may therefore define a new diagram $w_{*} \mathbb{R}_{a}$ of rings on the poset of flags by

$$
\left(w_{*} \mathbb{R}_{a}\right)\left(K_{0} \supset \cdots \supset K_{s}\right)=\mathbb{R}_{a}\left(K_{0}^{w} \supset \cdots \supset K_{s}^{w}\right)
$$

and we have ring maps $w_{m}: \mathbb{R}_{a} \rightarrow w_{*} \mathbb{R}_{a}$ satisfying $(v w)_{m}=v_{m} w_{m}, e_{m}=1$. We thus have a flag $\left(\Sigma_{a}\right)$-diagram of rings in the sense of Sections 4 and 5.

- Given a module $M$ over $\mathbb{R}_{a}$, we may define a module $w_{*} M$ over $w_{*} \mathbb{R}_{a}$ by

$$
\left(w_{*} M\right)\left(K_{0} \supset \cdots \supset K_{s}\right)=M\left(K_{0}^{w} \supset \cdots \supset K_{s}^{w}\right) .
$$

An equivariant module is given by module maps $w_{m}: M \rightarrow w_{*} M$ over the ring map $w_{m}: \mathbb{R}_{a} \rightarrow w_{*} \mathbb{R}_{a}$ with $(v w)_{m}=v_{m} w_{m}, e_{m}=1$.

\section{C Equivariant diagrams of rings}

The previous section shows that $\mathbb{R}_{a}$ is a $\mathbb{W} G$-equivariant flag $\left(\Sigma_{a}(\mathbb{T})\right)$-diagram of rings so we may apply the apparatus of Sections 4 and 5. This means that (WW $G)_{K}$ acts on $\mathbb{R}_{a}(K)$ by ring homomorphisms, and we may form the twisted group ring $\mathbb{R}_{a}(K)\left[(\mathbb{W} G)_{K}\right]$, or we may take invariants under $(\mathbb{W} G)_{K}^{e}=\mathbb{W} W_{G}^{e} K$ and then let $(\mathbb{W} G)_{K} /(\mathbb{W} G)_{K}^{e}=W_{G}^{d} K$ act by ring homomorphisms and form

$$
\mathbb{R}_{\mathrm{tw}}(K)=\mathbb{R}_{a}(K)^{\mathbb{W} W_{G}^{e} K}\left[W_{G}^{d} K\right]=H^{*}\left(B W_{G}^{e} K\right)\left[W_{G}^{d} K\right] .
$$

We observe that $\mathbb{R}_{a}$ extends to a flag $\left(\Sigma_{a}(\mathbb{T})\right)$-diagram of rings via

$$
\mathbb{R}_{a}\left(K_{0} \supset \cdots \supset K_{s}\right)=\mathcal{E}_{K_{0} / K_{s}}^{-1} H^{*}\left(B \mathbb{T} / K_{s}\right) .
$$

We have commented that the Lie component structure on $\operatorname{flag}\left(\Sigma_{a}\right)$ is normal. This allows us to extend $\mathbb{R}_{\text {inv }}$ to a flag $\left(\Sigma_{a}(\mathbb{T})\right)$-diagram with

$$
\mathbb{R}_{\mathrm{tW}}(F)=\mathbb{R}_{a}(F)^{\mathbb{W} W_{G}^{e} F}\left[W_{G}^{d} F\right] .
$$


It is worth making the values explicit.

Lemma 6.2 For any flag $F=\left(K_{0} \supset \cdots \supset K_{S}\right)$ we have

where

$$
\mathbb{R}_{\mathrm{tw}}(F)=\mathcal{E}_{K_{0} / K_{S}}^{-1} H^{*}\left(B W_{G}^{e} F\right)\left[W_{G}^{d} F\right]
$$

$$
\mathcal{E}_{K_{0} / K_{s}}=\left\{e(V) \mid V \in \operatorname{Rep}\left(W_{G}^{e} F\right), V^{K_{0}}=0\right\} .
$$

Proof Suppose $W$ is a finite group acting on a ring $R$ and $S$ is a multiplicatively closed set closed under the action of $W$. First, we note that inverting $S$ has the same effect as inverting the elements $N s=\prod_{w \in W} w s$, so that $S^{-1} M=(N S)^{-1} M$. Now observe

$$
\left(S^{-1} M\right)^{W}=\left((N S)^{-1} M\right)^{W}=(N S)^{-1}\left(M^{W}\right),
$$

where the second equality uses the fact we are in characteristic zero so that we may decompose $M$ into isotypical pieces and these will not interact.

Taking $W=\mathbb{W} W_{G}^{e} F, R=H^{*}\left(B \mathbb{T} / K_{S}\right)$ and $S=\mathcal{E}_{K_{0} / K_{s}, \mathbb{T}}$ this shows

$$
\mathbb{R}_{\text {inv }}(F)=\left(N \mathcal{E}_{K_{0} / K_{s}}\right)^{-1} H^{*}\left(B W_{G}^{e} F\right) .
$$

Finally, we consider the effects of inverting Euler classes. The weights of a chosen representation $V$ of $W_{G}^{e}(F)$ fall into $W$-orbits. If the decomposition into weights is $\left.V\right|_{\mathbb{T}}=\bigoplus_{i} \alpha_{i}$ we have

$$
e(V)=e\left(\bigoplus_{i} \alpha_{i}\right)=\prod_{i} e\left(\alpha_{i}\right) .
$$

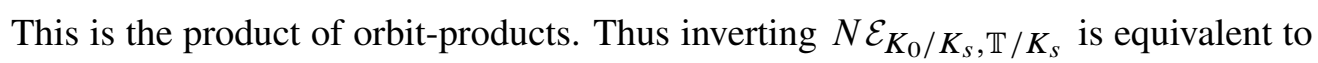
inverting $\mathcal{E}_{K_{0} / K_{s}, W_{G}^{e} F}$.

Warning 6.3 In the case of the torus, the ring $\mathbb{R}_{a}(K \supseteq L)$ is obtained from $\mathbb{R}_{a}(L)$ by localization (ie by inverting $\mathcal{E}_{K / L}$ ). This is not the case for $\mathbb{R}_{\mathrm{inv}}$. This is apparent even in the simple example of Section 6D.

\section{D The rotation group}

It is instructive to consider the example $G=\mathrm{SO}(3)$, with $\mathbb{N}=O(2), \mathbb{T}=\mathrm{SO}(2)$. We will display various data associated to a length-1 flag in rows. The first row is a module $N$ over $\mathbb{R}_{a}$, the next pair of rows gives $\mathbb{R}_{a}$, followed by the component structure $W_{\bullet}^{e}$, a pair of rows for $\mathbb{R}_{\mathrm{inv}}$ and finally an $\mathbb{R}_{\mathrm{inv}}-$ module $M$. 
We illustrate the structure for the particular flag $F=(\mathbb{T} \supset 1)$ and its two length- 0 subflags in the following diagram:

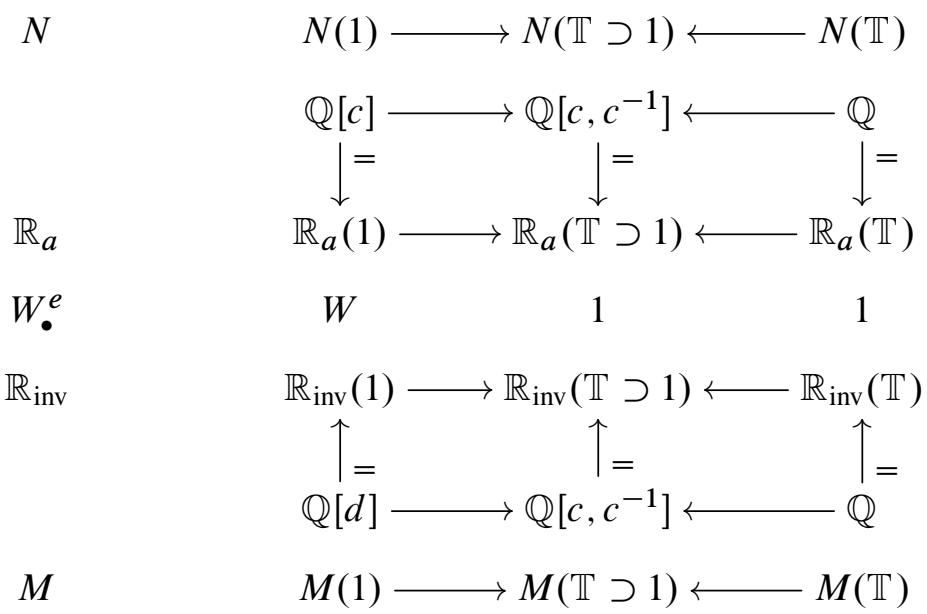

Take particular note of the fourth row, where we record the Weyl groups of $W_{G}^{e} K$ and $W_{G}^{e}(\mathbb{T} \subseteq K$ ), using the abbreviation $W=\mathbb{W} G$ (a reflection group of order 2). We will use this example in describing the functors relating $\mathcal{A}(G$, toral) and $\mathcal{A}(\mathbb{N}$, toral $)$, so that $N$ is an equivariant $\mathbb{R}_{a}$-module, potentially in $\mathcal{A}\left(\mathbb{N}\right.$, toral) and $M$ is an $\mathbb{R}_{\text {inv }}$-module, potentially in $\mathcal{A}(G$, toral $)$. As elsewhere $H^{*}(B \mathrm{SO}(2))=\mathbb{Q}[c]$ for an element $c$ of codegree 2 and $H^{*}(B \mathrm{SO}(3))=\mathbb{Q}[d]$ for an element $d=c^{2}$ of codegree 4. Note in particular that

$$
\mathbb{R}_{\text {inv }}(\mathbb{T} \supset 1)=\mathbb{Q}\left[c, c^{-1}\right] \neq \mathbb{Q}\left[d, d^{-1}\right]=\mathcal{E}_{\mathbb{T}}^{-1} \mathbb{R}_{\text {inv }}(1) .
$$

We should also consider the flag $\mathbb{T} \subset C_{r}$ for $r \geq 2$ so as to note the differences entailed by the fact that $W\left(C_{r}\right)=\mathbb{W} G$ is discrete and hence has trivial identity component:

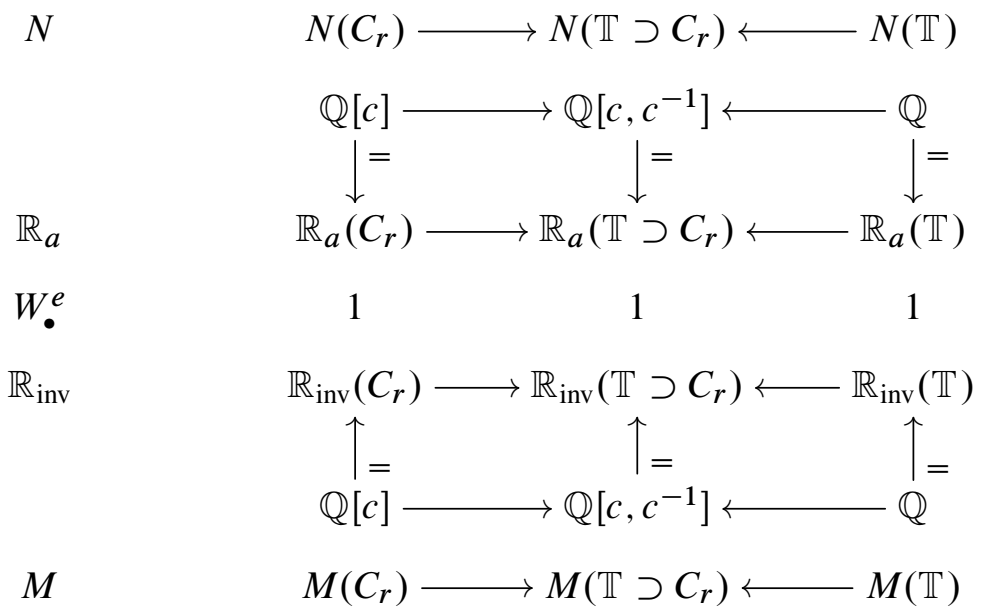




\section{E Subcategory conditions for $G$}

The algebraic model $\mathcal{A}(\mathbb{T})$ is the category of modules over the diagram $\mathbb{R}_{a}$ of rings which are subject to the following three conditions: (i) quasicoherence, (ii) extendedness and (iii) $\mathcal{F}$-continuity.

In view of Warning 6.3, we must be explicit in formulating the quasicoherence and extendedness conditions for equivariant $\mathbb{R}_{\mathrm{inv}}$-modules on the poset $\operatorname{flag}\left(\Sigma_{a}\right)$. We also observe that the conditions are compatible with the $\mathbb{W} G$-action.

Suppose then that we have flags

$$
E=\left(K_{0} \supset K_{1} \supset \cdots \supset K_{S}\right) \text { and } F=\left(L_{0} \supset L_{1} \supset \cdots \supset L_{t}\right)
$$

with $E \supset F$. This gives a ring map

$$
\mathbb{R}_{\text {inv }}(F) \longrightarrow \mathbb{R}_{\text {inv }}(E),
$$

and for any $\mathbb{R}_{\text {inv }}$-module $M$ we have a structure map $M(F) \rightarrow M(E)$.

In order to discuss quasicoherence and extendedness, we introduce further terminology. This will be shown to be redundant, and not be used after this subsection.

Definition 6.4 (i) An $\mathbb{R}_{\mathrm{inv}}$-module $M$ follows the coefficients if for any pair of flags $E \supset F$ the structure map induces an isomorphism

$$
\mathbb{R}_{\text {inv }}(E) \otimes_{\mathbb{R}_{\text {inv }}(F)} M(F) \cong M(E) .
$$

(ii) An $\mathbb{R}_{\mathrm{inv}}-$ module $M$ is quasicoherent if it follows the coefficients whenever $F=\left(K_{S}\right)$ is the singleton flag of the smallest term in $E$ :

$$
\mathbb{R}_{\text {inv }}(E) \otimes_{\mathbb{R}_{\text {inv }}\left(K_{S}\right)} M\left(K_{S}\right) \cong M(E) .
$$

(iii) An $\mathbb{R}_{\text {inv }}$-module $M$ is extended if it follows the coefficients whenever $F=\left(K_{0}\right)$ is the singleton flag of the largest term in $E$ :

$$
\mathbb{R}_{\mathrm{inv}}(E) \otimes_{\mathbb{R}_{\text {inv }}\left(K_{0}\right)} M\left(K_{0}\right) \cong M(E) .
$$

Remark 6.5 (i) If $M$ is qce then it follows the coefficients for any inclusion $E \supseteq F$ of flags.

(ii) If $M$ follows the coefficients for the addition of any single term to a flag then it is qce and follows the coefficients in general.

(iii) However if $M$ is qce for pairs this is not sufficient on its own. For example we may consider the inclusion of a length-1 flag in a length-2 flag: $(H \supset L) \rightarrow(H \supset K \supset L)$. 
In this case, $\mathbb{W}_{(H \supset K \supset L)}$ is typically a proper subgroup of $\mathbb{W}_{(H \supset L)}$ and so in general we have a proper containment

$$
\begin{aligned}
\mathbb{R}_{a}(H \supset K \supset L)^{\mathbb{W}_{(H \supset L)}} & =\mathcal{E}_{H / L}^{-1} \mathbb{R}_{a}(L)^{\mathbb{W}_{(H \supset L)}} \\
& \subseteq \mathcal{E}_{H / L}^{-1} \mathbb{R}_{a}(L)^{\mathbb{W}_{(H \supset K \supset L)}}=\mathbb{R}_{a}(H \supset K \supset L)^{\mathbb{W}_{(H \supset K \supset L)}} .
\end{aligned}
$$

The condition

$$
M(H \supset K \supset L)=\mathbb{R}_{a}(H \supset K \supset L)^{\mathbb{W}_{(H \supset K \supset L)}} \otimes_{\mathbb{R}_{a}(H \supset K \supset L)^{\mathbb{W}_{(H \supset L)}}} M(H \supset L)
$$

is a new condition, one not seen in the inclusion of a length- 0 flag in a length- 1 flag.

The idea of $\mathcal{F}$-continuity is that it provides a uniform bound on denominators. In the original setting, the definition is that $\mathcal{F}$-continuity requires a specified factorization for each subgroup $K$

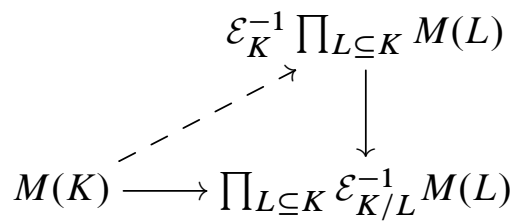

and these should be compatible with composition. We note that the collection of subgroups involved in this condition depends $\mathbb{W} G$-equivariantly on $K$, and if the condition holds for $K$ it holds for any subgroup in the $\mathbb{W} G$-orbit of $K$.

We may now formulate the condition for $\mathbb{R}_{\text {inv }}$-modules. The equivariance will ensure that maps have images in modules of invariants, so we avoid the use of invariants in the statement.

Definition 6.6 An $\mathbb{R}_{\text {inv }}$-module $M$ is $\mathcal{F}$-continuous if there is a specified factorization for each subgroup $K$

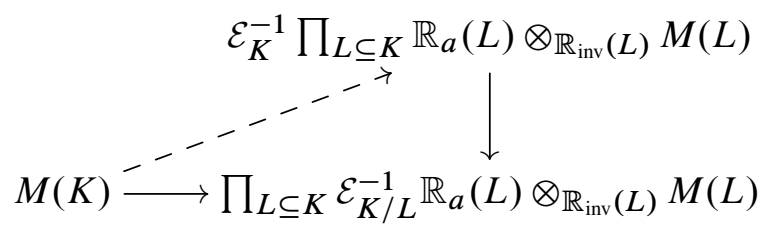

and these should be compatible with composition.

\section{F The model}

We are now ready to define the algebraic model $\mathcal{A}(G$, toral $)$. Throughout this subsection we use the diagram $\Sigma=\operatorname{flag}\left(\Sigma_{a}(\mathbb{T})\right)$ and $\mathbb{R}_{a}$ is viewed as a $\Sigma$-diagram of rings. 
Definition 6.7 (i) The category of $\mathbb{R}_{a}[\mathbb{W} G]$-modules is the category of $\mathbb{W} G-$ equivariant $\mathbb{R}_{a}$-modules. In view of Lemma 5.6 we will not distinguish between the model in which these are $\Sigma$-diagrams with the additional structure of a $\mathbb{W} G$-action and the model in which they are $\Sigma \rtimes \mathbb{W} G$-diagrams.

(ii) The category $\mathcal{A}(\mathbb{T})[\mathbb{W} G]$ is the category of qce, $\mathcal{F}$-continuous $\mathbb{W} G$-equivariant $\mathbb{R}_{a}$-modules.

Now consider the Lie group component structure ( $\mathbb{W} G)_{\bullet}^{e}$ on $\mathbb{W} G \rtimes \Sigma$ and the quotient $(\mathbb{W} G)_{\text {. }}^{d}$ (see Section 4E). This gives two diagrams of rings. Firstly, we have the

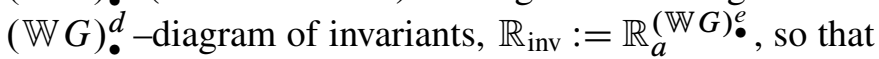

$$
\mathbb{R}_{\text {inv }}(K)=H^{*}\left(B W_{G}^{e} K\right) .
$$

Secondly, we have the $\Sigma$-diagram $\mathbb{R}_{\text {tw }}$ of twisted group rings, whose value at a subgroup $K$ is

$$
\mathbb{R}_{\mathrm{tw}}(K)=H^{*}\left(B W_{G}^{e} K\right)\left[W_{G}^{d} K\right]
$$

Definition 6.8 (i) The category $\mathcal{A}_{\text {inv }}(G$, toral) is the category of qce, $\mathcal{F}$-continuous $\mathbb{R}_{\text {inv }}$-modules.

(ii) The category $\mathcal{A}_{\mathrm{tw}}(G$, toral) is the category of qce, $\mathcal{F}$-continuous modules over the diagram $\mathbb{R}_{\mathrm{tw}}$ of rings.

Remark 6.9 By Lemma 5.7, $\mathcal{A}_{\text {inv }}(G$, toral $) \simeq \mathcal{A}_{\mathrm{tw}}(G$, toral $)$, and as a matter of style we view $\mathcal{A}_{\text {inv }}(G$, toral $)$ as the primary one, abbreviating it to $\mathcal{A}(G$, toral $)$.

There is one special case where it is easy to describe the model of toral spectra.

Lemma 6.10 The model for toral spectra simplifies when the identity component is a torus to give

$$
\mathcal{A}(\mathbb{N}, \text { toral })=\mathcal{A}(\mathbb{T})[\mathbb{W} G]
$$

Proof We need only observe that if $K \subseteq \mathbb{T}$ then the identity component of $W_{\mathbb{N}} K$ is a torus, and so it has trivial Weyl group. The component structure is therefore the trivial one, and $\mathbb{R}_{\text {inv }}=\mathbb{R}_{a}$.

\section{Toral $G$-spectra and toral $\mathbb{N}$-spectra}

We consider the algebraic counterpart of restriction from $G$-spectra to $\mathbb{N}$-spectra, and its right adjoint. We know from [15] that the category of $\mathbb{T}$-spectra is modelled by $\mathcal{A}(\mathbb{T})$. It is rather clear (and made explicit in Lemma 11.1) that the module $M=\pi_{*}^{\mathcal{A}}(X)$ in $\mathcal{A}(\mathbb{T})$ arising from a $G$-spectrum $X$ is a $\mathbb{W} G$-equivariant module. 
Our model $\mathcal{A}(G$, toral $)$ has the property that restriction from $G$-spectra to $\mathbb{N}$-spectra is modelled by the functor $\theta_{*}$ defined in Section $5 \mathrm{C}$. The purpose of this section is to establish that the descent adjunction (Proposition 5.9) relating $\mathbb{R}_{\mathrm{inv}}$-modules and equivariant $\mathbb{R}_{a}$-modules continues to hold for the subcategories of qce, $\mathcal{F}$-continuous modules.

\section{A From $\mathcal{A}(G$, toral $)$ to $\mathcal{A}(\mathbb{T})[\mathbb{W} G]$}

First we consider the algebraic counterpart of restriction.

\section{Proposition 7.1 The functor}

$$
\theta_{*}: \mathbb{R}_{\mathrm{inv}} \text {-modules } \longrightarrow \mathbb{W} G \text {-equivariant- } \mathbb{R}_{a} \text {-modules }
$$

preserves quasicoherence, extendedness and $\mathcal{F}$-continuity and hence induces a functor

$$
\theta_{*}: \mathcal{A}(G, \text { toral }) \longrightarrow \mathcal{A}(\mathbb{T})[\mathbb{W} G]
$$

Proof Suppose $M$ is an $\mathbb{R}_{\text {inv }}$-module with image $\theta_{*} M$ defined on a flag $F$ by

$$
\left(\theta_{*} M\right)(F)=\mathbb{R}_{a}(F) \otimes_{\mathbb{R}_{\text {inv }}(F)} M(F) .
$$

We note that $\mathbb{R}_{\text {inv }}(F)=\mathbb{R}_{a}(F)^{(\mathbb{W} G)_{F}^{e}}$, and $\mathbb{R}_{a}(F)$ is free over $\mathbb{R}_{\text {inv }}(F)$. As in Lemma 6.2, we note that a multiplicatively closed set $S$ preserved by the action of a finite group has a cofinal multiplicatively closed subset $N S$ whose elements are the products $N s$ over orbits. Thus we will assume that the multiplicatively closed subsets are invariant. Since $\theta_{*} M$ lies over $\mathbb{N}$ the component structure is trivial so the $\mathbb{W} G$-action is entirely through equivariance (no invariants are involved). Accordingly, it suffices to verify quasicoherence and extendedness for pairs rather than more general flags. We will write the proof in those terms since the subgroups concerned appear more directly.

If $M$ is quasicoherent then the condition on cotoral pairs is that the natural map induces an isomorphism $\mathbb{R}_{\text {inv }}(K \supseteq L) \otimes_{\mathbb{R}_{\text {inv }}(L)} M(L)=M(K \supseteq L)$. It follows that

$$
\begin{aligned}
\left(\theta_{*} M\right)(K \supseteq L) & =\mathbb{R}_{a}(K \supseteq L) \otimes_{\mathbb{R}_{\text {inv }}(K \supseteq L)} M(K \supseteq L) \\
& =\mathbb{R}_{a}(K \supseteq L) \otimes_{\mathbb{R}_{\text {inv }}(K \supseteq L)} \mathbb{R}_{\text {inv }}(K \supseteq L) \otimes_{\mathbb{R}_{\text {inv }}(L)} M(L) \\
& =\mathbb{R}_{a}(K \supseteq L) \otimes_{\mathbb{R}_{\text {inv }}(L)} M(L) \\
& =\mathbb{R}_{a}(K \supseteq L) \otimes_{\mathbb{R}_{a}(L)} \mathbb{R}_{a}(L) \otimes_{\mathbb{R}_{\text {inv }}(L)} M(L) \\
& =\mathbb{R}_{a}(K \supseteq L) \otimes_{\mathbb{R}_{a}(L)}\left(\theta_{*} M\right)(L)
\end{aligned}
$$

and $\theta_{*} M$ is also quasicoherent. 
If $M$ is extended then

$$
\mathbb{R}_{\text {inv }}(K \supseteq L) \otimes_{\mathbb{R}_{\text {inv }}(K)} M(K)=M(K \supseteq L) .
$$

For $\theta_{*} M$ we may then calculate

$$
\begin{aligned}
\mathbb{R}_{a}(K \supseteq L) \otimes_{\mathbb{R}_{a}(K)}\left(\theta_{*} M\right. & (K) \\
& =\mathbb{R}_{a}(K \supseteq L) \otimes_{\mathbb{R}_{a}(K)} \mathbb{R}_{a}(K) \otimes_{\mathbb{R}_{\text {inv }}(K)} M(K) \\
& =\mathbb{R}_{a}(K \supseteq L) \otimes_{\mathbb{R}_{\text {inv }}(K)} M(K) \\
& =\mathbb{R}_{a}(K \supseteq L) \otimes_{\mathbb{R}_{\text {inv }}(K \supseteq L)}\left(\mathbb{R}_{\text {inv }}(K \supseteq L) \otimes_{\mathbb{R}_{\text {inv }}(K)} M(K)\right) \\
& =\mathbb{R}_{a}(K \supseteq L) \otimes_{\mathbb{R}_{\text {inv }}(K \supseteq L)} M(K \supseteq L) \\
& =\left(\theta_{*} M\right)(K \supseteq L) .
\end{aligned}
$$

Thus $\theta_{*} M$ is also extended.

Suppose that $M$ is $\mathcal{F}$-continuous. Since $\mathbb{R}_{a}(F)$ is free of finite rank over $\mathbb{R}_{\text {inv }}(F)$, we may form the diagram:

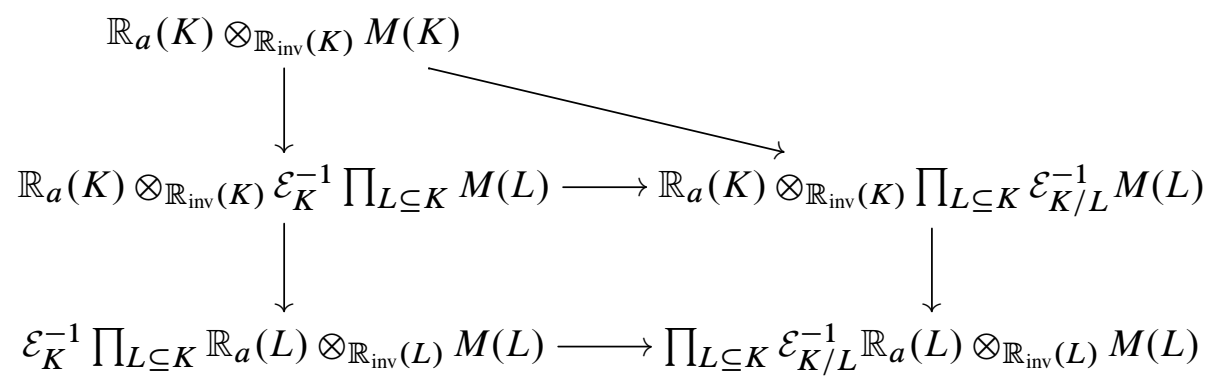

The two bottom verticals are induced by the $\mathbb{R}_{\text {inv }}(L)$-maps

$$
M(L) \longrightarrow \mathbb{R}_{a}(L) \otimes_{\mathbb{R}_{\text {inv }}(L)} M(L)
$$

using the universal property of products. The diagram shows that $\theta_{*} M$ is also $\mathcal{F}$-continuous.

We will show in Proposition 11.8 that $\theta_{*}$ fits into a diagram:

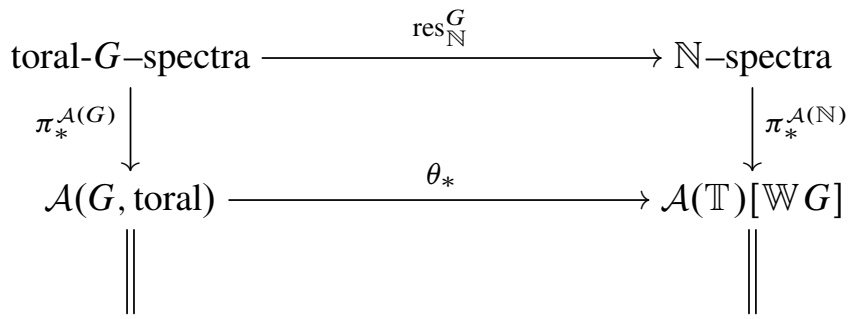

$\mathcal{F}$-cts-qce- $\mathbb{R}_{\text {inv }}-$ modules $\longrightarrow \mathbb{W} G$-equivariant- $\mathcal{F}$-cts-qce- $\mathbb{R}_{a}-$ modules. 
We conjecture that these maps to abelian categories can be upgraded to Quillen equivalences with the associated differential graded objects.

\section{B Normal modules}

By contrast with $\theta_{*}$, the fact that the functor $\Psi$ takes qce modules to qce modules is rather subtle. Consider for instance the quasicoherence associated to a cotoral inclusion $K \supseteq L$. If the $\mathbb{W} G$-equivariant $\mathbb{R}_{a}$-module $N$ is quasicoherent, then $N(K \supseteq L)=\mathcal{E}_{K / L}^{-1} N(L)$. We may take $(\mathbb{W} G)_{K \supseteq L}$-invariants of both sides, but since $(\mathbb{W} G)_{K \supseteq L}$ may be a proper subgroup of $(\mathbb{W} G)_{L}$ this is not the quasicoherence condition for $\Psi N$, which states instead that

$$
N(K \supseteq L)^{(\mathbb{W} G)_{K \supseteq L}}=\mathcal{E}_{K / L}^{-1} \mathbb{R}_{a}(L)^{(\mathbb{W} G)_{K \supseteq L}} \otimes_{\mathbb{R}_{a}(L)^{(\mathbb{W} G)_{L}}} N(L)^{(\mathbb{W} G)_{L}} .
$$

In effect we need to be able to reconstruct modules from their invariants using the ring $\mathbb{R}_{a}$. This is a special property not enjoyed by all modules.

We suppose then that $W$ is a finite group acting on a $\mathbb{Q}$-algebra $R$.

Definition 7.2 We say that a $W$-equivariant $R$-module $M$ is normal if the natural map

$$
\nu: R \otimes_{R^{W}} M^{W} \longrightarrow M
$$

is an isomorphism.

It is worth noting that normality is a strong condition.

Example 7.3 (i) Clearly if $R=\mathbb{Q} G$ and $M$ is a nontrivial simple module then $M$ is not normal.

(ii) This also happens for modules that arise in our setting. For instance we may take $R=H^{*}(B \mathrm{SO}(2))=\mathbb{Q}[c]$ with $W$ of order 2 acting to negate $c$, so that $R^{W}=H^{*}(B \mathrm{SO}(3))=\mathbb{Q}[d]$ with $d=c^{2}$. However it is easy to see the ideal $M=(c)$ is not normal (for example because the inclusion $\left(c^{2}\right) \subseteq(c)$ is an isomorphism on $W$-fixed points and the free module $\left(c^{2}\right)$ is normal). The fact that will give the conclusion we need is that if $d$ is inverted everywhere (so $R=\mathbb{Q}\left[c, c^{-1}\right]=M$ ) then we do obtain a normal module.

There is an easy positive result.

Lemma 7.4 If $R$ is free over $R^{W}$ then the class of normal $R$-modules is closed under the following operations: 
- arbitrary sums,

- passage to kernels,

- passage to cokernels,

- passage to extensions.

Any extended module of the form $M=R \otimes_{S} M^{\prime}$ with $W$ acting trivially on $S$ and $M^{\prime}$ is normal.

Proof For example if $F_{0}, F_{1}$ are normal and $M$ is the cokernel of a map $F_{1} \rightarrow F_{0}$ we may form the diagram:

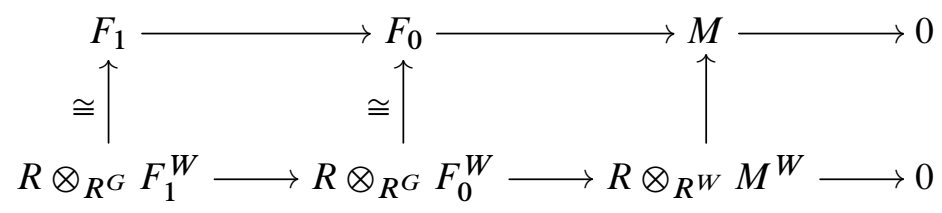

Because we are in characteristic 0 , passage to $W$-fixed points is exact, and by hypothesis $R$ is flat over $R^{W}$, so the isomorphism follows from the short 5-lemma.

The other cases are similar. For an extended module of the given form, we have $M^{W}=\left(R \otimes_{S} M^{\prime}\right)^{W}=R^{W} \otimes_{S} M^{\prime}$ and normality is clear.

We will show that the modules that occur in an object $N$ of $\mathcal{A}(\mathbb{N}$, toral) are close enough to being normal to ensure that $\Psi N$ is qce. The following examples show that this is somewhat less restrictive than might be expected.

Example 7.5 (i) We have seen that $H^{*}(B \mathrm{SO}(2))=\mathbb{Q}[c]$ is a free module over $H^{*}(B \mathrm{SO}(3))=\mathbb{Q}[d]$. More precisely

$$
\mathbb{Q}[c]=\mathbb{Q}[d] \otimes\left(\epsilon \oplus \Sigma^{2} \sigma\right)
$$

where $\epsilon$ is the trivial module and $\sigma$ is the sign module. If we ignore grading then $\mathbb{Q}[c]=\mathbb{Q}[d][W]$.

In any case it will follow by decomposing $V$ into $W$-isotypical pieces that any $\mathbb{Q}[c]$-module of the form $\mathbb{Q}\left[c, c^{-1}\right] \otimes V$ is normal.

The relevance of this is that it shows the model of $\{1, \mathbb{T}\}-S O(3)$-spectra (ie of spectra with geometric isotropy in $\{1, \mathbb{T}\})$ behaves well. Indeed, we may consider an object

$$
X=\left(N \stackrel{\beta}{\longrightarrow} \mathbb{Q}\left[c, c^{-1}\right] \otimes V\right)
$$


of the model of $\{1, \mathbb{T}\}-\mathbb{N}$-spectra; this means $N$ is a $\mathbb{Q}[c][W]$-module and $V$ is a $\mathbb{Q}[W]$-module with the map $\beta$ being inversion of $c$. By the above argument, $N$ is normal, and it follows that

$$
\Psi X=\left(N^{W} \longrightarrow \mathbb{Q}\left[c, c^{-1}\right] \otimes V\right)
$$

is qce.

(ii) Similarly for the rank-2 group SU(3) with maximal torus ST(3) and Weyl group $\mathbb{W} G=\Sigma_{3}$, where

$$
H^{*}(B \mathrm{ST}(3))=H^{*}(B \mathrm{SU}(3)) \otimes\left(\epsilon \oplus \Sigma^{2} \mu \oplus \Sigma^{4} \mu \oplus \Sigma^{6} \sigma\right),
$$

where $\sigma$ is the nontrivial simple representation of degree 1 and $\mu$ that of degree 2 . If we ignore the grading then

$$
H^{*}(B \mathrm{ST}(3))=H^{*}(B \mathrm{SU}(3))[\mathbb{W} G] .
$$

One may check that if we invert linear forms any module of the form $H^{*}(B \mathrm{ST}(3)) \otimes V$ is normal (the case $V=\mu$ is most interesting).

It seems natural to expect that with linear forms inverted, the module $H^{*}(B \mathbb{T}) \otimes V$ is normal for any compact Lie group $G$, and it may be that more general statements could be formulated giving the result that $\Psi N$ is qce directly as was done for $G=\mathrm{SO}$ (3) in the above example. However, we will instead use injective resolutions to reduce the verification to special cases.

\section{C From $\mathcal{A}(\mathbb{T})[\mathbb{W} G]$ to $\mathcal{A}(G$, toral $)$}

After our discussion of normal modules we are equipped to turn to the right adjoint $\Psi$.

Proposition 7.6 The functor

$$
\Psi: \mathbb{W} G \text {-equivariant- } \mathbb{R}_{a} \text {-modules } \longrightarrow \mathbb{R}_{\mathrm{inv}} \text {-modules }
$$

takes quasicoherent extended modules to quasicoherent extended modules and preserves $\mathcal{F}$-continuous modules and hence induces a functor

$$
\Psi: \mathcal{A}(\mathbb{T})[\mathbb{W} G] \longrightarrow \mathcal{A}(G, \text { toral }) .
$$

Remark 7.7 The functor $\Psi$ does not preserve quasicoherence or extendedness separately. 
Proof First, $\mathcal{F}$-continuity is straightforward, since $M(K)^{(\mathbb{W} G)_{K}}$ maps into the $(\mathbb{W} G)_{K}$-invariants inside the $(\mathbb{W} G)_{K \supseteq L}$-invariants, and we have already observed that the passage to invariants commutes with products and localizations. The main issue is the qce property, which is rather delicate.

Suppose $N$ is an $\mathbb{R}_{a}$-module with image $\Psi N$ defined by

$$
(\Psi N)(F)=N(F)^{(\mathbb{W} G)_{F}^{e} .}
$$

As in Lemma 6.2, we note that a multiplicatively closed set $S$ preserved by the action of a finite group has a cofinal multiplicatively closed subset $N S$ whose elements are the products $N s$ over orbits. Thus we will assume that the multiplicatively closed subsets are invariant.

Now suppose $E \supseteq F$. Since $N$ is qce we have

$$
N(E)=\mathbb{R}_{a}(E) \otimes_{\mathbb{R}_{a}(F)} N(F) .
$$

Taking fixed points under $(\mathbb{W} G)_{E}^{e}$ we have

$$
(\Psi N)(E)=N(E)^{(\mathbb{W} G)_{E}^{e}}=\left[\mathbb{R}_{a}(E) \otimes_{\mathbb{R}_{a}(F)} N(F)\right]^{(\mathbb{W} G)_{E}^{e}} .
$$

Since the connected structure is decreasing, $(\mathbb{W} G)_{E}^{e} \subseteq(\mathbb{W} G)_{F}^{e}$ and we need to show the natural map

$$
\begin{aligned}
v_{E \supset F}: \mathbb{R}_{\mathrm{inv}}(E) \otimes_{\mathbb{R}_{\mathrm{inv}}(F)}(\Psi N)(F)= & \mathbb{R}_{a}(E)^{(\mathbb{W} G)_{E}^{e}} \otimes_{\mathbb{R}_{a}(F)^{(\mathbb{W} G)_{F}}} N(F)^{(\mathbb{W} G)_{F}^{e}} \\
& \longrightarrow\left[\mathbb{R}_{a}(E) \otimes_{\mathbb{R}_{a}(F)} N(F)\right]^{(\mathbb{W} G)_{E}^{e}}=(\Psi N)(E)
\end{aligned}
$$

is an isomorphism.

The character of the problem is like that of normality, and we adopt a similar strategy. We first note that the question of whether $\nu_{E \supset F}$ is an isomorphism only depends on $N$ only through $N(F)$, which is a $(\mathbb{W} G)_{F}$-equivariant $\mathbb{R}_{a}(F)$-module.

Lemma 7.8 The class of modules $N(F)$ for which $v$ is an isomorphism is closed under the following operations:

- arbitrary sums,

- passage to kernels,

- passage to cokernels,

- passage to extensions.

It is an isomorphism for $N(F)=\mathbb{R}_{a}(F)$. 
Proof This is clear for sums, and it is clear for $N(F)=\mathbb{R}_{a}(F)$. We illustrate the other cases by the passage to kernels. Suppose then that $v$ is an isomorphism for $N(F)=B, C$ and that we have an exact sequence

$$
0 \longrightarrow A \longrightarrow B \longrightarrow C \text {. }
$$

Taking $\mathbb{W}_{F}=(\mathbb{W} G)_{F}$-invariants, and tensoring with $\mathbb{R}_{a}(E)^{\mathbb{W}_{E}}$ over $\mathbb{R}_{a}(F)^{\mathbb{W}_{F}}$ we obtain the first row in the following diagram, and similarly the second row is obtained by tensoring with $\mathbb{R}_{a}(E)$ and taking $\mathbb{W}_{E}$-invariants:

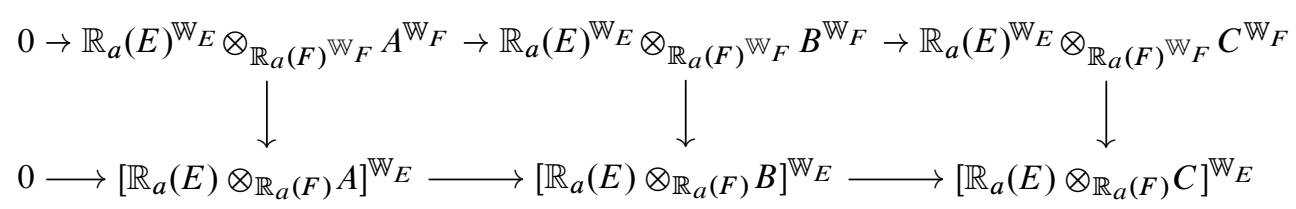

The result follows from the short 5-lemma.

In effect the lemma says the result is only obvious when $N(F)$ is a free $\mathbb{R}_{a}(F)$-module. The strategy of proof is to reduce to the case of certain standard injectives that we identify precisely. We note that these standard injectives come from the polynomial rings $H^{*}(B \mathbb{T} / K)$. Because the polynomial ring $H^{*}(B \mathbb{T} / K)$ is Gorenstein, the injective is also the local cohomology and we can deduce this case from that of the free module.

In more detail, we show in Section 8 that any module $N$ admits an injective presentation $0 \rightarrow N \rightarrow I_{0} \rightarrow I_{1}$ where $I_{0}$ and $I_{1}$ are sums of $\mathbb{W} G$-equivariant injectives of a particular form. It therefore suffices to prove the result for the special case of these basic injectives. These are discussed in detail in Section 8 , but we will summarize the properties we need here to avoid interrupting the thread of the argument.

Suppose then that $K \subseteq \mathbb{T}$ and consider a basic injective arising from $K$. This is obtained from an injective module $I$ over $H^{*}(B \mathbb{T} / K)$, namely

$$
I=H_{*}\left(B \mathbb{T} / K^{L \mathbb{T} / K}\right) .
$$

Indeed, the right adjoint $f_{K}^{\mathbb{T}}$ to evaluation at $K$ gives an injective $f_{K}^{\mathbb{T}}(I)$ in $\mathcal{A}(\mathbb{T})$ and then we may coinduce the module to $\mathbb{N}$, where it takes the form

$$
f_{(K)}^{\mathbb{N}}(\mathbb{Q}[W] \otimes I)=f_{(K)}^{\mathbb{N}}(I) \otimes \mathbb{Q}[W]
$$

in $\mathcal{A}(\mathbb{N}$, toral $)=\mathcal{A}(\mathbb{T})[\mathbb{W} G]$. Notice that the value of this injective at any flag is free over $\mathbb{Q}[W]$.

Of course $N(H)=0$ unless $H$ is subconjugate to $K$. From the qce condition it follows that the value $N(F)$ is zero unless $K \supseteq L_{0}$. We note that if $K \nsubseteq K_{0}$ then 
$I$ is $\mathcal{E}_{K_{0} / L_{0}}$ torsion; as observed elsewhere, we can always localize with respect to products over $W$-orbits. Thus the qce condition for $\Psi N$ holds for such flags. We may therefore suppose that $K \supseteq K_{0}$.

We may be explicit about the value of $N(F)$. Indeed, $K \supseteq L_{0} \supseteq \cdots \supseteq L_{t}$ and $\mathbb{T} / L_{t}=\mathbb{T} / K \times K / L_{t}$. Thus

$$
\begin{aligned}
N(F) & =\mathcal{E}_{L_{0} / L_{S}}^{-1} H^{*}\left(B \mathbb{T} / L_{S}\right) \otimes_{H^{*}(B \mathbb{T} / A)} H_{*}\left(B(\mathbb{T} / K)^{L(\mathbb{T} / K)}\right)[\mathbb{W} G] \\
& =\mathcal{E}_{L_{0} / L_{S}}^{-1} H^{*}\left(B K / L_{S}\right) \otimes_{\mathbb{Q}} H_{*}\left(B(\mathbb{T} / K)^{L(\mathbb{T} / K)}\right)[\mathbb{W} G] .
\end{aligned}
$$

It remains to observe that $v_{E \supset F}$ is an isomorphism for this $N(F)$. We will first verify the statement without $\mathbb{W} G$. For this we apply the following lemma to $T=\mathbb{T} / K$.

Lemma 7.9 Suppose $\mathfrak{W}$ is any finite subgroup of $\operatorname{Aut}(T)$, and consider the category of $\mathfrak{W}$-equivariant $H^{*}(B T)$-modules.

If $B T^{L T}$ is the Thom space of the tangent space $L T$ of $T$ at $e$, the module $H_{*}\left(B T^{L T}\right)$ is the cohomology of a finite complex of $H^{*}(B T)[\mathfrak{W}]$-modules each term of which is generated by $H^{*}(B T)$ using direct sums, cokernels and direct limits.

Remark 7.10 Note that the insertion of the adjoint representation $L T$ is necessary. For example if $T=\mathrm{SO}(2)$ is the circle and $W=\mathbb{W} \mathrm{SO}(3)$ is of order $2, H_{*}\left(B T^{L T}\right)$ is a suspension of the dual of $(c)$, and we have the exact sequence

$$
0 \longrightarrow \mathbb{Q}[c] \longrightarrow \mathbb{Q}\left[c, c^{-1}\right] \longrightarrow(c)^{\vee} \longrightarrow 0
$$

proving the lemma in this case. On the other hand the module $k[c]^{\vee}$ is not in this category since $\theta_{*} \Psi\left(k[c]^{\vee}\right) \not k[c]^{\vee}$.

Proof To start with, ignore the action of $\mathfrak{W}$. If we choose a finite set $\mathfrak{G}$ of generators of the ideal $\mathfrak{m}$ of elements of $R=H^{*}(B T)$ of positive codegree, we may form the stable Koszul complex $K_{\infty}^{\bullet}(\mathfrak{G})$, with

$$
K_{\infty}^{n}(\mathfrak{G})=\bigoplus_{\tau \subseteq \mathfrak{G},|\tau|=n} R\left[\frac{1}{\prod_{g \in \tau} g}\right] .
$$

The point of the stable Koszul complex is that it calculates local cohomology, so that if $T$ is of rank $s$, we have

$$
H^{*}\left(K_{\infty}^{\bullet}(\mathfrak{G})\right)=H_{\mathfrak{m}}^{*}(R)=H_{\mathfrak{m}}^{s}(R)=H_{*}\left(B T^{L T}\right) .
$$

Now choose $\mathfrak{G}$ so that the construction is $\mathfrak{W}$-equivariant. Indeed, adding translates as necessary, we choose $\mathfrak{G}$ to be a union of $\mathfrak{W}$-orbits, and group the terms in $K_{\infty}^{n}(\mathfrak{G})$ 
into $\mathfrak{W}$-orbits of $n$-tuples $\tau$. Thus if the orbit $\mathcal{O}$ of $\tau$ has isotropy $\mathfrak{V}$ we find

$$
K_{\infty}^{\mathcal{O}}=\bigoplus_{\tau \in \mathcal{O}} R\left[\frac{1}{\prod_{i \in \tau} g_{i}}\right]=\mathfrak{W} \otimes_{\mathfrak{V}} R\left[\frac{1}{\prod_{g \in \tau} g}\right]
$$

Finally, we argue that we can insert the group ring $\mathbb{Q}[W]$. Indeed, we are considering the map

$$
v_{E \supset F}: \mathbb{R}_{a}(E)^{(\mathbb{W} G)_{E}^{e}} \otimes_{\mathbb{R}_{a}(F)^{(\mathbb{W} G)_{F}^{e}}} N(F)^{(\mathbb{W} G)_{F}^{e}} \longrightarrow\left[\mathbb{R}_{a}(E) \otimes_{\mathbb{R}_{a}(F)} N(F)\right]^{(\mathbb{W} G)_{E}^{e}}
$$

We have observed that if $v_{E \supset F}$ is an isomorphism for $N(F)=\mathbb{R}_{a}(F)$ then it is also an isomorphism when $N(F)$ comes from $f_{(K)}^{\mathbb{N}}(I)$ with $I=H_{*}\left(B \mathbb{T} / K^{L \mathbb{T} / K}\right)$. We now show that, similarly, if $v_{E \supset F}$ is an isomorphism for $N(F)=\mathbb{R}_{a}(F)[W]$ then it is also an isomorphism when $N(F)$ comes from $f_{(K)}^{\mathbb{N}}(I[W])$. For the case when $N(F)=\mathbb{R}_{a}(F)[W]$ let us note that $N_{G}(E) \subseteq N_{G}(F)$; this gives a map of Weyl groups $W_{G}(E) \rightarrow W_{G}(F)$, and passing to quotients under their respective maximal tori, we have an inclusion $W_{G}(E) /\left(\mathbb{T} / K_{s}\right) \subseteq W_{G}(F) /\left(\mathbb{T} / L_{t}\right)$ of coset spaces.

Now for any connected Lie group $\Gamma$ with maximal torus $T$, the rational Serre spectral sequence of $\Gamma / T \rightarrow B T \rightarrow B \Gamma$ collapses to give an isomorphism

$$
H^{*}(B T) \cong H^{*}(B \Gamma) \otimes H^{*}(\Gamma / T)
$$

of $H^{*}(B \Gamma)[W]$-modules. Furthermore the Weyl group acts trivially on the first factor. For example

$$
H^{*}\left(B \mathbb{T} / K_{s}\right)=H^{*}\left(B W_{G}^{e} E\right) \otimes H^{*}\left(N_{G}^{e}(E) / \mathbb{T}\right),
$$

so that when we invert $\mathcal{E}_{K_{0} / K_{S}}$ we find

$$
\mathbb{R}_{a}(E)=\left(\Psi \mathbb{R}_{a}\right)(E) \otimes H^{*}\left(N_{G}^{e}(E) / \mathbb{T}\right) .
$$

Using this we may identify $v_{E \supseteq F}$ as

$$
\begin{aligned}
\Psi \mathbb{R}_{a}(E) \otimes \Psi \mathbb{R}_{a}(F)\left[\Psi \mathbb{R}_{a}(F) \otimes H^{*}\left(N_{G}^{e}(F) / \mathbb{T}\right)[W]\right]^{(\mathbb{W} G)_{F}^{e}} \\
\stackrel{\nu_{E \supset F}}{\longrightarrow}\left[\mathbb{R}_{a}(E) \mathbb{R}_{a}(F) \mathbb{R}_{a}(F)[W]\right]^{(\mathbb{W} G)_{E}^{e}} \\
=\left[\mathbb{R}_{a}(E)[W]\right]^{(\mathbb{W} G)_{E}^{e}} \\
=\left(\Psi \mathbb{R}_{a}\right)(E) \otimes\left[H^{*}\left(N_{G}^{e}(E) / \mathbb{T}\right)[W]\right]^{(\mathbb{W} G)_{E}^{e}} .
\end{aligned}
$$

This compares two free $\Psi \mathbb{R}_{a}(E)$-modules obtained by tensoring with the vector spaces

$$
\left[H^{*}\left(N_{G}^{e}(F) / \mathbb{T}\right)[W]\right]^{(\mathbb{W} G)_{F}^{e}} \text { and }\left[H^{*}\left(N_{G}^{e}(E) / \mathbb{T}\right)[W]\right]^{(\mathbb{W} G)_{E}^{e}}
$$


We note that they are both vector spaces of dimension $|W|$ (they are not isomorphic as graded vector spaces, but $E \neq F$ so $\mathbb{R}_{a}(E)$ is 2-periodic and tensoring gives abstractly isomorphic $\Psi \mathbb{R}_{a}(E)$-modules).

Finally, we observe that $v$ is obtained from a $\mathbb{W} G_{E}$-equivariant $\mathbb{R}_{a}(E)$-module map

$$
v_{E \supset F}: \mathbb{R}_{a}(E) \otimes_{\mathbb{R}_{a}(F)^{(\mathbb{W} G)_{F}^{e}}}\left[\mathbb{R}_{a}(F)[W]\right]^{(\mathbb{W} G)_{F}^{e}} \longrightarrow \mathbb{R}_{a}(E) \otimes_{\mathbb{R}_{a}(F)} \mathbb{R}_{a}(F)[W]
$$

by passage to $\mathbb{W} G_{E}^{e}$-fixed points. This map is surjective since $\mathbb{R}_{a}(E)[W]$ is generated as an $\mathbb{R}_{a}(E)\left[\mathbb{W} G_{E}\right]$-module by $\left(\mathbb{R}_{a}(F)[W]\right)^{\mathbb{W} G_{F}^{e}}$. Hence $v$ is an isomorphism as required.

\section{D Toral descent from $G$ to $\mathbb{N}$}

The descent property now follows from the result for arbitrary modules.

Corollary 7.11 We have an adjunction

$$
\theta_{*}: \mathcal{A}(G, \text { toral }) \rightleftarrows \mathcal{A}(\mathbb{T})[\mathbb{W} G]: \Psi,
$$

for which the unit is an isomorphism.

Proof In the light of Lemmas 7.1 and 7.6, this is immediate from Proposition 5.9.

\section{Homological algebra of $\mathcal{A}(G$, toral $)$}

In this section we deduce from known properties of $\mathcal{A}(\mathbb{T})$ the facts we need about the homological algebra from $\mathcal{A}(G$, toral). In particular, we show it has finite injective dimension equal to the rank.

\section{A Right adjoints to evaluation}

The study of $\mathcal{A}(\mathbb{T})$ in [11] shows that $\mathcal{A}(\mathbb{T})$ has sufficiently many injectives. Indeed, it is shown that enough injectives can be imported from module categories using right adjoints $f_{K}^{\mathbb{T}}$ to evaluation at subgroups $K$. We will not repeat the argument here in detail, but the idea is to argue by induction on the supporting codimension

$$
\operatorname{scd}(M):=\min \{\operatorname{dim}(\mathbb{T} / K) \mid M(K) \neq 0\}
$$

of a nonzero module $M$. One may find a map from any module $M \neq 0$ to a sum of injectives $f_{K}^{\mathbb{T}}(I)$ which is a monomorphism at subgroups of codimension $\operatorname{scd}(M)$. 
The general case can be built up from this. Accordingly, it suffices here to discuss the right adjoints to evaluation.

The starting point from [11] is that for any closed subgroup $K \subseteq \mathbb{T}$ of codimension $c$, there is a right adjoint $f_{K}^{\mathbb{T}}$ to evaluation at $K$ :

$$
\text { eval }_{K}: \mathcal{A}(\mathbb{T})_{\mathrm{scd} \geq c} \rightleftarrows \text { torsion- } H^{*}(B \mathbb{T} / K) \text {-modules }: f_{K}^{\mathbb{T}}
$$

We may combine these phenomena over a $\mathbb{W} G$-orbit $(K)$. The point is that the distinct subgroups $K_{i}$ in the orbit are of the same codimension in $\mathbb{T}$ and hence only cotorally related if they are equal:

$$
\operatorname{eval}_{(K)}: \mathcal{A}(\mathbb{T})_{\operatorname{scd} \geq c} \rightleftarrows \prod_{K^{\prime} \in(K)} \text { torsion- } H^{*}\left(B \mathbb{T} / K^{\prime}\right)-\text { modules }: f_{(K)}^{\mathbb{T}} \text {. }
$$

This is compatible with the $\mathbb{W} G$-action. To describe the structure, note that we have an inclusion $(K) \rightarrow \Sigma_{a}(\mathbb{T})$ of posets with $\mathbb{W} G$-action. Because $(K)$ is a discrete poset it is reasonable to write $H^{*}(B \mathbb{T} /(K))$ for the restriction of $\mathbb{R}_{a}$ to $(K)$. Since $(K)$ is a transitive $\mathbb{W} G$-set, there is an equivalence

$$
H^{*}(B \mathbb{T} /(K))[\mathbb{W} G] \text {-modules } \simeq H^{*}(B \mathbb{T} / K)\left[(\mathbb{W} G)_{K}\right] \text {-modules. }
$$

Thus we have an adjunction

$$
\operatorname{eval}_{(K)}: \mathcal{A}(\mathbb{T})[\mathbb{W} G]_{\mathrm{scd} \geq c} \rightleftarrows \text { torsion- } H^{*}(B \mathbb{T} /(K))[\mathbb{W} G]-\text { modules }: f_{(K)}^{\mathbb{N}}
$$

We will generally specify the particular subgroup $K$ and take the argument of $f_{(K)}^{\mathbb{N}}$ to be an $H^{*}(B \mathbb{T} / K)\left[(\mathbb{W} G)_{K}\right]$-module. The right adjoint to evaluation on $\mathcal{A}(G$, toral $)$ can now be defined in terms of the functor for $\mathbb{N}$.

Lemma 8.1 The right adjoint to evaluation at $K$ is given by the formula

$$
f_{(K)}^{G}(M)=\Psi f_{(K)}^{\mathbb{N}}\left(\theta_{*} M\right),
$$

where $M$ is an $H^{*}\left(B W_{G}^{e} K\right)\left[W_{G}^{d} K\right]$-module. We have the commutative diagram:

$$
\begin{aligned}
& \mathcal{A}(\mathbb{T})[\mathbb{W} G]_{\mathrm{scd} \geq c} \stackrel{f_{(K)}^{\mathbb{N}}}{\longleftarrow} \text { torsion- } H^{*}(B \mathbb{T} / K)\left[(\mathbb{W} G)_{K}\right] \text {-modules } \\
& \Psi \downarrow \downarrow \Psi^{\mathbb{W} W_{G}^{e} K} \\
& \mathcal{A}(G, \text { toral })_{\mathrm{scd} \geq c} \overleftarrow{f_{(K)}^{G}} \text { torsion- } H^{*}\left(B W_{G}^{e} K\right)\left[W_{G}^{d}(K)\right] \text {-modules }
\end{aligned}
$$


Proof We make the calculation

$$
\begin{aligned}
& \operatorname{Hom}_{\mathcal{A}(G, \text { toral })}\left(X, f_{(K)}^{G}(M)\right) \\
& =\operatorname{Hom}_{\mathcal{A}(G, \text { toral })}\left(X, \Psi f_{(K)}^{\mathbb{N}}\left(\theta_{*} M\right)\right) \\
& =\operatorname{Hom}_{\mathcal{A}(\mathbb{T})[\mathbb{W} G]}\left(\theta_{*} X, f_{(K)}^{\mathbb{N}}\left(\theta_{*} M\right)\right) \\
& =\operatorname{Hom}_{H *(B \mathbb{T} / K)\left[\mathbb{W} G_{K}\right]}\left(\left(\theta_{*} X\right)(K), \theta_{*} M\right) \\
& =\operatorname{Hom}_{H^{*}(B \mathbb{T} / K)}\left(H^{*}(B \mathbb{T} / K) \otimes_{H^{*}\left(B W_{G}^{e} K\right)} X(K), \theta_{*} M\right)^{\mathbb{W} G_{K}} \\
& =\operatorname{Hom}_{H^{*}\left(B W_{G}^{e} K\right)}\left(X(K), H^{*}(B \mathbb{T} / K) \otimes_{H^{*}\left(B W_{G}^{e} K\right)} M\right)^{\mathbb{W} G_{K}} \\
& =\operatorname{Hom}_{H^{*}\left(B W_{G}^{K} K\right)}(X(K), M)^{W_{G}^{d} K} \text {. }
\end{aligned}
$$

\section{B The category $\mathcal{A}(\mathbb{N}$, toral)}

The evaluation functors immediately bring $\mathcal{A}(\mathbb{N}$, toral) under control.

Lemma 8.2 The abelian category $\mathcal{A}(\mathbb{N}$, toral $)=\mathcal{A}(\mathbb{T})[\mathbb{W}]$ has enough injectives and is of injective dimension equal to the rank.

Proof In the category of $H^{*}(B \mathbb{T} / K)\left[(\mathbb{W} G)_{K}\right]$-modules, any torsion injective embeds in

$$
\operatorname{Hom}_{\mathbb{Q}}\left(\mathbb{Q}\left[(\mathbb{W} G)_{K}\right], H_{*}(B \mathbb{T} / K)\right)=\left(H^{*}(B \mathbb{T} / K)\left[(\mathbb{W} G)_{K}\right]\right)^{\vee} .
$$

Applying $f_{(K)}^{\mathbb{N}}$ we obtain enough injectives in $\mathcal{A}(\mathbb{T})[\mathbb{W} G]$.

Since

$$
\operatorname{Hom}_{\mathcal{A}(\mathbb{T})[\mathbb{W} G]}(M, N)=\operatorname{Hom}_{\mathcal{A}(\mathbb{T})}(M, N)^{\mathbb{W} G}
$$

and passage to fixed points is exact, it follows that the injective dimension of $\mathcal{A}(\mathbb{T})[\mathbb{W} G]$ is no more than that of $\mathcal{A}(\mathbb{T})$. The case of coinduced modules shows they are equal.

\section{C The category $\mathcal{A}(G$, toral $)$}

The properties we want for $\mathcal{A}(G$, toral) itself can now be deduced formally from what we have proved for $\mathcal{A}(\mathbb{N}$, toral $)$.

Proposition 8.3 The abelian category $\mathcal{A}(G$, toral) has enough injectives and is of injective dimension equal to the rank of $G$. 
Proof Since we are working over the rationals, $H^{*}(B \mathbb{T} / K)$ is free over $H^{*}\left(B W_{G}^{e} K\right)$ and $\theta_{*}$ is exact. The right adjoint $\Psi$ therefore preserves injectives, and $\Psi I$ is injective in $\mathcal{A}(G$, toral) for every injective $I$ in $\mathcal{A}(\mathbb{T})[\mathbb{W} G]$. Consequently, if we apply $\Psi$ to an injective resolution of $M$ we obtain an injective resolution of $\Psi M$. Since the unit of the adjunction is an isomorphism (Proposition 5.9 and Corollary 7.11), all objects of $\mathcal{A}(G$, toral $)$ are in the image of $\Psi$ and there are enough injectives in $\mathcal{A}(G$, toral).

Since $\mathcal{A}(\mathbb{T})$ is of injective dimension $r$ [12], it follows that $\mathcal{A}(G$, toral) is of injective dimension $\leq r$. To see that this bound is achieved, we may consider free spectra (which is to say torsion modules over the polynomial ring $H^{*}\left(B G_{e}\right)$ on $r$ generators), or more specifically $G_{+}$(which is to say the torsion module $\mathbb{Q}\left[G_{d}\right]$ ).

\section{Part II Topology}

\section{Toral detection}

We show that the toral part of $G$-spectra is detected in $\mathbb{T}$-equivariant homotopy. This is the key result that makes this entire approach viable.

\section{A Idempotents}

Underlying the structure of any monoidal category is the endomorphism ring of the unit object, which in our case is the ring of stable homotopy groups of $S^{0}$. Accordingly, we recall how the Burnside ring $A(G)=\left[S^{0}, S^{0}\right]^{G}$ is related to spaces of subgroups. Given a stable map $f: S^{0} \rightarrow S^{0}$, the degree of geometric fixed points defines a function $\operatorname{deg}(f): \mathcal{F}(G) \rightarrow \mathbb{Z}$ from the set $\mathcal{F}(G)$ of subgroups of $G$ with finite index in their normalizers. It is clearly constant on conjugacy classes, and one may show that $\operatorname{deg}(f)$ is continuous in the Hausdorff metric topology. It was shown by tom Dieck [5] that the map

$$
A(G) \longrightarrow C_{G}(\mathcal{F}(G), \mathbb{Z})
$$

is injective and that it is a rational isomorphism. Furthermore $C_{G}(\mathcal{F}(G), \mathbb{Z}) \otimes \mathbb{Q}$ is isomorphic to $C_{G}(\mathcal{F}(G), \mathbb{Q})$. Finally, it is easy to deduce the degree of the geometric fixed points under any subgroup: if $K$ is not of finite index in its normalizer then $\operatorname{deg}\left(f^{K}\right)=\operatorname{deg}\left(f^{H}\right)$ whenever $K$ is cotoral in $H$.

Next we note that the conjugacy class of maximal tori is open and closed in $\mathcal{F}(G)$, so there is an idempotent $e_{\mathbb{T}} \in A(G)$ with support on $(\mathbb{T})$ and the degree of its $K$-fixed points is 1 for subgroups of a maximal torus and 0 otherwise. 
We may then localize with respect to $e_{\mathbb{T}} S^{0}$, and obtain

$$
\text { toral- } G-\text { spectra }=e_{\mathbb{T}}[G-\text { spectra }] .
$$

Lemma 9.1 Writing $\Lambda(\mathbb{T})$ for the family of subgroups of some maximal torus, the natural map $E \Lambda(\mathbb{T})_{+} \rightarrow S^{0}$ induces an equivalence $E \Lambda(\mathbb{T})_{+} \simeq e_{\mathbb{T}} S^{0}$.

Proof By definition the $K$-fixed point space of $E \Lambda(\mathbb{T})_{+}$is equivalent to $S^{0}$ if $K$ lies in a maximal torus and is a point otherwise. The map is therefore an equivalence in geometric $K$-fixed points for all $K$ and hence a weak equivalence.

Corollary 9.2 We have

$$
\left[E \Lambda(\mathbb{T})_{+}, E \Lambda(\mathbb{T})_{+}\right]^{G}=\left[S^{0}, S^{0}\right]^{\mathbb{T}}=\mathbb{Q},
$$

which is detected by the degree in homotopy of geometric $\mathbb{T}$-fixed points.

Proof After Lemma 9.1, we see

$$
\left[E \Lambda(\mathbb{T})_{+}, E \Lambda(\mathbb{T})_{+}\right]^{G}=\left[E \Lambda(\mathbb{T})_{+}, S^{0}\right]^{G}=\left[e_{\mathbb{T}} S^{0}, S^{0}\right]^{G}=e_{\mathbb{T}} A(G)=\mathbb{Q}
$$

\section{B Toral restriction is faithful}

The key to our strategy is that the restriction from $G$ to a maximal torus $\mathbb{T}$ is faithful on toral spectra.

Proposition 9.3 The forgetful map

$$
[X, Y]^{G} \longrightarrow[X, Y]^{\mathbb{T}}
$$

is rationally split injective if $X$ is a $\Lambda(\mathbb{T})$-spectrum.

Proof Under the natural equivalence $\left[G / \mathbb{T}_{+} \wedge X, Y\right]^{G}=[X, Y]^{\mathbb{T}}$ the forgetful map corresponds to the projection $\pi: G / \mathbb{T} \rightarrow *$.

Since $X$ is a $\Lambda(\mathbb{T})$-spectrum, it is equivalent to $X \wedge E \Lambda(\mathbb{T})_{+}$, so that a splitting can be obtained from a factorization:

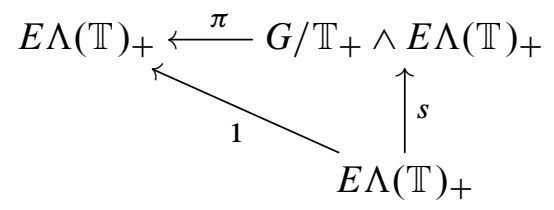

It remains to choose a suitable $s$, and we note that Corollary 9.2 shows we need only show $s$ is nontrivial in $\mathbb{T}$-geometric fixed points. In fact we will show that maps in this pattern are determined by $\pi_{0}$ of $\mathbb{T}$-geometric fixed points. 
Lemma 9.4 For each of the three pairs of spaces $X, Y$ in the above diagram we have an isomorphism

$$
[X, Y]^{G} \cong \operatorname{Hom}_{\mathbb{Q} W}\left(\pi_{0}\left(\Phi^{T} X\right), \pi_{0}\left(\Phi^{T} Y\right)\right)
$$

Proof This is already done for the edges labelled 1 or $\pi$, so we only need to deal with the edge labelled $s$ where $X=E \Lambda(\mathbb{T})_{+}$and $Y=G / \mathbb{T}_{+} \wedge E \Lambda(\mathbb{T})_{+}$.

Write $L$ for the representation of $\mathbb{T}$ given by the tangent space to $G / \mathbb{T}$ at $e \mathbb{T}$, and note the fact that $\mathbb{T}$ is a maximal abelian connected subgroup shows that $L^{\mathbb{T}}=0$. The Wirthmüller adjunction gives isomorphisms

$$
\begin{aligned}
{\left[E \Lambda(\mathbb{T})_{+}, G / T_{+} \wedge E \Lambda(\mathbb{T})_{+}\right]^{G} } & =\left[E \Lambda(\mathbb{T})_{+}, F_{T}\left(G_{+}, S^{L} \wedge E \Lambda(\mathbb{T})_{+}\right)\right]^{G} \\
& =\left[E \Lambda(\mathbb{T})_{+}, S^{L} \wedge E \Lambda(\mathbb{T})_{+}\right]^{\mathbb{T}}=\left[S^{0}, S^{L}\right]^{\mathbb{T}}=\mathbb{Q}
\end{aligned}
$$

The last isomorphism follows from the Segal-tom Dieck splitting, since the only subgroup $K$ of $\mathbb{T}$ with finite index in its normalizer is $\mathbb{T}$ itself. Following through the adjunctions, the composite isomorphism is given by forgetting from $G$ to $\mathbb{T}$ and composing with the $\mathbb{T}$-map

$$
G / T_{+} \wedge E \Lambda(\mathbb{T})_{+} \longrightarrow S^{L} \wedge E \Lambda(\mathbb{T})_{+}
$$

induced by the Pontrjagin-Thom map $G / T \rightarrow S^{L}$. It follows that maps are detected by degree in $\mathbb{T}$-geometric fixed points.

According to Lemma 9.4, we need only consider

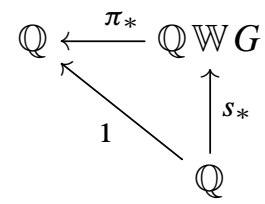

and select $s$ so that $|\mathbb{W} G| s_{*}$ is the norm map.

Remark 9.5 To be more specific, we can take $|\mathbb{W} G| s$ to be the composite $E \Lambda(\mathbb{T})_{+} \rightarrow F_{\mathbb{T}}\left(G_{+}, E \Lambda(\mathbb{T})_{+}\right) \stackrel{F_{\mathbb{T}}\left(G_{+}, i_{L}\right)}{\longrightarrow} F_{\mathbb{T}}\left(G_{+}, S^{L} \wedge E \Lambda(\mathbb{T})_{+}\right) \simeq G / \mathbb{T}_{+} \wedge E \Lambda(\mathbb{T})_{+}$ where the first map is the adjunct of the identity and the last is the standard Wirthmüller equivalence. 


\section{Borel cohomology and the associated homology theory}

\section{A Classical isomorphisms}

The essential ingredients in the proof that $\mathcal{A}(G$, toral $)$ provides an effective invariant are classical facts about the cohomology of the Borel construction. We will need to apply the results to $W_{G}^{e} K$ for various subgroups $K$ of $G$, so in this section we take $\Gamma$ to be a compact Lie group with maximal torus $\mathbb{T}$, torus-normalizer $\mathbb{N}=N_{\Gamma} \mathbb{T}$ and Weyl group $\mathbb{W} \Gamma=N_{\Gamma} \mathbb{T} / \mathbb{T}$.

Lemma 10.1 If $Z$ is a free $\Gamma$-space then we have natural isomorphisms

(i) $H^{*}(Z / \mathbb{N}) \cong H^{*}(Z / T)^{\mathbb{W} \Gamma,}$

(ii) $H^{*}(Z / \Gamma) \cong H^{*}(Z / \mathbb{N})$,

(iii) $H^{*}(Z / \Gamma) \cong H^{*}(Z / T)^{\mathbb{W} \Gamma}$; and

(iv) if $\Gamma$ is connected, there is a natural isomorphism

$$
H^{*}(B T) \otimes_{H^{*}(B \Gamma)} H^{*}(Z / \Gamma) \stackrel{\cong}{\leftrightarrows} H^{*}(Z / T) .
$$

Proof It suffices to treat the unbased case.

Part (i) follows since the Serre spectral sequence $Z / T \rightarrow Z / \mathbb{N} \rightarrow B \mathbb{W} \Gamma$ collapses when the group order is invertible.

Part (ii) follows from the Serre spectral sequence of $\Gamma / \mathbb{N} \rightarrow X / \mathbb{N} \rightarrow X / \Gamma$, since $\Gamma / \mathbb{N}$ is rationally contractible.

Part (iii) follows by combining parts (i) and (ii).

Part (iv) follows from the Eilenberg-Moore spectral sequence of the pullback square:

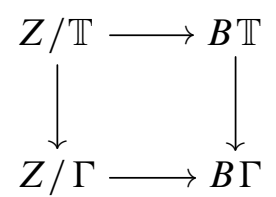

We note that connectedness of $\Gamma$ ensures $B \Gamma$ is 1 -connected, and working over $\mathbb{Q}$ ensures that $H^{*}(B \mathbb{T})$ is free over $H^{*}(B \Gamma)$.

Corollary 10.2 For any $\mathbb{N}$-spectrum $B$, the map $i: B \rightarrow \Gamma_{+} \wedge_{\mathbb{N}} B$ induces an isomorphism in $H_{\mathbb{N}}^{*}$. 
Proof It suffices to prove the case when $B$ is the suspension spectrum of $Z_{+}$for an unbased space $Z$. It is convenient to view this as the $\Gamma$ Borel cohomology of

$$
\Gamma \times_{\mathbb{N}} Z \longrightarrow \Gamma \times_{\mathbb{N}} \Gamma \times_{\mathbb{N}} Z \cong \Gamma / \mathbb{N} \times \Gamma \times_{\mathbb{N}} Z
$$

Since the composite with projection is the identity, it therefore suffices to observe that by the lemma, $\Gamma / \mathbb{N} \rightarrow *$ induces an isomorphism.

\section{B Fixed points and induced spaces}

The purpose of this subsection is to show that the $L$-fixed point spaces of induced spaces are made up of copies of induced spaces of Weyl groups.

More precisely, we suppose $L \subseteq \mathbb{T}$ and consider its conjugates inside $\mathbb{T}$, which consists of the $\mathbb{W} G$-orbit of $L$, and we suppose the groups are $L=L_{1}, L_{2}, \ldots, L_{c}$ with $L_{i}=L^{\gamma_{i}}$. In the usual way if $A$ is a $\mathbb{T}$-space then $A^{L_{i}}=\gamma_{i}^{-1}\left(A^{L}\right)$.

Lemma 10.3 For a $\mathbb{T}$-space $A$ we have

$$
\left(G \times_{\mathbb{T}} A\right)^{L}=\coprod_{i} W_{G}(L) \gamma_{i} \times_{\mathbb{T} / L_{i}} A^{L_{i}}
$$

Proof We note that the condition for $[g, a]$ to be $L$-fixed is that for each $l \in L$ there is a $t \in \mathbb{T}$ so that $l g=g t$ and $t^{-1} a=a$. The first condition determines $t$, so $[g, a]$ is only fixed if $L^{g} \subseteq \mathbb{T}$ and then $a$ is fixed by $L^{g}$. Thus we obtain

$$
\coprod_{i} N_{G}(L) \gamma_{i} \times_{\mathbb{T}} A^{L_{i}} \longrightarrow \coprod_{i} N_{G}(L) / L \gamma_{i} \times \mathbb{T} / L_{i} A^{L_{i}}
$$

as claimed.

Corollary 10.4 For any $T$-space $A$, the map $\mathbb{N} \times_{\mathbb{T}} A \rightarrow G \times_{\mathbb{T}} A$ induces an isomorphism of $W_{G} L$-equivariant Borel cohomology of $L$-fixed points.

Proof From Lemma 10.3, we see that the map is a disjoint union of instances of

$$
W_{\mathbb{N}}(L) \times_{\mathbb{T} / L} A^{L} \longrightarrow W_{G}(L) \times_{\mathbb{T} / L} A^{L} .
$$

This in turn is an instance of Corollary 10.2 with $\Gamma=W_{G} L$. 


\section{C Adjoint representations}

It is extremely interesting to see how the adjoint representation behaves in moving between $G$ and $\mathbb{N}$. Alternatively stated, this amounts to understanding the adjoint representation in the Adams isomorphism. We write $L G$ for the adjoint representation, which is to say the tangent space at the identity of $G$ with $G$ acting by conjugation. For the torus $\mathbb{T}$ there is also a rational version $L_{\mathbb{Q}} \mathbb{T}=H_{1}(\mathbb{T} ; \mathbb{Q})$, so that there is a natural isomorphism $L_{\mathbb{Q}} \mathbb{T} \otimes \mathbb{R} \cong L \mathbb{T}$.

Lemma 10.5 We have a natural isomorphism $H_{*}^{G}\left(X \wedge S^{L G}\right)=H_{*}^{\mathbb{N}}\left(X \wedge S^{L \mathbb{T}}\right)$.

Proof by stable equivariant formalism If $X$ is a finite free $G$-space then we have natural isomorphisms

$$
\begin{aligned}
H_{*}^{G}\left(\Sigma^{L G} X\right) & \cong\left[S^{0}, X \wedge H\right]_{*}^{G} \\
& \cong[D X, H]_{*}^{G} \\
& \cong H_{G}^{*}(D X) \\
& \cong H_{N}^{*}(D X) \\
& \cong[D X, H]_{*}^{N} \\
& \cong\left[S^{0}, X \wedge H\right]_{*}^{\mathbb{N}} \\
& \cong H_{*}^{\mathbb{N}}\left(\Sigma^{L \mathbb{T}} X\right) .
\end{aligned}
$$

The two equivalences changing $X$ to $D X$ come from the formal properties of duality. Since $X$ is finite and free, $D X$ is free, giving the isomorphisms with Borel cohomology. The one relating $G$-equivariant and $N$-equivariant Borel cohomology is Lemma 10.1(ii). The first and last isomorphisms are instances of the Adams isomorphism.

Proof by Lie group theory We observe directly that $S^{L T} \rightarrow S^{L G}$ induces an isomorphism in $H_{\mathbb{N}}^{*}$, which is to say that multiplication by the Euler class of $L G / L T$ is an isomorphism. More precisely, if $g=\operatorname{dim} G$ and $t=\operatorname{dim} \mathbb{T}$, we show that the horizontals in the following diagram are isomorphisms of the $\mathbb{W} G$-invariants

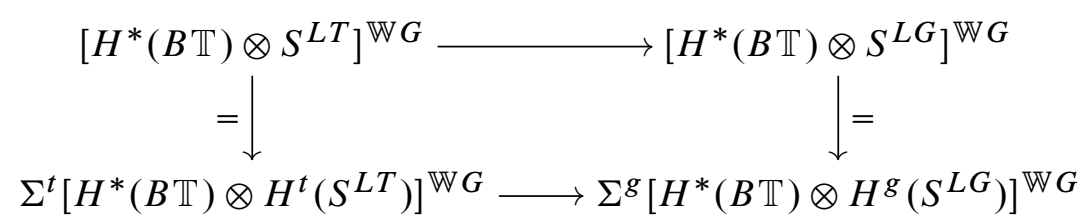

given by the multiplication by the product of the Euler classes of the positive roots. 
We adjoin exterior variables to give a context, writing $A(V)=E(\Sigma V) \otimes P\left(\Sigma^{2} V\right)$ for a vector space $V$. For an element $v \in V$ we write $\lambda(v)$ for the corresponding element of $\Sigma V$ and $c(v)$ for the element of $\Sigma^{2} V$. We consider the special case $V=L_{\mathbb{Q}} \mathbb{T}$, so that $H^{*}(B \mathbb{T})=P\left(\Sigma^{2} V\right)$. Thus

$$
H_{\mathbb{T}}^{*}\left(S^{L T}\right) \subseteq A(V)
$$

consists of the $H^{*}(B \mathbb{T})$-submodule generated by $\operatorname{det}(\Sigma V)$. Choosing an ordered basis $e_{1}, \ldots, e_{r}$ of $V$ we may let $\delta=\lambda\left(e_{1}\right) \wedge \cdots \wedge \lambda\left(e_{r}\right)$ be a generator of $\operatorname{det}(\Sigma V)$. Now consider the adjoint representation of $G$ and choose a set $R_{+}$of positive roots. If we take $\kappa=\prod_{\alpha \in R_{+}} c(\alpha)$ then $\delta \kappa$ is the Euler class of $L G$.

The result is now Solomon's lemma [18], but perhaps it is illuminating to sketch the proof in this case. We observe that $\delta \kappa$ is $\mathbb{W} G$ invariant. Indeed, associated to $R_{+}$ there is the Weyl chamber on which the roots are positive and $\mathbb{W} G$ is generated by reflections $s_{\alpha}$ in the walls of the Weyl chamber. Since $s_{\alpha}$ is a reflection $s_{\alpha} \delta=-\delta$. On the other hand $s_{\alpha}$ negates $\alpha$ and permutes the other positive roots [4, 4.10]. Hence $s_{\alpha}$ fixes $\delta \kappa$.

Since $H^{*}(B G)=H^{*}(B \mathbb{T})^{\mathbb{W} G}$ it follows that

$$
H_{G}^{*}\left(S^{L G}\right)=H^{*}(B G) \cdot \delta \kappa \subseteq H_{\mathbb{T}}^{*}\left(S^{L \mathbb{T}}\right)^{\mathbb{W} G} .
$$

Now we argue that any element $\delta f$ of the invariants is divisible by each $c(\alpha)$. Since $\operatorname{det}\left(s_{\alpha}\right)=-1$, we find $f\left(s_{\alpha} c(v)\right)=-f(c(v))$ for each $v$. Accordingly, for each $v$ in the reflecting hyperplane $f(c(v))=0$. If we choose a basis consisting of $\alpha$ together with elements of the reflecting hyperplane we see $c(\alpha)$ divides $f$. Since any pair of positive roots are linearly independent, it follows that $f$ is divisible by $\kappa$ as required.

\section{D The dual of Borel cohomology}

We let $b$ denote the representing $G$-spectrum for Borel cohomology, so that, by definition,

$$
b_{G}^{*}(X)=H^{*}\left(E G_{+} \wedge_{G} X\right)=\left[E G_{+} \wedge X, H\right]_{G}^{*}=\left[X, F\left(E G_{+}, H\right)\right]_{G}^{*} .
$$

This shows the representing spectrum is given by

$$
b=F\left(E G_{+}, H\right) .
$$

The associated homology theory is defined by

$$
b_{*}^{G}(X)=\left[S^{0}, X \wedge b\right]_{*}^{G} .
$$


The canonical warning is that this is not homology of the Borel construction. Instead, we have

$$
b_{*}^{G}(X)=\lim _{\alpha} b_{*}^{G}\left(X_{\alpha}\right)
$$

where $X$ is the directed colimit of finite subspectra $X_{\alpha}$. For finite spectra $Y$ we have

$$
b_{*}^{G}(Y)=\left[S^{0}, Y \wedge b\right]_{*}^{G}=[D Y, b]_{G}^{*}=b_{G}^{*}(D Y)=H^{*}\left(E G_{+} \wedge_{G} D Y\right) .
$$

Remark 10.6 This calculation can be viewed as one of the motivations for BorelMoore homology, according to which $b_{*}^{G}(X)$ would be the Borel-Moore homology associated to Borel cohomology. However, since the essence of Borel-Moore homology is really the use of locally finite chains it would be misleading to call this Borel BorelMoore homology.

We will need a standard observation.

Lemma 10.7 For finite $G$-spectra $Y$ we have $b \wedge Y \simeq *$ if and only if $b \wedge D Y \simeq *$.

Proof Since $b$ is a ring $G$-spectrum it follows that if $b \wedge Y \simeq *$ then $F(Y, b) \simeq *$.

Our main use of this homology theory is to formulate appropriate analogues of Lemma 10.1.

Lemma 10.8 Suppose $\Gamma$ is a compact Lie group with maximal torus $T$ and Weyl group $\mathbb{W} \Gamma$ and that the order of $\mathbb{W} \Gamma$ is invertible in the coefficients. For $\Gamma$-spectra $A$, there is a natural isomorphism

$$
b_{*}^{\Gamma}(A)=\left[b_{*}^{T}(A)\right]^{\mathbb{W} \Gamma} .
$$

Proof The forgetful map

$$
\left[S^{0}, b \wedge A\right]^{\Gamma} \longrightarrow\left[S^{0}, b \wedge A\right]^{T}
$$

supplies a natural transformation

$$
b_{*}^{\Gamma}(A) \longrightarrow\left[b_{*}^{T}(A)\right]^{\mathbb{W} \Gamma} .
$$

Since the order of $\mathbb{W} \Gamma$ is invertible, both terms are homology theories, and both preserve filtered colimits. The transformation is an isomorphism for finite complexes by Lemma 10.1(iii). 


\section{The functor from $G-$ spectra to $\mathcal{A}(G$, toral $)$}

We have built a model of toral $G$-spectra by comparison with the model for $\mathbb{T}$-spectra. In this section, we elucidate the relationship between these two models and thereby construct the functor $\pi_{*}^{\mathcal{A}(G)}$ from $G$-spectra to $\mathcal{A}(G$, toral).

\section{A Equivariance}

We have seen that rational $\mathbb{T}$-spectra are modelled by $\mathcal{A}(\mathbb{T})$ and that there is a functor

$$
\pi_{*}^{\mathcal{A}}: \mathbb{T} \text {-spectra } \longrightarrow \mathcal{A}(\mathbb{T})
$$

defined by

$$
\pi_{*}^{\mathcal{A}}(X)(L)=\pi_{*}^{\mathbb{T} / L}\left(D E \mathbb{T} / L_{+} \wedge \Phi^{L} X\right)
$$

and for finite $X$ this is $H_{\mathbb{T} / L}^{*}\left(D \Phi^{L} X\right)$. The image of restriction from $G$-spectra to $\mathbb{T}$-spectra has additional structure. To start with, we know that $\mathcal{A}(\mathbb{T})$ admits an action of $\mathbb{W} G$.

Lemma 11.1 The image of the composite

$$
G-\text { spectra } \longrightarrow \mathbb{T} \text {-spectra } \longrightarrow \mathcal{A}(\mathbb{T})
$$

consists of $\mathbb{W} G$-equivariant modules and $\mathbb{W} G$-equivariant maps. Accordingly, we have a functor

$$
\pi_{*}^{\mathcal{A}}: G-\text { spectra } \longrightarrow \mathcal{A}(\mathbb{T})[\mathbb{W} G]=\mathcal{A}(\mathbb{N}, \text { toral }) .
$$

Proof By definition

$$
\pi_{*}^{\mathcal{A}}(X)(K \supseteq L)=\pi_{*}^{\mathbb{T} / L}\left(S^{\infty V(K / L)} \wedge D E \mathcal{F} / L_{+} \wedge \Phi^{L} X\right) .
$$

The action of $\mathbb{W} G$ is through conjugation by group elements. This gives group homomorphisms $L \rightarrow L^{w}$, and homeomorphisms between the spaces corresponding to the groups. The homeomorphisms are equivariant for the group homomorphism.

The identification $\mathcal{A}(\mathbb{N}$, toral $)=\mathcal{A}(\mathbb{T})[\mathbb{W} G]$ is given in Lemma 6.10.

\section{$11 B$ Restriction for free spectra}

In preparation for explaining how restriction from $G$-spectra to $\mathbb{N}$-spectra is modelled, we consider the inclusion $i: H \rightarrow G$ of a subgroup. We have left and right adjoints to restriction:

$$
G-\text { spectra } \underset{i^{*}}{\longleftarrow} i_{i} \longleftarrow \text {-spectra }
$$


It is helpful to think first about what happens for free spectra. We summarize the discussion from [13]. Starting in the case when $G$ and $H$ are connected, we have a map

$$
\theta=i^{*}: H^{*}(B G) \longrightarrow H^{*}(B H) .
$$

This induces restriction of scalars $\theta^{*}$ which itself has left and right adjoints:

$$
H^{*}(B H)-\bmod \underset{\theta_{!}}{\stackrel{\theta_{*}}{\longleftarrow} \theta^{*} \longrightarrow} H^{*}(B G)-\bmod .
$$

It is apparent that the two triples of adjoint functors cannot match up. It turns out that

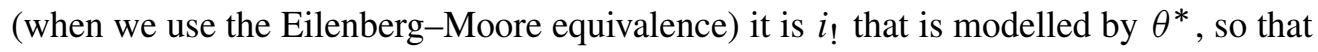
$i^{*}$ is modelled by $\theta_{*}$.

The relevant analogy for us does not involve connected groups, so we recall the general case from [13]. We write $i_{e}: H_{e} \rightarrow G_{e}$ for the inclusion of the identity component, and $i_{d}: H_{d} \rightarrow G_{d}$ for the induced map on discrete quotients (not usually injective). In algebra, we again let $\theta_{e}=i_{e}^{*}: H^{*}\left(B G_{e}\right) \rightarrow H^{*}\left(B H_{e}\right)$ for the induced map in cohomology. The main piece of data is $\theta=\left(\theta_{e}, i_{d}\right)$. It turns out that $i_{!}$is modelled by a functor we call $\theta^{*}$, which is defined on $H^{*}\left(B H_{e}\right)\left[H_{d}\right]$-modules $N$ by

$$
\theta^{*}(N)=\operatorname{Hom}_{\mathbb{Q}\left[H_{d}\right]}\left(\mathbb{Q}\left[G_{d}\right], N\right)
$$

(we note this is consistent with the previous notation when $H_{d}=G_{d}=1$ ). Restriction of groups $i^{*}$ is then modelled by the functor $\theta_{*}$ left adjoint to $\theta^{*}$, which is defined on $H^{*}\left(B G_{e}\right)\left[G_{d}\right]$-modules $M$ by

$$
\theta_{*}(M)=H^{*}\left(B H_{e}\right) \otimes_{H^{*}\left(B G_{e}\right)} M .
$$

Induction of spectra $i_{*}$ is then modelled by the functor $\theta^{\dagger}$ left adjoint to $\theta_{*}$ defined on $H^{*}\left(B H_{e}\right)\left[H_{d}\right]$-modules $N$ by

$$
\theta^{\dagger}(N)=\mathbb{Q}\left[G_{d}\right] \otimes_{\mathbb{Q}\left[H_{d}\right]} \mathbb{D}\left(G_{e} \mid H_{e}\right) \otimes_{H^{*}\left(B H_{e}\right)} N,
$$

where the relative dualizing module is defined by

$$
\mathbb{D}\left(G_{e} \mid H_{e}\right)=\operatorname{Hom}_{H^{*}\left(B G_{e}\right)}\left(H^{*}\left(B H_{e}\right), H^{*}\left(B G_{e}\right)\right) .
$$

In our case the relative dualizing module satisfies

$$
\mathbb{D}\left(G_{e} \mid H_{e}\right)=\operatorname{Hom}_{H^{*}\left(B G_{e}\right)}\left(H^{*}\left(B H_{e}\right), H^{*}\left(B G_{e}\right)\right) \simeq \Sigma^{L G / H} H^{*}\left(B H_{e}\right)
$$

and we may therefore simplify the expression for $\theta^{\dagger}$ to find

$$
\theta^{\dagger}(N)=\mathbb{Q}\left[G_{d}\right] \otimes_{\mathbb{Q}\left[H_{d}\right]} \Sigma^{L G / H} N .
$$


In the special case $H=\mathbb{N}$ we note that $H_{e}=\mathbb{T}$ and $H_{d}=\mathbb{W} G$. In view of the fact that $\mathbb{W} G / \mathbb{W}\left(G_{e}\right) \cong G_{d}$ we see that

$$
\begin{aligned}
& i_{!} \text {is modelled by } \theta^{*} N=N^{\mathbb{W} G_{e}}, \\
& i^{*} \text { is modelled by } \theta_{*} M=H^{*}(B \mathbb{T}) \otimes_{H^{*}\left(B G_{e}\right)} M \text {, } \\
& i_{*} \text { is modelled by } \theta^{\dagger} N=\left(\Sigma^{L G / \mathbb{T}} N\right)_{\mathbb{W}\left(G_{e}\right)} \text {. }
\end{aligned}
$$

\section{C The image of a spectrum in the model}

We now make explicit the functor we use to relate $G$-spectra to $\mathcal{A}(G$, toral $)$. The motivation is that restriction to the maximal torus is homotopically faithful, but the special form of the objects in the image mean that we can pass to invariants without losing information.

We will need to consider the functor

$$
\mathcal{A}(\mathbb{N}, \text { toral })=\mathcal{A}(\mathbb{T})[\mathbb{W} G] \stackrel{\Psi}{\longrightarrow} \mathcal{A}(G, \text { toral })
$$

from Proposition 5.9, where we use the Lie group component structure of Section 4E. We recall that it was shown to have left adjoint $\theta_{*}$ defined by

$$
\theta_{*}(Y)=\mathbb{R}_{a} \otimes_{\mathbb{R}_{\mathrm{inv}}} Y,
$$

which on subgroups $K \subseteq \mathbb{T}$ is

$$
\left(\theta_{*} Y\right)(K)=H^{*}(B \mathbb{T} / K) \otimes_{H^{*}\left(B W_{G}^{e} K\right)} Y(K) .
$$

Remark 11.2 In view of the isomorphism $\mathbb{Q}[\mathbb{W} G]^{\mathbb{W}} W_{G}^{e} K=\mathbb{Q}\left[W_{G}^{d} K\right]$, the relationship between the two notations is

$$
(\Psi X)(K)=X(K)^{\mathbb{W} W_{G}^{e} K}=\operatorname{Hom}_{(\mathbb{W} G)_{K}}\left(\mathbb{Q} W_{G}^{d} K, X(K)\right)=\left(\theta^{*} X\right)(K) .
$$

Definition 11.3 The functor $\pi_{*}^{\mathcal{A}(G)}: G$-spectra $\rightarrow \mathcal{A}(G$, toral $)$ is defined as the illustrated composite of three functors:

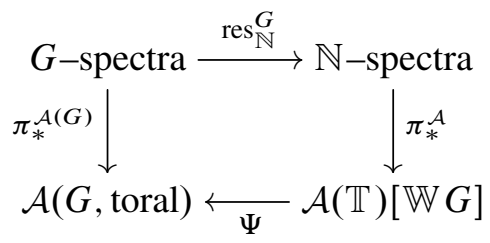

Remark 11.4 We note that specializing the definition to the case $G=\mathbb{N}$ gives $\pi_{*}^{\mathcal{A}(\mathbb{N})}=\pi_{*}^{\mathcal{A}(\mathbb{T})}=\pi_{*}^{\mathcal{A}}$, which is consistent according to Lemma 11.1. 
We immediately express $\pi_{*}^{\mathcal{A}(G)}$ more directly in terms of $G$-equivariant data.

Proposition 11.5 For any $G$-spectrum $X$, and any subgroup $K \subseteq \mathbb{T}$, we have

$$
\pi_{*}^{\mathcal{A}(G)}(X)(K)=b_{*}^{W_{G}^{e} K}\left(\Phi^{K} X\right) .
$$

If $X$ is a finite $G$-spectrum, we can express this directly in terms of Borel cohomology of fixed points of the dual

$$
\pi_{*}^{\mathcal{A}(G)}(X)(K)=H_{W_{G}^{e} K}^{*}\left(\Phi^{K}(D X)\right)
$$

Remark 11.6 It would be possible to give the statement of the proposition as the definition of $\pi_{*}^{\mathcal{A}(G)}(X)$. We used Definition 11.3 instead because the deduction of the proposition from the definition is a little more elementary than the reverse deduction. Indeed, if $\Gamma$ is a connected group ( $\operatorname{such}$ as $W_{G}^{e} K$ ) with maximal torus $T$ and $A$ is a $\Gamma$-space (which might have arisen as $\Phi^{K} D X$ in some cases), Lemma 10.1 gives the two formulae

$$
H_{\Gamma}^{*}(A)=H_{\mathbb{T}}^{*}(A)^{\mathbb{W} \Gamma}
$$

and

$$
H_{\mathbb{T}}^{*}(A)=H^{*}(B T) \otimes_{H *(B \Gamma)} H_{\Gamma}^{*}(A) .
$$

We view the first as more elementary than the second.

Proof By definition

$$
\pi_{*}^{\mathcal{A}(G)}(X)(K)=\pi_{*}^{\mathbb{T} / K}\left(D E \mathbb{T} / K_{+} \wedge \Phi^{K} X\right)=b_{*}^{\mathbb{T} / K}\left(\Phi^{K} X\right) .
$$

The result now follows by applying Lemma 10.8 with $\Gamma=W_{G}^{e} K$ and $A=\Phi^{K} X$.

\section{D Restriction}

As in the case of free spectra, it will emerge that $\theta^{*}=\Psi$ corresponds to coinduction, and its left adjoint $\theta_{*}$ corresponds to restriction.

Proposition 11.7 The following diagram commutes:

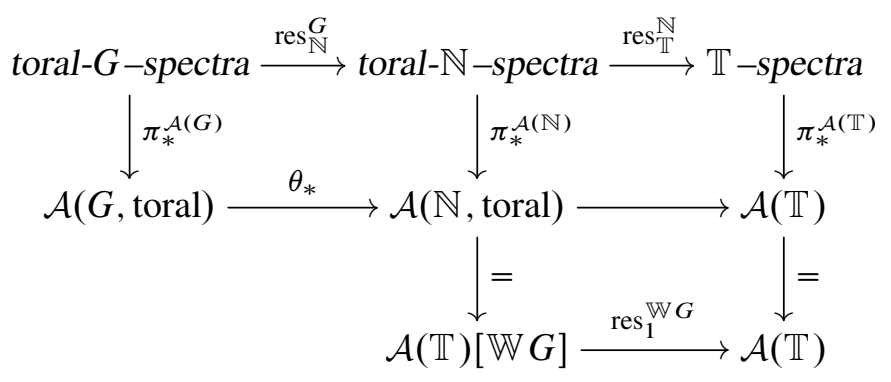


Proof The right-hand two squares commute by the definition of $\pi_{*}^{\mathcal{A}}$ together with Lemmas 11.1 and 6.10 .

By definition $\pi_{*}^{\mathcal{A}(G)} X=\Psi \pi_{*}^{\mathcal{A}(\mathbb{N})}\left(\operatorname{res}_{\mathbb{N}}^{G} X\right)$, so the commutation of the left-hand square is given by the Proposition 11.8 below.

Proposition 11.8 If $X$ is a $G$-spectrum then the counit

$$
\theta_{*} \pi_{*}^{\mathcal{A}(G)}(X)=\theta_{*} \Psi \pi_{*}^{\mathcal{A}(\mathbb{N})}\left(\operatorname{res}_{\mathbb{N}}^{G} X\right) \stackrel{\cong}{\rightrightarrows} \pi_{*}^{\mathcal{A}(\mathbb{N})}\left(\operatorname{res}_{\mathbb{N}}^{G} X\right)
$$

is an isomorphism.

Remark 11.9 In essence this amounts to two classical statements about Borel cohomology (Lemma 10.1(iii) and (iv)).

Proof We consider the situation at $K \subseteq \mathbb{T}$, for a $G$-spectrum $X$, where we have the map

$$
\begin{aligned}
H^{*}(B \mathbb{T} / K) \otimes_{H^{*}\left(B W_{G}^{e} K\right)} \pi_{*}^{\mathbb{T} / K}\left(D E \mathbb{T} / K_{+} \wedge \Phi^{K} X\right)^{\mathbb{W} W_{G}^{e} K} & \\
& \longrightarrow \pi_{*}^{\mathbb{T} / K}\left(D E \mathbb{T} / K_{+} \wedge \Phi^{K} X\right) .
\end{aligned}
$$

Since $H^{*}(B \mathbb{T} / K)$ is free over $H^{*}\left(B W_{G}^{e} K\right)$, both sides commute with direct limits in $X$, so it suffices to prove this is an equivalence for finite $X$, and these may be taken to be of the form $D Y$ for a finite spectrum $Y$. Since $\Phi^{K} D Y \simeq D \Phi^{K} Y$ for finite $Y$, and since

$$
D E \mathbb{T} / K_{+} \wedge D \Phi^{K} Y \simeq D\left(E \mathbb{T} / K_{+} \wedge \Phi^{K} Y\right)
$$

we may translate this into the following statement about Borel cohomology of the $W K$-spectrum $\Phi^{K} Y$ :

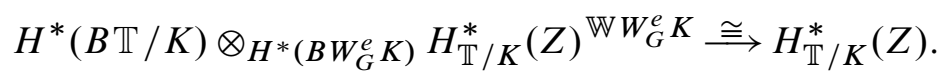

We note further that this only depends on the identity component $W_{G}^{e} K$ of $W K$, and it is sufficient to consider the special case when $Z$ is free and the suspension spectrum of a space.

The required isomorphism is then the special case $\Gamma=W_{G}^{e} K$ of the Eilenberg-Moore theorem as in Lemma 10.1(iv). This completes the proof of the proposition.

We note that Proposition 11.8 has significant consequences: only modules of the form $\theta_{*} N$ can be $\pi_{*}^{\mathcal{A}(\mathbb{N})} X$ for a $G$-spectrum $X$. 
Example 11.10 If $G=\mathrm{SO}(3)$ we have $\mathbb{N}=O(2)$ and $\mathbb{T}=\mathrm{SO}(2)$. Therefore $H^{*}(B \mathbb{T})=\mathbb{Q}[c]$ for $c$ of degree -2 with $W=O(2) / \mathrm{SO}(2)$ acting as -1 on $c$, and $H^{*}(B G)=H^{*}(B \mathbb{T})^{W}=\mathbb{Q}[d]$ where $d=c^{2}$ is of degree -4 .

We thus find that the only $\mathbb{Q}[c][W]$-modules occurring as the $\mathbb{T}$-equivariant homotopy of a free $G$-spectrum are those of the form $M=\mathbb{Q}[c] \otimes_{\mathbb{Q}[d]} N$. In particular the eigenspaces of +1 and -1 are related by

$$
M^{-}=c \cdot N=\Sigma^{-2} N=\Sigma^{-2} M^{+} .
$$

For example $\mathbb{Q}[c] /\left(c^{2}\right)=\mathbb{Q} \oplus \Sigma^{-2} \widetilde{\mathbb{Q}}$ occurs, but the dual module $\mathbb{Q} \oplus \Sigma^{2} \widetilde{\mathbb{Q}}$ does not.

Proposition 11.8 gives the beginning of our main change of groups theorem.

Corollary 11.11 If $X$ and $Y$ are $G$-spectra then

$$
\operatorname{Hom}_{\mathcal{A}(G, \text { toral })}\left(\pi_{*}^{\mathcal{A}(G)} X, \pi_{*}^{\mathcal{A}(G)} Y\right)=\operatorname{Hom}_{\mathcal{A}(\mathbb{T})}\left(\pi_{*}^{\mathcal{A}(\mathbb{T})} X, \pi_{*}^{\mathcal{A}(\mathbb{T})} Y\right)^{\mathbb{W} G} .
$$

\section{$11 E$ Coinduction}

We have just shown that $\theta_{*}$ models restriction. If the algebraic and topological categories were equivalent, it would follow that the right adjoint of $\theta_{*}$ (viz $\Psi$ ) modelled the right adjoint of restriction (viz coinduction). We show that this expected relationship does indeed hold.

Proposition 11.12 For any $\mathbb{N}$-spectrum $Y$,

$$
\pi_{*}^{\mathcal{A}(G)}\left(F_{\mathbb{N}}\left(G_{+}, Y\right)\right)=\Psi \pi_{*}^{\mathcal{A}(\mathbb{N})}(Y),
$$

so that the following diagram commutes:

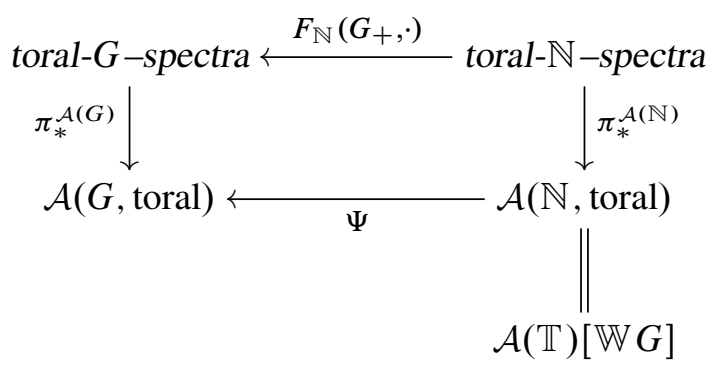

Remark 11.13 In essence this amounts to a classical statement about Borel cohomology (Corollary 10.2). 
Proof First we note there is a natural transformation. Indeed, we may apply $\Psi \pi_{*}^{\mathcal{A}(\mathbb{N})}$ to the counit

$$
\left.\operatorname{res}_{\mathbb{N}}^{G} F_{\mathbb{N}}\left(G_{+}, Y\right)\right) \longrightarrow Y
$$

to obtain a natural map

$$
\pi_{*}^{\mathcal{A}(G)}\left(F_{\mathbb{N}}\left(G_{+}, Y\right)\right)=\Psi\left(\pi_{*}^{\mathcal{A}(\mathbb{N})}\left(\operatorname{res}_{\mathbb{N}}^{G} F_{\mathbb{N}}\left(G_{+}, Y\right)\right)\right) \longrightarrow \Psi\left(\pi_{*}^{\mathcal{A}(\mathbb{N})}(Y)\right) .
$$

Both of these are cohomology theories in the toral $\mathbb{N}$-spectrum $Y$, so it suffices to show that the map is an equivalence when $Y=D \mathbb{N} / K_{+}$for $K \subseteq \mathbb{T}$. Thus we need only check that $\Psi \pi_{*}^{\mathcal{A}(\mathbb{N})}$ vanishes on the cofibre of $\mathbb{N} / K_{+} \rightarrow G / K_{+}$, which was Corollary 10.4 .

\section{An Adams spectral sequence}

We need to set up a means of calculation, so we will construct an Adams spectral sequence based on $\mathcal{A}(G$, toral $)$. We summarize the method here, referring to the appropriate sections for proofs.

\section{A Overview}

The main theorem of the paper is as follows.

Theorem 12.1 There is an Adams spectral sequence for calculating maps between toral $G$-spectra. For arbitrary rational toral $G$-spectra $X$ and $Y$ there is a strongly convergent spectral sequence

$$
E_{2}^{s, t}=\operatorname{Ext}_{\mathcal{A}(G, \text { toral })}^{s, t}\left(\pi_{*}^{\mathcal{A}(G)}(X), \pi_{*}^{\mathcal{A}(G)}(Y)\right) \Rightarrow[X, Y]_{t-s}^{G} .
$$

The $E_{2}$-page lies between the $s=0$ line and the $s=r$ line, where $r$ is the rank of $G$, so the spectral sequence collapses at the $E_{r+1}$-page.

Proof We outline the standard strategy and deal with the main points in succession.

First, Proposition 8.3 shows that the abelian category $\mathcal{A}(G$, toral $)$ has enough injectives. Accordingly, we may form an injective resolution

$$
0 \longrightarrow \pi_{*}^{\mathcal{A}(G)}(Y) \longrightarrow I_{0} \longrightarrow I_{1} \longrightarrow \cdots
$$

of $\pi_{*}^{\mathcal{A}(G)}(Y)$ in $\mathcal{A}(G$, toral $)$.

We then show that this can be realized by toral spectra. First the objects. 
Lemma 12.2 Enough injectives are realizable, that is, there are enough injectives $I$ in $\mathcal{A}\left(G\right.$, toral) for which there exist toral $G$-spectra $\mathbb{I}$ with $\pi_{*}^{\mathcal{A}(G)}(\mathbb{I})=I$.

This is proved in Section 13 .

Next we show that maps between the injectives are realizable.

Proposition 12.3 If $\mathbb{I}$ is one of the injectives constructed in the proof of Lemma 12.2, then we have an isomorphism

$$
\begin{aligned}
\pi_{*}^{\mathcal{A}(G)}:[X, \mathbb{I}]^{G} \longrightarrow & \operatorname{Hom}_{\mathcal{A}(G, \text { toral })}\left(\pi_{*}^{\mathcal{A}(G)}(X), \pi_{*}^{\mathcal{A}(G)}(\mathbb{I})\right) \\
& =\operatorname{Hom}_{\mathcal{A}(G, \text { toral })}\left(\pi_{*}^{\mathcal{A}(G)}(X), I\right) .
\end{aligned}
$$

This is proved in Section 14.

This enables us to construct an Adams tower:

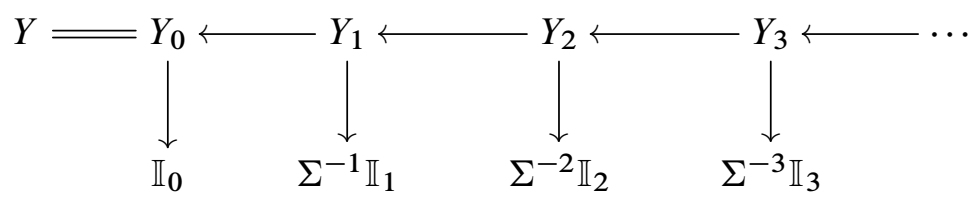

The construction starts by using Lemma 12.2 to realize $I_{0}$ by a $G$-spectrum $\mathbb{I}_{0}$ and then Proposition 12.3 to realize $\pi_{*}^{\mathcal{A}}(Y) \rightarrow I_{0}$ by a map $Y \rightarrow \mathbb{I}_{0}$. We now take $Y_{1}$ to be its fibre so that $\pi_{*}^{\mathcal{A}(G)}\left(\Sigma Y_{1}\right)=\operatorname{cok}\left(\pi_{*}^{\mathcal{A}(G)}(Y) \rightarrow I_{0}\right)$. We may now repeat, using Lemma 12.2 to realize $I_{1}$ and Proposition 12.3 to give a map $Y_{1} \rightarrow \Sigma^{-1} \mathbb{I}_{1}$ realizing the map in the algebraic resolution. Higher Adams covers are constructed by continuing this process.

This process terminates by Proposition 8.3 , which shows the category $\mathcal{A}(G$, toral) has finite injective dimension.

We deduce that the Adams tower stops at $Y_{r+1}$ with $\pi_{*}^{\mathcal{A}(G)}\left(Y_{r+1}\right)=0$. Applying $[X, \cdot]^{G}$ to the tower we obtain a spectral sequence. By Proposition 12.3 it has the stated $E_{2}$ term. The convergence statement is as follows.

Lemma 12.4 If $X$ is a toral $G$-spectrum with $\pi_{*}^{\mathcal{A}(G)}(X)=0$ then $X \simeq *$.

Proof Suppose then that $\pi_{*}^{\mathcal{A}(G)}(X)=0$, and we want to prove that $X$ is contractible. By Proposition 9.3 it suffices to show that $\pi_{*}^{\mathcal{A}(\mathbb{T})}(X)=0$. By definition, we have $\pi_{*}^{\mathcal{A}(G)}(X)=\Psi \pi_{*}^{\mathcal{A}(\mathbb{T})}(X)$, so the result follows, since by Proposition 11.8

$$
\pi_{*}^{\mathcal{A}(\mathbb{N})}(X)=\theta_{*} \Psi \pi_{*}^{\mathcal{A}(\mathbb{N})} X=\theta_{*} \pi_{*}^{\mathcal{A}(G)} X .
$$

Modulo the deferred proofs of the lemmas, this completes the proof of Theorem 12.1. 


\section{Realizing enough injectives}

In this subsection we prove Lemma 12.2 by realizing enough of the injectives described in Section 8.

\section{A Supports}

For a commutative Noetherian ring, the indecomposable injectives correspond to the prime ideals, and the injective corresponding to a prime $\wp$ is the injective hull of the residue field of $\wp$. The support of a sum of these is the collection of primes involved. The same principle applies in our context. We have notions of algebraic and geometric injectives and in both cases the support is a set of closed subgroups.

In $\mathcal{A}(\mathbb{T})$ the support is given by the maximal subgroup on which a module is nonzero. This means that the primes correspond to closed subgroups $K$, and the ring corresponding to $K$ is $H^{*}(B \mathbb{T} / K)$ with residue field $\mathbb{Q}$ and injective hull $H_{*}(B \mathbb{T} / K)$. To obtain the corresponding object of $\mathcal{A}(\mathbb{T})$, we apply the functor $f_{K}^{\mathbb{T}}$ right adjoint to evaluation at $K$.

Moving from $\mathbb{T}$ to $\mathbb{N}$, the same idea works for $\mathcal{A}(\mathbb{N}$, toral $)=\mathcal{A}(\mathbb{T})[\mathbb{W} G]$, as we saw in Section 8, provided we use the complete $\mathbb{W} G$-orbit $(K)$ rather than the singleton $K$. For $G$, the support is detected through restriction to $\mathbb{N}$.

\section{B Some idempotent spaces}

The support in the topological setting corresponds to geometric isotropy. Indecomposable injectives are realized by the simplest possible space with geometric isotropy equal to the support. We pause to catalogue some of these spaces.

The geometric isotropy

$$
\mathcal{G I}(X)=\left\{K \mid \Phi^{K} X \Varangle_{1} *\right\}
$$

consists of subgroups where the geometric fixed points are nonequivariantly essential. We further restrict to spectra where the geometric fixed points are nonequivariantly either $S^{0}$ or contractible, which we might call "locally idempotent".

We recall that a collection $\mathcal{H}$ of subgroups closed under conjugacy is called a family if it is closed under passage to subgroups, it is called a cofamily if it is closed under passage to supergroups, and it is called an interval if it contains any subgroup $K$ which lies between two elements of $\mathcal{H}$. Intervals of subgroups are precisely those collections which are the intersection of a family and a cofamily. 
Definition 13.1 If $\mathcal{H}$ is an interval of subgroups we write $\Lambda(\mathcal{H})$ for the set of subgroups of elements of $\mathcal{H}$ (which is the smallest family containing $\mathcal{H}$ ) and $V(\mathcal{H})$ for the set of supergroups of elements of $\mathcal{H}$ (which is the smallest cofamily containing $\mathcal{H}$ ) and we define

$$
E\langle\mathcal{H}\rangle:=E \Lambda(\mathcal{H})_{+} \wedge \widetilde{E}(\mathrm{All} \backslash V(\mathcal{H})) .
$$

The proof of the following lemma is immediate from the geometric fixed point Whitehead theorem.

Lemma 13.2 If $\mathcal{H}$ is an interval, and we choose a family $\mathcal{F}$ of subgroups and a cofamily $\mathcal{C}$ of subgroups so that $\mathcal{H}=\mathcal{F} \cap \mathcal{C}$ then

$$
E\langle\mathcal{H}\rangle \simeq E \mathcal{F}_{+} \wedge \tilde{E}(\operatorname{All} \backslash \mathcal{C})
$$

The space $E\langle\mathcal{H}\rangle$ is an idempotent spectrum with geometric isotropy $\mathcal{H}$, and any other locally idempotent spectrum with geometric isotropy $\mathcal{H}$ is equivalent to it.

Remark 13.3 It is worth recording the following easy observations:

(1) $\mathcal{G} \mathcal{I}(E\langle\mathcal{H}\rangle)=\mathcal{H}$.

(2) If $\mathcal{F}$ is a family then

$$
E\langle\mathcal{F}\rangle=E \mathcal{F}_{+}
$$

(3) If $\mathcal{C}$ is a cofamily then

$$
E\langle\mathcal{C}\rangle=\widetilde{E}(\text { All } \backslash \mathcal{C})
$$

(4) Given two intervals $\mathcal{H}_{1}$ and $\mathcal{H}_{2}$ we have an equivalence

$$
E\left\langle\mathcal{H}_{1}\right\rangle \wedge E\left\langle\mathcal{H}_{2}\right\rangle \simeq E\left\langle\mathcal{H}_{1} \cap \mathcal{H}_{2}\right\rangle .
$$

(5) If $K$ is a subgroup of $G$ and $\mathcal{H}$ is an interval of subgroups of $G$, we may consider the interval $\left.\mathcal{H}\right|_{K}$ of subgroups of $K$ from $\mathcal{H}$ and then

$$
\operatorname{res}_{K}^{G} E_{G}\langle\mathcal{H}\rangle=E_{K}\left\langle\left.\mathcal{H}\right|_{K}\right\rangle \text {. }
$$

\section{C Idempotent spaces from conjugacy classes}

We apply the generalities in our standard context with $G$ a compact Lie group with maximal torus $\mathbb{T}$ and $\mathbb{N}=N_{G}(\mathbb{T})$.

The spectra we are concerned with are idempotent spectra with all the geometric isotropy groups coming from a single conjugacy class in a larger group. The point of the previous subsection was to point out that in this case the geometric isotropy 
determines the object. This subsection records some immediate consequences for single conjugacy classes.

For the interval $(K)_{G}$ we consider the space

$$
E_{G}\langle K\rangle=E \Lambda_{G}(K)_{+} \wedge \tilde{E}\left(\mathrm{All} \backslash V_{G}(K)\right)
$$

where $\Lambda_{G}(K)$ is the family of subgroups $G$-subconjugate to $K$ and $V_{G}(K)$ is the cofamily of subgroups containing a $G$-conjugate of $K$. In the following, it is helpful to introduce some temporary notation. We write $P=N_{\mathbb{N}} K$ for the subgroup of $\mathbb{N}$ fixing $K$, and we suppose the $\mathbb{N}$-conjugacy class of $K$ is $\left\{K_{1}, \ldots, K_{s}\right\}$, so that $s=|\mathbb{N}: P|$.

Lemma 13.4 There is an equivalence of $\mathbb{T}$-spectra

$$
\operatorname{res}_{\mathbb{T}}^{P} E_{P}\langle K\rangle \simeq E_{\mathbb{T}}\langle K\rangle
$$

There is an equivalence of $\mathbb{N}$-spectra

$$
e_{(\mathbb{T})} \operatorname{res}_{\mathbb{N}}^{G} E_{G}\langle K\rangle \simeq E_{\mathbb{N}}\langle K\rangle \simeq \mathbb{N}_{+} \wedge_{P} E_{\mathbb{T}}\langle K\rangle,
$$

and hence an equivalence of $\mathbb{T}$-spectra

$$
\operatorname{res}_{\mathbb{T}}^{G} E_{G}\langle K\rangle \simeq \bigvee_{i=1}^{s} E_{\mathbb{T}}\left\langle K_{i}\right\rangle .
$$

Remark 13.5 The idempotent in the second statement is necessary. Consider the special case of $G=\mathrm{SO}(3)$, where $\mathbb{N}=O(2)$ and $\mathbb{T}=\mathrm{SO}(2)$. The dihedral group of order 2 in $O(2)$ is not conjugate in $O(2)$ to a subgroup of $\mathbb{T}$, but in $\mathrm{SO}(3)$ it is.

Proof The first equivalence is clear.

Two subgroups of $\mathbb{T}$ which are conjugate in $G$ are conjugate in $\mathbb{N}$ (the proof for elements in [4, IV.2.5] applies to cover noncyclic subgroups of $\mathbb{T}$ ). The geometric isotropy of $E_{G}\langle K\rangle$ is the single conjugacy class $(K)_{G}$. The part lying in $\mathbb{T}$ is the $\mathbb{N}$-conjugacy class.

There is a natural map of $\mathbb{N} \cap N_{G}(K)$-spaces $E_{\mathbb{T}}\langle K\rangle \rightarrow E_{G}\langle K\rangle$ which is $\{K\} \rightarrow(K)_{\mathbb{N}}$ on supports. Since $\mathbb{T}$ centralizes $K$ this extends to $\mathbb{N} \times_{\mathbb{N} \cap N_{G}(K)}\{K\} \cong(K)_{\mathbb{N}}$.

The interaction with coinduction is important. Note that in coinducing from $E_{\mathbb{T}}\langle K\rangle$ there are three significant stopping points: $P=N_{\mathbb{N}} K$ (since $\left.\{K\}=(K)_{\mathbb{T}}=(K)_{P}\right), \mathbb{N}$ and $G$. 
Lemma 13.6 If $K$ is a subgroup of $\mathbb{T}$ and $P=N_{\mathbb{N}} K$ then we have the following two equivalences of $P$-spectra:

$$
F_{\mathbb{T}}\left(P_{+}, E_{\mathbb{T}}\langle K\rangle\right) \simeq P / \mathbb{T}_{+} \wedge E_{P}\langle K\rangle
$$

and

$$
F_{\mathbb{T}}\left(\mathbb{N}_{+}, E_{\mathbb{T}}\langle K\rangle\right) \simeq P / \mathbb{T}_{+} \wedge E_{\mathbb{N}}\langle K\rangle
$$

Proof The first statement is a standard untwisting result.

For the second, we calculate

$$
\begin{aligned}
F_{\mathbb{T}}\left(\mathbb{N}_{+}, E_{\mathbb{T}}\langle K\rangle\right) & \simeq F_{P}\left(\mathbb{N}_{+}, F_{\mathbb{T}}\left(P_{+}, E_{\mathbb{T}}\langle K\rangle\right)\right. \\
& \simeq F_{P}\left(\mathbb{N}_{+}, P / \mathbb{T}_{+} \wedge E_{P}\langle K\rangle\right) \\
& \simeq \mathbb{N}_{+} \wedge_{P} P / \mathbb{T}_{+} \wedge E_{P}\langle K\rangle \\
& \simeq P / \mathbb{T}_{+} \wedge \mathbb{N}_{+} \wedge P E_{P}\langle K\rangle \\
& \simeq P / \mathbb{T}_{+} \wedge E_{\mathbb{N}}\langle K\rangle,
\end{aligned}
$$

where the final equivalence comes from Lemma 13.4.

Coinducing up to $G$ has little effect.

Lemma 13.7 There is an equivalence

$$
E_{G}\langle K\rangle \simeq F_{\mathbb{N}}\left(G_{+}, e_{(\mathbb{T})} E_{G}\langle K\rangle\right) \simeq F_{\mathbb{N}}\left(G_{+}, E_{\mathbb{N}}\langle K\rangle\right) .
$$

\section{D Realizing injectives}

Again we rely on [11], which shows that in $\mathcal{A}(\mathbb{T})$ the basic injective with support $K \subset \mathbb{T}$ corresponds to the space $E\langle K\rangle$. More precisely,

$$
\pi_{*}^{\mathcal{A}(\mathbb{T})}\left(E_{\mathbb{T}}\langle K\rangle\right)=f_{K}^{\mathbb{T}}\left(H_{*}\left((B \mathbb{T} / K)^{L \mathbb{T} / K}\right)\right)
$$

where $f_{K}^{\mathbb{T}}$ is right adjoint to evaluation at $K$ as before. Since we have now catalogued behaviour under change of groups in algebra and topology, we can now read off the values we require.

Corollary 13.8 The images of $E_{G}\langle K\rangle$ in $\mathcal{A}(\mathbb{N}$, toral) and $\mathcal{A}(G$, toral $)$ are given by the formulae

$$
\pi_{*}^{\mathcal{A}(\mathbb{N})} E_{G}\langle K\rangle=f_{(K)}^{\mathbb{N}}\left(H_{*}\left((B \mathbb{T} / K)^{L(\mathbb{T} / K)}\right)\right)
$$

and

$$
\pi_{*}^{\mathcal{A}(G)} E_{G}\langle K\rangle=f_{(K)}^{G}\left(H_{*}\left(\left(B W_{G}^{e} K\right)^{L W_{G}^{e} K}\right)\right),
$$

where $f_{(K)}^{\mathbb{N}}$ and $f_{(K)}^{G}$ are right adjoint to evaluation at $K$. 
Proof We have constructed $E_{G}\langle K\rangle$ so that its geometric isotropy is concentrated on $(K)_{\mathbb{N}}$, so the module is concentrated on conjugates of $K$ in $\mathbb{T}$. In view of equivariance, we need only identify the value at a single point in the orbit, and we find

$$
\begin{aligned}
\pi_{*}^{\mathcal{A}(\mathbb{N})}\left(E_{G}\langle K\rangle\right)(K) & =\pi_{*}^{\mathbb{T} / K}\left(D E \mathbb{T} / K_{+} \wedge E \mathbb{T} / K_{+}\right) \\
& =\pi_{*}^{\mathbb{T} / K}\left(E \mathbb{T} / K_{+}\right) \\
& =H_{*}\left(B \mathbb{T} / K^{L(\mathbb{T} / K)}\right) .
\end{aligned}
$$

The second statement follows from the first using Lemma 13.7, since by Lemma 10.5

$$
\begin{aligned}
H_{*}\left((B \mathbb{T} / K)^{L(\mathbb{T} / K)}\right)^{W_{G}^{e} K} & \cong H_{*}\left((B \mathbb{T} / K)^{L(\mathbb{T} / K)}\right)_{W_{G}^{e} K} \\
& \cong H_{*}\left(B W_{G}^{e} K\right)^{L\left(W_{G}^{e} K\right)} .
\end{aligned}
$$

We actually need slightly more general injectives, so that we can embed all representations of $W_{G}^{d}(K)$. Of course there are many possible choices. We could start from $E_{\mathbb{N}}\langle K\rangle$ and coinduce, but it turns out that the proof is slightly streamlined by starting from $E_{\mathbb{T}}\langle K\rangle$. We give the calculations for both by way of comparison.

Corollary 13.9 The images of $\mathbb{N} / \mathbb{T}_{+} \wedge E_{\mathbb{N}}\langle K\rangle$ in $\mathcal{A}(\mathbb{N}$, toral) and its coinduced spectrum $F_{\mathbb{T}}\left(G_{+}, E_{\mathbb{N}}\langle K\rangle\right)$ in $\mathcal{A}(G$, toral $)$ are given by the formulae

$$
\pi_{*}^{\mathcal{A}(\mathbb{N})}\left(\mathbb{N} / \mathbb{T}_{+} \wedge E_{\mathbb{N}}\langle K\rangle\right)=f_{(K)}^{\mathbb{N}}\left(\mathbb{Q}[\mathbb{W} G] \otimes H_{*}\left((B \mathbb{T} / K)^{L(\mathbb{T} / K)}\right)\right)
$$

and

$$
\begin{aligned}
\pi_{*}^{\mathcal{A}(G)}\left(F_{\mathbb{T}}\left(G_{+}, E_{\mathbb{N}}\langle K\rangle\right)\right) & =\Psi f_{(K)}^{\mathbb{N}}\left(\mathbb{Q}[\mathbb{W} G] \otimes H_{*}\left((B \mathbb{T} / K)^{L(\mathbb{T} / K)}\right)\right) \\
& =f_{(K)}^{G}\left(\mathbb{Q}\left[\mathbb{W} G / W G_{K}^{e}\right] \otimes H_{*}\left((B \mathbb{T} / K)^{L \mathbb{T} / K}\right)\right),
\end{aligned}
$$

where $f_{(K)}^{\mathbb{N}}$ and $f_{(K)}^{G}$ are right adjoints to evaluation at $K$.

Proof The statement for $\mathbb{N}$ follows easily from the previous corollary, recalling from Section $8 \mathrm{~A}$ that modules over $(K)_{\mathbb{N}}$ are determined from their value over $K$ by conjugation.

The statement for $G$ follows since $\Psi$ models coinduction as in Proposition 11.12.

We note that if $N$ is an $H^{*}(B \mathbb{T} / K)\left[\mathbb{W} G_{K}\right]$-module, there is a natural transformation

$$
f_{(K)}^{G}(\Psi N)=\Psi f_{(K)}^{\mathbb{N}}\left(\theta_{*} \Psi N\right) \longrightarrow \Psi f_{(K)}^{\mathbb{N}}(N),
$$


and when evaluated at $K$ the comparison is the identity

$$
\Psi \theta_{*} \Psi N \longrightarrow \Psi N \text {. }
$$

The values that we will actually use in the proofs are as follows.

Corollary 13.10 The images of the coinduction of $E_{\mathbb{T}}\langle K\rangle$ to $\mathbb{N}$-spectra and to $G$-spectra in the algebraic categories are given by the formulae

$$
\pi_{*}^{\mathcal{A}(\mathbb{N})}\left(F_{\mathbb{T}}\left(\mathbb{N}_{+}, E_{\mathbb{T}}\langle K\rangle\right)\right)=f_{(K)}^{\mathbb{N}}\left(\mathbb{Q}\left[(\mathbb{W} G)_{K}\right] \otimes H_{*}\left((B \mathbb{T} / K)^{L(\mathbb{T} / K)}\right)\right)
$$

and

$$
\begin{aligned}
\pi_{*}^{\mathcal{A}(G)}\left(F_{\mathbb{T}}\left(G_{+}, E_{\mathbb{T}}\langle K\rangle\right)\right) & =\Psi f_{(K)}^{\mathbb{N}}\left(\mathbb{Q}\left[(\mathbb{W} G)_{K}\right] \otimes H_{*}\left((B \mathbb{T} / K)^{L(\mathbb{T} / K)}\right)\right) \\
& =f_{(K)}^{G}\left(\mathbb{Q}\left[W_{G}^{d} K\right] \otimes H_{*}\left((B \mathbb{T} / K)^{L \mathbb{T} / K}\right)\right),
\end{aligned}
$$

where $f_{(K)}^{\mathbb{N}}$ and $f_{(K)}^{G}$ are right adjoints to evaluation at $K$.

Proof If we note that $(\mathbb{W} G)_{K}=N_{\mathbb{N}} K / \mathbb{T}=P / \mathbb{T}$, then the first statement follows from Lemma 13.6.

The second statement follows as in the proof of Corollary 13.9.

\section{Maps into injectives}

In this section we give control over maps to realizable injectives through proving Proposition 12.3. Since this is where we get control over the maps in our category, it is perhaps not surprising that it is the most delicate part of the argument.

Proposition 14.1 If $\mathbb{I}$ is a $G$-spectrum realizing one of the injectives $I$ constructed in the proof of Lemma 12.2, then we have an isomorphism

$$
\begin{aligned}
\pi_{*}^{\mathcal{A}(G)}:[X, \mathbb{I}]^{G} \longrightarrow & \operatorname{Hom}_{\mathcal{A}(G, \text { toral })}\left(\pi_{*}^{\mathcal{A}(G)}(X), \pi_{*}^{\mathcal{A}(G)}(\mathbb{I})\right) \\
& =\operatorname{Hom}_{\mathcal{A}(G, \text { toral })}\left(\pi_{*}^{\mathcal{A}(G)}(X), I\right) .
\end{aligned}
$$

Proof Since $I$ is injective, both sides are cohomology theories of $X$, and it suffices to prove the result for $X=G / K_{+}$where $K$ is a subgroup of $\mathbb{T}$. In fact we will prove it more generally for $X=G_{+} \wedge_{\mathbb{T}} A$ for some finite $\mathbb{T}$-spectrum $A$. We consider the 
diagram:

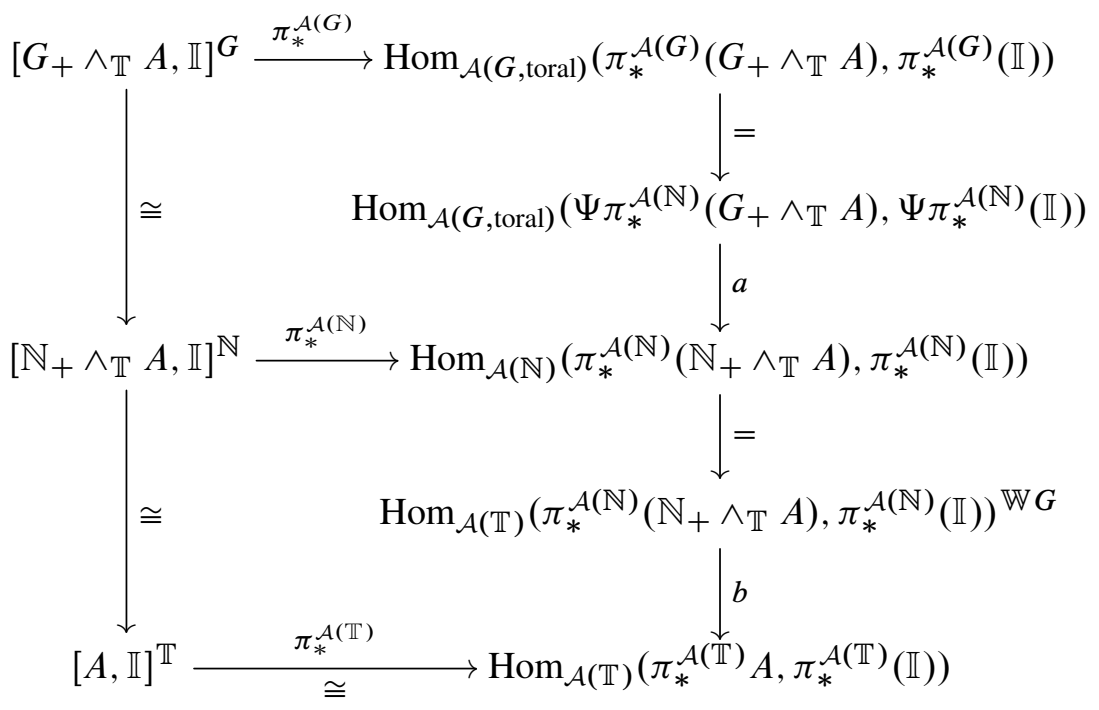

The bottom horizontal is an isomorphism from the $\mathbb{T}$-equivariant Adams spectral sequence of [11], since $\pi_{*}^{\mathcal{A}(\mathbb{T})}(\mathbb{I})$ is injective. The two left-hand vertical isomorphisms come from the induction-restriction adjunction. The two right-hand vertical isomorphisms are definitions.

It therefore remains to describe the maps $a$ and $b$ so that the diagram commutes and to show that $a$ and $b$ are isomorphisms.

We will deal with $b$ first, because it is straightforward. Since $\pi_{*}^{\mathcal{A}(\mathbb{N})}=\pi_{*}^{\mathcal{A}(\mathbb{T})}$ if we ignore the $\mathbb{W} G$-action, we may take $b$ to be induced by the $\mathbb{T}$-map $\beta: A \rightarrow \mathbb{N} \wedge_{\mathbb{T}} A$. The diagram commutes, since by definition the left-hand vertical factors through the forgetful map

$$
\left[\mathbb{N}_{+} \wedge_{\mathbb{T}} A, \mathbb{I}\right]^{\mathbb{N}} \longrightarrow\left[\mathbb{N}_{+} \wedge_{\mathbb{T}} A, \mathbb{I}\right]^{\mathbb{T}}
$$

The fact that $b$ is an isomorphism follows from a lemma.

Lemma 14.2 The map $\beta$ induces an isomorphism

$$
\pi_{*}^{\mathcal{A}(\mathbb{N})}\left(\mathbb{N}_{+} \wedge_{\mathbb{T}} A\right)=\mathbb{W} G \otimes \pi_{*}^{\mathcal{A}(\mathbb{T})}(A),
$$

where the functor on the right is the induction functor left adjoint to restriction. 
For the map $a$ we use the diagram:

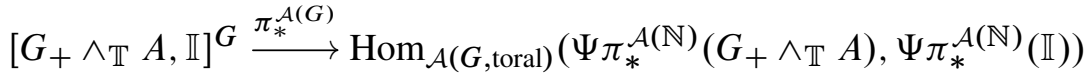

$$
\begin{aligned}
& \cong \theta_{*} \\
& {\left[G_{+} \wedge_{\mathbb{T}} A, \mathbb{I}\right]^{\mathbb{N}} \stackrel{\pi_{*}^{\mathcal{A}(\mathbb{N})}}{\longrightarrow} \operatorname{Hom}_{\mathcal{A}(\mathbb{T})}\left(\pi_{*}^{\mathcal{A}(\mathbb{N})}\left(G_{+} \wedge_{\mathbb{T}} A\right), \pi_{*}^{\mathcal{A}(\mathbb{N})}(\mathbb{I})\right)^{\mathbb{W} G}}
\end{aligned}
$$

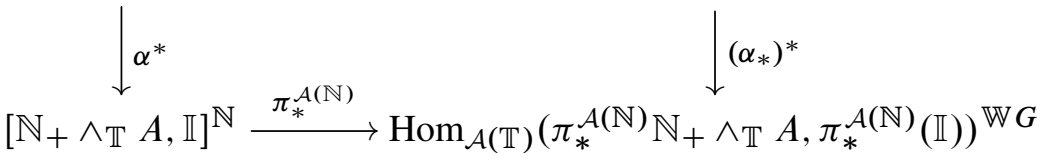

We have used the fact that the counit is an isomorphism on restrictions from $G$ (Proposition 11.8) to identify the codomain of $\theta_{*}$ and to see it is an isomorphism. In short, $a$ comes from the map

$$
\alpha_{*}: \pi_{*}^{\mathcal{A}(\mathbb{N})}\left(\mathbb{N}_{+} \wedge_{\mathbb{T}} A\right) \longrightarrow \pi_{*}^{\mathcal{A}(\mathbb{N})}\left(G_{+} \wedge_{\mathbb{T}} A\right)
$$

induced by the $\mathbb{N}-$ map $\alpha: \mathbb{N}_{+} \wedge_{\mathbb{T}} A \rightarrow G_{+} \wedge_{\mathbb{T}} A$.

We will show that $\left(\alpha_{*}\right)^{*}$ is an isomorphism, but we pause to observe that this is fairly subtle, since the map $\alpha_{*}$ itself is usually not an isomorphism.

Example 14.3 Consider the special case $\mathbb{I}=E G_{+}$. We have:

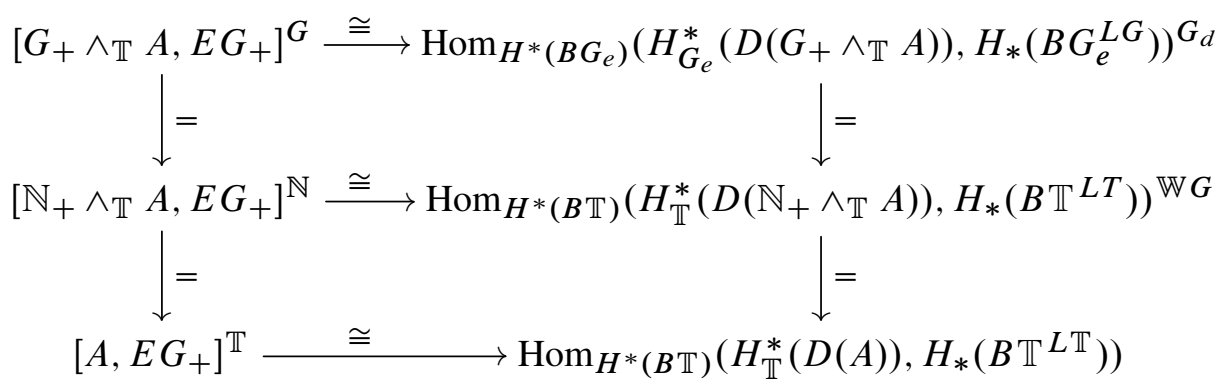

The reader may find it instructive to think how the suspensions match up.

More specifically still, we may take $G=\mathrm{SO}(3), \mathbb{N}=O(2)$ and $\mathbb{T}=\mathrm{SO}(2)$, with $A=S^{0}$. Of course $H_{\mathbb{T}}^{*}(D A)=H^{*} B \mathbb{T}$ so that we see from the bottom right that the value is $\mathbb{Q}$ in each positive degree and 0 elsewhere. At the top left, we use the fact that $H_{\mathbb{T}}^{*}\left(D G / \mathbb{T}_{+}\right)$is a copy of $\mathbb{Q}$ in codegree -2 and a copy of $\mathbb{Q} \mathbb{W} G$ in even codegrees greater than or equal to 0 , and its ring of $\mathbb{W} G$-invariants $H_{G_{e}}^{*}\left(D G / \mathbb{T}_{+}\right)$is a free $H^{*}\left(B G_{e}\right)$-module on generators of cohomological degrees 0 and -2 .

To make further progress, it is convenient to make a specific choice for $\mathbb{I}$. Indeed, since $\pi_{*}^{\mathcal{A}(\mathbb{N})}\left(G_{+} \wedge_{\mathbb{T}} A\right)$ and $\pi_{*}^{\mathcal{A}(\mathbb{N})}\left(\mathbb{N}_{+} \wedge_{\mathbb{T}} A\right)$ are small, it suffices to deal with 
the case $\mathbb{I}=F_{\mathbb{T}}\left(G_{+}, E_{\mathbb{T}}\langle K\rangle\right)$ for some $K$. For any finite $\mathbb{N}$-spectrum $B$ we have $\Phi^{K} D B=D \Phi^{K} B$ and Corollary 13.10 gives the value $\pi_{*}^{\mathcal{A}(\mathbb{N})}(\mathbb{I})$. Abbreviating $\mathcal{A}(\mathbb{N}$, toral) to $\mathcal{A}(\mathbb{N})$, we may calculate

$$
\begin{aligned}
\operatorname{Hom}_{\mathcal{A}(\mathbb{N})}\left(\pi_{*}^{\mathcal{A}(\mathbb{N})}(B), \pi_{*}^{\mathcal{A}(\mathbb{N})}\left(F_{\mathbb{T}}\left(G_{+}, E_{\mathbb{T}}\langle K\rangle\right)\right)\right) \\
\cong \operatorname{Hom}_{\mathcal{A}(\mathbb{N})}\left(\pi_{*}^{\mathcal{A}(\mathbb{N})}(B), \theta_{*} \Psi \pi_{*}^{\mathcal{A}(\mathbb{N})}\left(F_{\mathbb{T}}\left(\mathbb{N}_{+}, E_{\mathbb{T}}\langle K\rangle\right)\right)\right) \\
\cong \operatorname{Hom}_{\mathcal{A}(\mathbb{N})}\left(\pi_{*}^{\mathcal{A}(\mathbb{N})}(B), f_{K}^{\mathbb{N}}\left(\theta_{*} \Psi H_{*}\left(B \mathbb{T} / K^{L \mathbb{T} / K}\right)\left[(\mathbb{W} G)_{K}\right]\right)\right) \\
\left.\cong \operatorname{Hom}_{H^{*}(B \mathbb{T} / K)}\left(H_{\mathbb{T} / K}^{*}\left(D \Phi^{K} B\right), \theta_{*} \Psi H_{*}\left(B \mathbb{T} / K^{L \mathbb{T} / K}\right)\left[(\mathbb{W} G)_{K}\right]\right)\right)^{(\mathbb{W} G)_{K}} \\
\left.\cong \operatorname{Hom}_{H^{*}(B \mathbb{T} / K)}\left(H_{\mathbb{T} / K}^{*}\left(D \Phi^{K} B\right), \theta_{*} H_{*}\left(B \mathbb{T} / K^{L \mathbb{T} / K}\right)\left[W_{G}^{d} K\right]\right)\right)^{(\mathbb{W} G)_{K}} \\
\left.\cong \operatorname{Hom}_{H^{*}(B \mathbb{T} / K)}\left(H_{\mathbb{T} / K}^{*}\left(D \Phi^{K} B\right), \theta_{*} H_{*}\left(B \mathbb{T} / K^{L \mathbb{T} / K}\right)\right)\right)^{(\mathbb{W} G)_{K}^{e}} \\
\left.\cong \operatorname{Hom}_{H^{*}(B \mathbb{T} / K)}\left(H_{\mathbb{T} / K}^{*}\left(D \Phi^{K} B^{L \mathbb{T} / K}\right), \theta_{*} H_{*}(B \mathbb{T} / K)\right)\right)^{\mathbb{W} W_{G}^{e} K}
\end{aligned}
$$

As an $H^{*}\left(B W_{G}^{e} K\right)$-module $H_{*}(B \mathbb{T} / K)$ is a sum of copies of $H_{*}\left(B W_{G}^{e} K\right)$, and hence as an $H^{*}(B \mathbb{T} / K)$-module $\theta_{*} H_{*}(B \mathbb{T} / K)$ is a sum of copies of $H_{*}(B \mathbb{T} / K)$. The above functor is thus a sum of copies of

$\left.\operatorname{Hom}_{H^{*}(B \mathbb{T} / K)}\left(H_{\mathbb{T} / K}^{*}\left(D \Phi^{K} B^{L \mathbb{T} / K}\right), H_{*}(B \mathbb{T} / K)\right)\right)^{\mathbb{W} W_{G}^{e} K}$

$$
\left.\left.\cong\left[H_{*}^{\mathbb{T} / K}\left(D \Phi^{K} B^{L \mathbb{T} / K}\right)\right)\right]^{\mathbb{W} W_{G}^{e} K} \cong H_{*}^{W_{G}^{e} K}\left(D \Phi^{K} B^{L W_{G}^{e} K}\right)\right)
$$

where the final isomorphism is from Lemma 10.5 .

It suffices to show that $\alpha$ induces an isomorphism of this functor of $B$, or equivalently that the functor vanishes on

$$
Q(A)=\operatorname{cofibre}\left(\mathbb{N}_{+} \wedge_{\mathbb{T}} A \stackrel{\alpha}{\longrightarrow} G_{+} \wedge_{\mathbb{T}} A\right) .
$$

Now the following groups vanish together:

$$
H_{*}^{W_{G}^{e} K}\left(D \Phi^{K} Q(A)^{L W_{G}^{e} K}\right), \quad H_{W_{G}^{e} K}^{*}\left(D \Phi^{K} Q(A)^{\left.L W_{G}^{e} K\right)}\right), \quad H_{W_{G}^{e} K}^{*}\left(\Phi^{K} Q(A)\right) .
$$

The first two are vector space duals, and the last two vanish together by the standard observation about ring spectra recalled in Section 10D. The result follows from Corollary 10.4 .

\section{Essential surjectivity}

We want to show that the functors $\pi_{*}^{\mathcal{A}}$ are essentially surjective, so that our modelling categories are no bigger than necessary.

Lemma 15.1 Every object of $\mathcal{A}(G$, toral $)$ is realizable by a toral $G$-spectrum. 
Proof We may use the ingredients of the proof of the Adams spectral sequence. Suppose then that $M$ is a module in $\mathcal{A}(G$, toral). By Proposition 8.3, this has an injective resolution

$$
0 \longrightarrow M \longrightarrow I_{0} \longrightarrow \cdots \longrightarrow I_{r} \longrightarrow 0 .
$$

We now set about constructing a toral $G$-spectrum $Y$ with $\pi_{*}^{\mathcal{A}(G)} Y=M$. When $Y$ is constructed, we will in retrospect see that we have found the dual Adams tower $\left\{Y^{s}\right\}$ where this is related to the Adams tower by cofibre sequences $Y_{S} \rightarrow Y \rightarrow Y^{s}$.

In any case, we construct a tower:

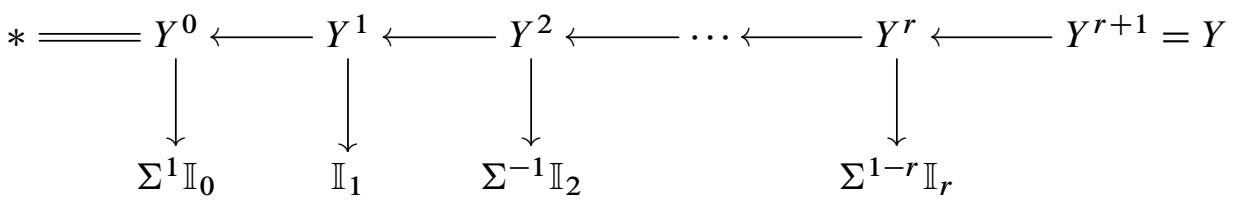

For each $s$, the $G$-spectrum $\mathbb{I}_{S}$ is a realization of $I_{S}$, which exists by Lemma 12.2. We build the tower recursively, starting with $Y^{0}=*$ and $Y^{1}=\mathbb{I}_{0}$. Supposing we have constructed the tower up to $Y^{s}$, we find an exact sequence

$$
0 \longrightarrow \Sigma^{1-s} C_{s+1} \longrightarrow \pi_{*}^{\mathcal{A}(G)} Y_{s} \longrightarrow M \longrightarrow 0
$$

in $\mathcal{A}(G$, toral $)$, where $C_{s+1}=\operatorname{im}\left(I_{s} \rightarrow I_{s+1}\right)$. Since $I_{s+1}$ is injective, we may extend the map $C_{S+1} \rightarrow I_{S+1}$ over $\pi_{*}^{\mathcal{A}(G)}\left(Y_{S}\right)$ and then by Proposition 12.3 we may realize this by a map $Y_{s} \rightarrow \Sigma^{1-s} \mathbb{I}_{s}$. We then take $Y^{s+1}$ to be the fibre, completing the step. Since $C_{r+1}=0$, the process finishes in $r$ steps with $Y=Y^{r+1}$ having $\pi_{*}^{\mathcal{A}(G)}(Y)=M$ as required.

Remark 15.2 For the special case $G=\mathbb{N}$, one may work more directly from the case of a torus.

\section{Change of groups}

We now suppose given a group $G$ and a subgroup $H$, and we choose maximal tori $S$ of $G$ and $T$ of $H$ with $S \supseteq T$. We note that it does not follow that there is a containment of normalizers of maximal tori.

Writing $i: H \rightarrow G$ for the inclusion map, the restriction map $i^{*}$ from $G$-spectra to $H$-spectra has left adjoint the induced spectrum $i_{*} Y=G_{+} \wedge_{H} Y$ and right adjoint 
$i_{!} Y=F_{H}\left(G_{+}, Y\right)$ from $G$-spectra to $H$-spectra. Applying idempotents these give functors on toral spectra:

$$
G-\text { spectra } \underset{i_{*}}{\longleftarrow} i^{*} \longrightarrow \text {-spectra } .
$$

It is the purpose of this section to describe the algebraic counterparts. If the ranks of the groups differ then there is only a good story at the level of derived functors. The exposition will deal with the general case, and simply note that if the ranks are equal then the effect of using derived functors is nugatory.

The case of a torus is considerably simpler, and since we will also reduce the general case to that of the torus, we will deal with tori first in the next subsection. For the equal rank case the content is vacuous, so readers interested only in equal rank can skip Section 16A

\section{A Tori}

In this section we consider the case when $G=S$ and $H=T$ are tori. We let $j: T \rightarrow S$ denote the inclusion and $\lambda=j^{*}: H^{*}(B S) \rightarrow H^{*}(B T)$. To prove the assertion requires working with the specific Quillen equivalences used in [15], so we will not prove it here. On the other hand, special cases can be seen: free spectra, and homologically simple objects.

Conjecture 16.1 Given an inclusion $j: T \rightarrow S$ of tori, the change of groups functors

$$
S \text {-spectra } \underset{j !}{\longleftarrow j_{*} j_{j} \leftrightarrows} T \text {-spectra }
$$

are modelled at the derived level by the functors

$$
\mathcal{A}(S) \underset{\lambda^{*}}{\frac{\lambda_{*} !}{\longleftarrow}} \mathcal{A}(T) .
$$

For an object $M$ of $\mathcal{A}(S)$, the object $\lambda_{*} M$ is defined on subgroups $L \subseteq T$ by

$$
\left(\lambda_{*} M\right)(L)=H^{*}(B T / L) \otimes_{H^{*}(B S / L)} M(L),
$$

where the tensor product is derived. For an object $N$ of $\mathcal{A}(T)$, the objects $\lambda^{*} M$ and $\lambda ! M$ are defined on subgroups $K \subseteq S$ by

$$
\left(\lambda^{*} N\right)(K)= \begin{cases}N(K) & \text { if } K \subseteq T, \\ 0 & \text { otherwise }\end{cases}
$$


and

$$
\left(\lambda^{!} N\right)(K)= \begin{cases}\Sigma^{L S / L T} N(K) & \text { if } K \subseteq T \\ 0 & \text { otherwise }\end{cases}
$$

\section{B General case}

We now return to the general case when $S$ is the maximal torus of $G$ and $T$ is the maximal torus of $H$. We write $i: H \rightarrow G$ and $j: T \rightarrow S$ for the inclusions with induced maps

$$
\theta=i^{*}: H^{*}(B G) \longrightarrow H^{*}(B H)
$$

and

$$
\lambda=j^{*}: H^{*}(B S) \longrightarrow H^{*}(B T) .
$$

We will state the proposition in the equal rank case (ie when $S=T$ ), but we have stated it so that it will hold at the derived level in general provided Conjecture 16.1 holds.

Proposition 16.2 If $G$ and $H$ have the same rank, then the change of groups functors

$$
G-\text { spectra } \underset{i_{*}}{\longleftarrow} i_{i !}^{\longleftarrow} H-\text { spectra }
$$

are modelled by the functors

$$
\mathcal{A}(G, \text { toral }) \underset{\theta^{!}}{\longleftarrow} \theta_{\theta^{*}}^{\longleftarrow} \mathcal{A}(H, \text { toral }) .
$$

For an object $M$ of $\mathcal{A}(G$, toral $), \theta_{*} M$ is defined on subgroups $L \subseteq T$ by

$$
\left(\theta_{*} M\right)(L)=\left[H^{*}(B T / L) \otimes_{H^{*}\left(B W_{G}^{e}(L)\right)} M(L)\right]^{\mathbb{W} W_{H}^{e}(L)} .
$$

For an object $N$ of $\mathcal{A}(H$, toral $), \theta^{*} M$ and $\theta^{!} M$ are defined on subgroups $K \subseteq S$ by

$$
\left(\lambda^{*} N\right)(K)= \begin{cases}{\left[H^{*}(B T / K) \otimes_{H^{*}\left(B W_{H}^{e}(K)\right)} N(K)\right]^{\mathbb{W} W_{G}^{e}(K)}} & \text { if } K \subseteq T, \\ 0 & \text { otherwise }\end{cases}
$$

and

$$
(\lambda ! N)(K)= \begin{cases}{\left[\Sigma^{L S / L T} H^{*}(B T / K) \otimes_{H^{*}\left(B W_{H}^{e}(K)\right)} N(K)\right]^{\mathbb{W} W_{G}^{e}(K)}} & \text { if } K \subseteq T, \\ 0 & \text { otherwise. }\end{cases}
$$


Remark 16.3 It is worth making explicit a couple of special cases. First note that if $H=\mathbb{N} G$ we recover part of Proposition 11.7, and similarly, if $H=\mathbb{T} G$.

The statement should hold at the derived level even when $G$ and $H$ are of different rank. If so, when $G$ and $H$ are both tori we recover Conjecture 16.1.

Proof In view of toral detection and the fact that $\Psi \theta_{*}=1$ by Proposition 5.9, we can deduce the general case from the torus case. In other words, writing $V=\mathbb{W} G$ and $W=\mathbb{W} H$, and with notation given in the diagram

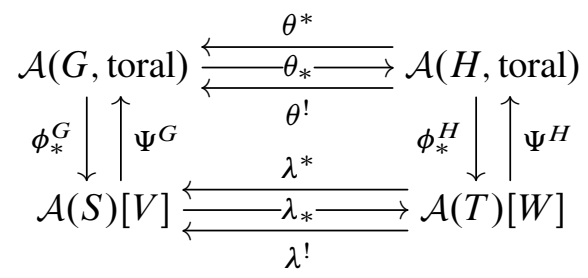

we have $\theta^{*}=\Psi^{G} \lambda^{*} \phi_{*}^{H}, \theta_{*}=\Psi^{H} \lambda_{*} \phi_{*}^{G}$ and $\theta^{!}=\Psi^{G} \lambda^{!} \phi_{*}^{H}$. The formulae are now easily verified.

\section{References}

[1] D Barnes, Classifying dihedral O(2)-equivariant spectra, preprint (2008) arXiv

[2] D Barnes, Rational equivariant spectra, $\mathrm{PhD}$ thesis (2008) arXiv

[3] D Barnes, Rational O(2)-equivariant spectra, preprint (2012) arXiv

[4] T Bröcker, T tom Dieck, Representations of compact Lie groups, Graduate Texts in Mathematics 98, Springer, New York (1985) MR

[5] T tom Dieck, The Burnside ring of a compact Lie group, I, Math. Ann. 215 (1975) 235-250 MR

[6] J P C Greenlees, Rational Mackey functors for compact Lie groups, I, Proc. London Math. Soc. 76 (1998) 549-578 MR

[7] J P C Greenlees, Rational O(2)-equivariant cohomology theories, from: "Stable and unstable homotopy", (W G Dwyer, S Halperin, R Kane, S O Kochman, M E Mahowald, P S Selick, editors), Fields Inst. Commun. 19, Amer. Math. Soc., Providence, RI (1998) 103-110 MR

[8] J P C Greenlees, Rational $S^{1}$-equivariant stable homotopy theory, Mem. Amer. Math. Soc. 661, Amer. Math. Soc., Providence, RI (1999) MR

[9] J P C Greenlees, Rational SO(3)-equivariant cohomology theories, from: "Homotopy methods in algebraic topology", (J P C Greenlees, R R Bruner, N Kuhn, editors), Contemp. Math. 271, Amer. Math. Soc., Providence, RI (2001) 99-125 MR 
[10] J P C Greenlees, Triangulated categories of rational equivariant cohomology theories, Oberwolfach Rep. 3 (2006) 480-488

[11] J P C Greenlees, Rational torus-equivariant stable homotopy, I: Calculating groups of stable maps, J. Pure Appl. Algebra 212 (2008) 72-98 MR

[12] J P C Greenlees, Rational torus-equivariant stable homotopy, II: Algebra of the standard model, J. Pure Appl. Algebra 216 (2012) 2141-2158 MR

[13] J P C Greenlees, Algebraic models of induction and coinduction, preprint (2014) arXiv

[14] J P C Greenlees, Rational torus-equivariant stable homotopy, III: Comparison of models, J. Pure Appl. Algebra 220 (2016) 3573-3609 MR

[15] J P C Greenlees, B Shipley, An algebraic model for rational torus-equivariant spectra, preprint (2011) arXiv

[16] J P C Greenlees, B Shipley, An algebraic model for free rational $G$-spectra, Bull. Lond. Math. Soc. 46 (2014) 133-142 MR

[17] M Kedziorek, Algebraic models for rational G-spectra, $\mathrm{PhD}$ thesis (2014) Available at http://etheses.whiterose.ac.uk/7699/

[18] L Solomon, Invariants of finite reflection groups, Nagoya Math. J. 22 (1963) 57-64 MR

School of Mathematics and Statistics, University of Sheffield Hicks Building, Sheffield S3 7RH, United Kingdom

j.greenlees@sheffield.ac.uk

Received: 15 January 2015 Revised: 29 October 2015 
